\title{
Analyse d'un ensemble de terre sigillée
}

Wim De Clerc, Johan Deschieter, Brenda Dickinson, Joanna Bird

\section{Citer ce document / Cite this document :}

De Clerc Wim, Deschieter Johan, Dickinson Brenda, Bird Joanna. Analyse d'un ensemble de terre sigillée. In: Revue du Nord, tome 91, n³33,1999. Archéologie de la Picardie et du Nord de la France. pp. 75-105;

doi : https://doi.org/10.3406/rnord.1999.2963

https://www.persee.fr/doc/rnord_0035-2624_1999_num_91_333_2963

Fichier pdf généré le 09/04/2018 


\begin{abstract}
Wim de Clerc and Johan Deschieter, Prospections in Steene-Pitgam : analysis of a set of sigiliated earthenware.

Over the past few years, the amateur archaelogist $\mathrm{Ph}$. Ducrocq systematically prospected the surroundings of the communes of Steen-Pitgam. The eleven " dumping grounds » of archaeological artefacts that he located belong to an extensive Roman salt extraction site. Among the pottery material, sigiliated earthenware occupies a significant position. The typological, stylistic and quantitative analysis of this pottery brings chronological precisions regarding the Gallo-Roman occupation in Steene-Pitgam, and testifies of a development of the site in the Ilnd century. The set of terra sigillata remains stretches from the Nero period to the middle of the IIIrd century. From the economic point of view one can assert that this site was supplied by the workshops of the south, the centre and the east of Gaul. The analysis of the Steene-Pitgam terra sigillata earthenware allows to add new elements to the study of this pottery in northern France and to tackle at the same time some general issues. Nevertheless, it remains obvious that results gathered from surface material must be confronted with well confined contexts from the site and with other regional sites.
\end{abstract}

\title{
Résumé
}

Au cours de ces dernières années, l'archéologue amateur Ph. Ducrocq a systématiquement prospecté les environs des communes de Steene-Pitgam. Les onze " dépotoirs " d'objets archéologiques localisés font partie d'un site salin romain étendu. Dans le matériel céramique, la terre sigillée occupe une place significative. L'examen typologique, stylistique et quantitatif de cette céramique apporte des précisions chronologiques relatives à l'occupation gallo-romaine à Steene-Pitgam, révélant un essor du site dans le lle siècle. L'ensemble de sigillée s'étend de la période Néron au milieu Ille siècle. Sur le plan économique on peut démontrer que le site a été approvisionné par les ateliers du sud, du centre et de l'est de la Gaule. L'analyse de la terre sigillée de Steene-Pitgam permet d'ajouter de nouveaux éléments à l'étude de cette céramique dans le nord de la France et en même temps d'aborder quelques problèmes généraux. Néanmoins, il est évident que les résultats obtenus à partir d'un matériel de surface doivent être comparés à des contextes bien clos du site même et d'autres sites régionaux.

Wim de Clerc et Johan Deschieter, Prospectie in Steene-Pitgam : analyse van de terra sigillata.

De laatste jaren heeft een amateur archeoloog, Ph. Ducrocq, systematisch de gemeente SteenePitgam en omgeving onderzocht. Hij heeft zo elf vindplaatsen van archeologisch materiaal in een uitgebreide romeinse zoutwinning aangetroffen. De keramiek bevat veel terra sigillata. Het typologisch, stilistisch en kwantitatief onderzoek levert stof op voor de chronologie van de gallo-romeinse aanwezigheid op de site, met een piek in de tweede eeuw. Het sigillatamateriaal dateert van de tijd van Nero tot het midden van de derde eeuw en is afkom- stig uit areliers in Zuid-, Midden- en Oostgallië. De analyse van dit materiaal voegt nieuwe elementen toe aan de studie van deze keramiekvorm in Noordfrankrijk en zelfs in een meer algemene context. Maar het resultaat van oppervlaktevondsten alleen moet vergeleken worden met dat van duidelijk omlijnde sites ter plaatse en in de streek.

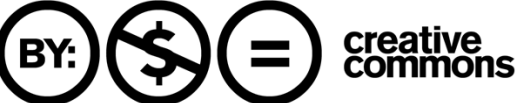




\section{Prospections à Steene-Pitgam : analyse d'un ensemble de terre sigillée}

\section{INTRODUCTION'}

\section{Le site et son contexte régional.}

Le matériel céramique que nous proposons dans cette contribution a été récupéré lors de prospections de surface sur le site salin romain de Steene-Pitgam (Nord). Durant trois années, Philippe Ducrocq (Bailleul), archéologue amateur qui a découvert le site, y a ramassé une quantité considérable d'objets archéologiques romains. Le site (fig. 1) se trouve au bord de la Colme, à la liaison entre la plaine maritime flamande et la Flandre continentale. Le sous-sol y est composé d'une argile sableuse surmontée par un limon argilo-sableux sur les terrains plus élevés. A l'époque romaine, ce site faisait partie de la région occidentale de la cité des Ménapiens. Il était relié au caput civitatis, Castellum Menapiorum (Cassel), par une voie que jalonnent des sites d'occupation gallo- romaine, ainsi sur les communes de Zégers-Cappel, Esquelbecq (La Cloche), Ledringhem et Zermezeele. La voie est désignée sous le nom de Steen Straete (Chemin des pierres) et correspond plus ou moins à l'axe de la D52 actuelle. Le tracé nord-ouest de cette voie ancienne depuis Steene-Pitgam reste difficile à suivre à cause des dépôts marins de l'assise Dunkerque II et des transgressions postérieures. Néanmoins, il est probable que pendant le HautEmpire un réseau routier reliait la Flandre continentale aux sites portuaires et aux salines supposées dans la plaine maritime.

Pour le moment, l'occupation gallo-romaine autour de Cassel et à l'est de l'estuaire de l'Aa est loin d'être bien connue. Cassel même, chef lieu de la cité des Ménapiens, ayant sans doute fait l'objet de différentes occupations militaires et civiles autant précoces que

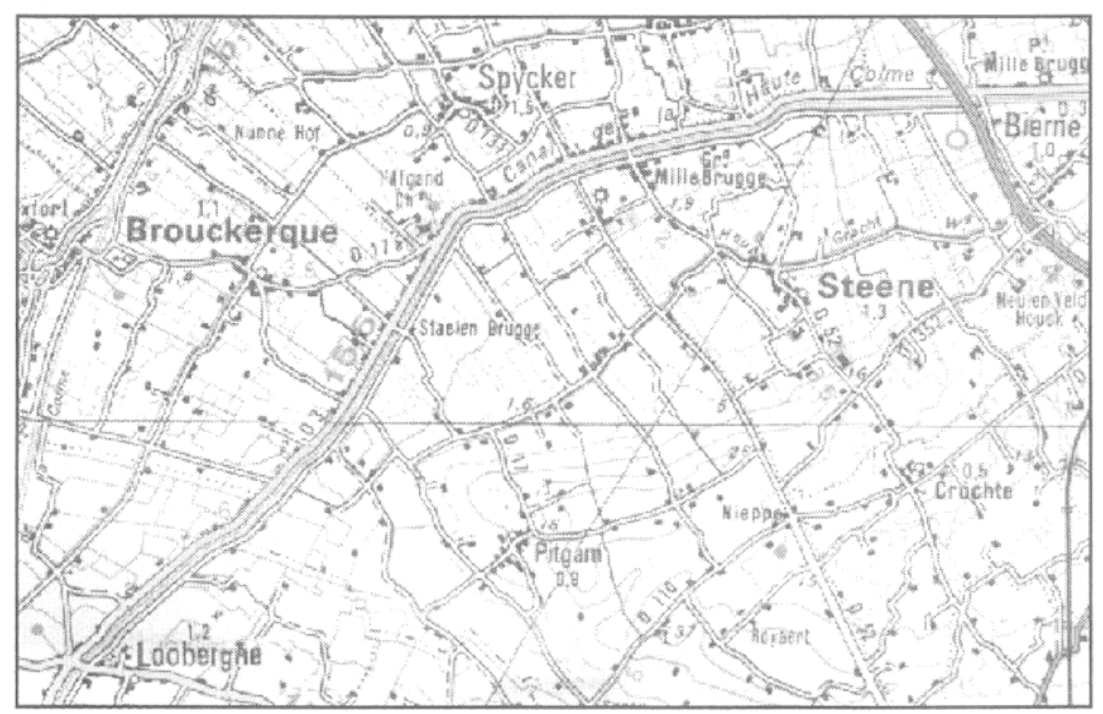

FIG. 1. - Localisation topographique des salines du littoral de la Flandre.

1. - Nous tenous à remercier chaleureusement notre ami Philippe Ducrocq de nous avoir donné l'occasion d'étudier la terre sigillée. Nous voulons aussi remercier les professeurs $H$. Thoen et J. Bourgeois
(Univ. Gand) pour leurs renseignements concernant le contenu et la forme du texte. 
tardives, n'est guère connu du point de vue structurel et socio-économique. A la recherche des publications de terre sigillée dans la région proche, nous n'avons trouvé que quelques études récentes (Ardres : CabalRetkowski, 1987; Cassel et Bailleul : De Clercq \& Deschieter, 1996 a \& b). Il en est de même pour les zones plus éloignées (Etaples, Arras, VendeuilCaply). Malheureusement très peu de ces publications ont traité le matériel de façon appropriée; à savoir une approche quantifiée, des dessins détaillés, des attributions les plus précises possibles. Ceci implique une analyse séparée de chaque motif et un maximum de critiques sur les données issues du matériel de surface ou de fouilles.

Sur une surface de quelques hectares, Ph. Ducrocq a localisé précisément différents " dépotoirs », dénommés de $\mathrm{S} 1$ à $\mathrm{S} 11$, en considération des concentrations de surface (fig. 1). Il a ramassé le matériel archéologique, en tenant compte de sa provenance par dépotoir. Cette approche détaillée de la prospection de surface n'a pas seulement donné lieu à la récolte d'une masse de poterie, mais a aussi abouti à une première idée concernant la localisation des différentes zones d'activités (zones artisanale ou d'habitat, zones de déchet). En ce qui concerne la chronologie du site de Steene-Pitgam, nous sommes pour le moment réduits à l'étude des trouvailles de surface. Hormis une quantité considérable de monnaies (détermination R. Delmaire), la plupart des trouvailles est composée de céramiques. Ces deux catégories, et dans une moindre mesure, le verre et les objets en métal (surtout des fibules) représentent les instruments nécessaires qui nous permettront de reconstruire le cadre chronologique du site de Steene-Pitgam. Les tessons recueillis embrassent tout le spectre céramique que l'on trouve généralement dans les sites gallo-romains du nord de la Gaule, notamment la céramique fine (terre sigillée, céramique gallo-belge, céramique engobée ou à enduit rouge-pompéien, poterie savonneuse...) et la céramique commune (gobelets, marmites, bols, assiettes, cruches, mortiers, amphores...). Chaque catégorie de matériel est en cours d'étude par différents spécialistes, cette contribution en étant le premier résultat.

\section{L'approche méthodologique}

L'étude d'un ensemble de céramique ne peut valoir que dans la comparaison avec d'autres trouvailles céramiques de la même région. A partir de cette étude comparative il devient possible de discerner certaines évolutions et tendances qui, à leur tour, reflètent le dévcloppcment chronologique et la hiérarchie socioéconomique des divers sites dans le même territoire. De plus, ces sites illustrent les mécanismes de com- merce et de distribution de la poterie gallo-romaine. Dans notre cas, le nord-ouest de la France comprend trois chefs-lieux de cités romaines : Cassel (cité des Ménapiens), Thérouanne (cité des Morins) et Arras (cité des Atrébates). Hormis ces agglomérations urbanisées on trouvait aussi des occupations rurales (Hornaing, Capelle-Fermont, etc.), artisanales (Labuissière, etc.), des villae (Bailleul, Noyelles Godault, etc.) et des sites aux affinités militaires (Boulogne).

Nous partons du principe que cette diversité d'occupation est déterminante pour la composition de l'ensemble de la céramique, et notamment de la terre sigillée. En ce qui concerne la quantité, la quantification, l'utilisation des attributions et des datations issues de nouvelles études et fouilles, on doit admettre que la recherche sur la terre sigillée dans la partie nord-ouest continentale de l'Empire romain, se trouve généralement fossilisée dans d'anciennes certitudes. En effet, la connaissance du matériel céramique est devenue, plus qu'auparavant encore, le privilège de quelques spécialistes, ce que nous regrettons. D'autre part, nous sommes conscients de la quantité de travail et de temps nécessaire pour traiter des ensembles de terre sigillée et de céramique commune, comprenant parfois des milliers de tessons. Aujourd'hui, vu la menace constante sur le patrimoine archéologique, la recherche archéologique se trouve dans la majorité des cas nécessairement limitée à l'archéologie de sauvetage. Cependant, les exemples sont légion, cette explosion du nombre et de l'étendue des fouilles, autant que la quantité de matériel mis au jour, aboutissent de moins en moins à des publications approfondies, faute d'argent et de temps. L'analyse de la céramique et des structures reste ainsi souvent limitée. En utilisant des "ouvrages de base », beaucoup d'archéologues affirment, sans le savoir et/ou par manque de renouveau des connaissances, des conceptions obsolètes, alors même que le site fouillé peut apporter des renseignements nouveaux. C'est dans ce cadre que nous voudrions plaider pour une revalorisation de l'étude de la céramique romaine, tant de la vaisselle de luxe que de la vaisselle commune. La fouille archéologique doit aboutir à des publications, se fondant sur des contextes bien clos, en évaluant et en considérant tous les éléments datables dans leur contexte (monnaies, amphores, sigillées, fibules...) et en utilisant les travaux de référence avec un maximum de critique. Bien que l'étude de la céramique commune gallo-romaine du nord de la France repose sur une longue tradition d'analyses systématiques (voir les nombreux articles et publications de M. TuffreauLibre et récemment Tuffreau-Libre \& Jacques, 1994), la terre sigillée n'a jamais bénéficié d'une attention 
comparable. Tout autant, comme dans le reste de la partie nord-ouest-continentale de l'Empire romain, la publication de contextes bien clos, statistiquement fiables et évalués de manière critique, n'est qu'un élément relativement récent et rare (pour le nord de la France : Thoen \& Seillier 1970; Bayard 1980; Hurtrelle \& Jacques 1983; Hosdez \& Jacques 1989; Loridant 1992; Bélot \& Canut 1995). Ce n'est donc pas la quantité du matériel qui importe, mais la qualité des contextes, voire l'association et la diversité du matériel trouvé qui peuvent apporter des renseignements. L'énorme quantité de données nouvelles issues des fouilles de la dernière décennie, structurelles autant que matérielles, a une valeur méconnue jusqu'à ce moment et nécessite donc une autre approche mćthodologiquc, voire critique.

Dans ce contexte, comment peut-on justifier une étude comme celle-ci, ne prenant en considération qu'une seule catégorie de poterie, provenant de prospections pédestres, par conséquent un matériel non stratifié et parfois très fragmenté ? Ce sont en premier lieu les fouilles qui doivent apporter des contextes bien datés qui, à leur tour, développent et esquissent le cadre de référence local, à presque tous les niveaux. Mais, devant une catégorie de matériel comme la terre sigillée, fossile directeur et témoin de l'unité culturelle et économique du monde gallo-romain par excellence, les comparaisons chronologiques et stylistiques du matériel même (datation, centre de production, potier) sont certainement valables sur un plan interrégional et international. Il n'en est pas de même pour la poterie locale et les différents modes de construction par exemple. Afin d'appliquer la terre sigillée comme indicateur chronologique ou économique sur le site de trouvaille, ou, autrement dit, la sigillée comme baromètre socio-économique et chronologique, un cadre de référence local, bien daté, statistiquement valable et surtout quantifié est préférable. Les régions n'ont pas toutes connu un développement économique ou social de la même intensité. De telles études sont rares pour la civitas Menapiorum et pour les parties frontalières des cités voisines (Nervii, Morini), mais nous voulons néanmoins prendre le risque de ne pas nous limiter à une description chronologique et stylistique du matériel (voir Catalogue) mais aussi, dans une certaine mesure, tenter d'utiliser la sigillée comme indicateur économique et chronologique pour le site. Comme nous nous fondons pour notre catalogue sur du matériel de référence souvent bien daté et quantifié, provenant de contextes militaires et civils en Allemagne, Angleterre, Belgique, France et aux Pays-Bas, il va de soi que nous sommes obligés de comparer notre essai d'analyse économique et chronologique avec ces sites lointains. Cette comparaison n'ira pas jusqu'à l'extrême : il nous suffit d'analyser les tendances générales. Finalement, tenant toujours compte qu'il s'agit du matériel de surface, nous voulons comparcr les données économiques avec l'étude de monnaies afin d'arranger ainsi une calibration méthodologique.

Précisons encore que la fiabilité statistique de la quantité du matériel en question ne posera pas de problèmes; il s'agit de 1333 tessons en sigillée, dont à peu près 130 pièces décorées et une trentaine de marques de potiers. Le nombre précis d'individus représentés est d'ailleurs difficile à vérifier : il y a d'abord l'aspect standardisé de la typologie qui empêche d'attribuer plusieurs tessons du même type à un individu, surtout quand il s'agit de trouvailles de surface. L'autre facteur concerne le caractère répétitif de la décoration : il est par exemple impossible de dire si les deux tessons 54 et 55 sont issus du même bol d'Advocisus. Beaucoup de tessons ont des cassures relativement fraîches et un grand nombre de pièces ont des dimensions assez grandes, ce qui nous entraîne à penser que l'état de conservation de certaines parties du site n'est plus tellement bon, peutêtre à cause du labour. Seules des fouilles peuvent apporter des renseignements plus solides à ce sujet et corroborer ou infirmer les hypothèses que nous allons proposer ci-dessous.

\section{LES AIRES DE PRODUCTION DES SIGILLÉES DE STEENE-PITGAM}

Il nous faut d'abord préciser que la détermination des pâtes, caractères si distinctifs pour les ateliers, a été réalisée de manière « macroscopique », c'est-àdire sans analyse chimique ou microscopique. En ce qui concerne les sigillées décorées, la forme, la décoration et l'attribution aux potiers sont déjà des éléments conclusifs afin d'obtenir une attribution fiable. Quant aux sigillées lisses, la situation est plus complexe. Les différents tessons ont été classés selon leur forme. Il va de soi que ces formes sont caractéristiques pour des périodes spécifiques : un Drag. 31 par exemple n'est pas produit dans le premier siècle. Mais, outre cette classification, nous avons comparé les tessons avec les pièces d'une collection de référence, comprenant des pièces estampillées et décorées, dont la provenance ne fait aucune doute. Finalement, les pièces douteuses ont été classées séparément. Il est également à noter qu'une certaine expérience dans la matière joue un rôle important à ce sujet.

Comme le montre le diagramme (fig. 2), les produits du sud de la Gaule ( ${ }^{\text {er }} \mathrm{s}$.) ne représentent que $8 \%$ du total. Les produits du centre (II $\left.{ }^{\mathrm{e}} \mathrm{s}.\right)$ 


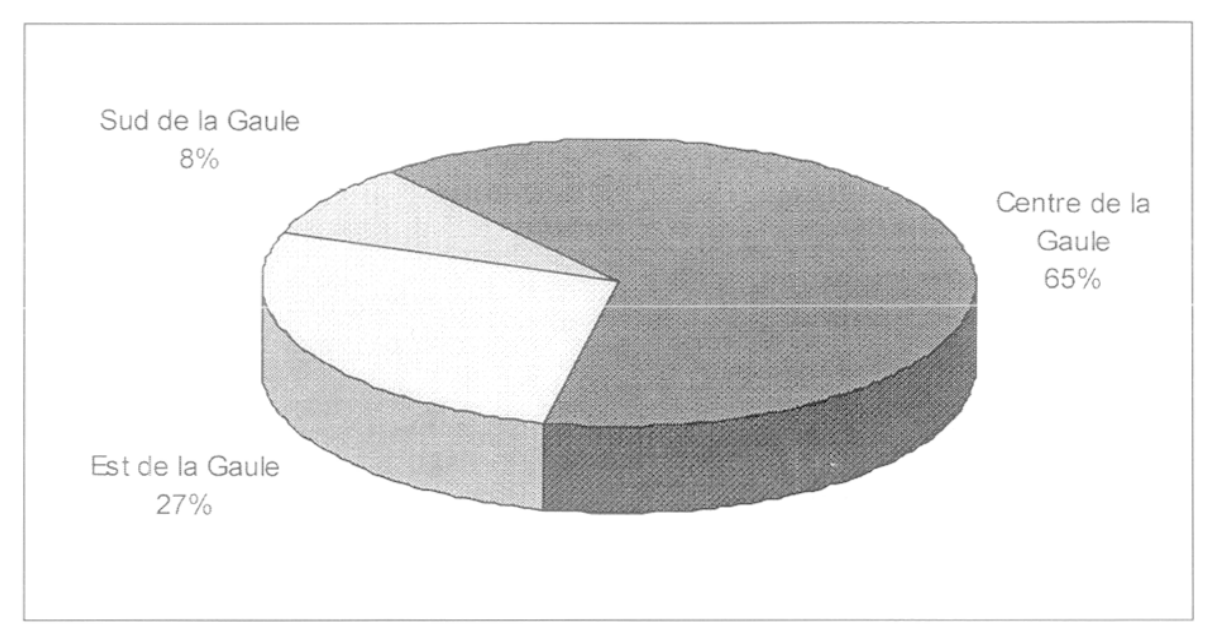

FIG. 2. - Distribution de l'ensemble des sigillées à base de pâtes.

représentent la majorité de l'ensemble, soit $63 \%$. Les tessons provenant de l'est de la Gaule (deuxième moitié II $^{\mathrm{c}}$ siècle et $\mathrm{III}^{\mathrm{e}} \mathrm{s}$.) représentent $28 \%$ du total. Les tessons de provenance incertaine (est ou centre de la Gaule) ne prennent que $1 \%$ du total. Les conséquences chronologiques seront spécifiées ci-dessous.

Les attributions détaillées (classification selon les officines respectives) n'ont été effectuées que pour le matériel estampillé et décoré. D'un autre point de vue, il est évident que dans les productions du sud de la Gaule, l'officine de La Graufesenque joue un rôle déterminant. Néanmoins, quelques pièces se rapprochent fort des productions de Banassac. Il s'agit vraisemblablement de pièces tardives (fin I $^{\text {er }}$ siècle et début $\left.\mathrm{II}^{\mathrm{C}}\right)$. Le matériel du centre de la Gaule est dominé par les produits de Lezoux, mais on ne peut pas exclure d'autres centres, comme par exemple Lubié et certainement Les Martres-de-Veyre (vers 100-120) minoritairement présents dans l'ensemble de Steene-Pitgam. La situation devient plus complexe pour les produits de l'est de la Gaule. Comme les différents petits centres de production se rapprochent fort par leur pâte des produits de Rheinzabern ou de l'Argonne, une identification sur base purement technique n'est donc guère permise. Il est également à noter que les productions trévires de la fin du $\mathrm{II}^{\mathrm{c}}$ et du début du $\mathrm{III}^{\mathrm{e}}$ siècle sont techniquement très proches de celles du centre de la Gaule. La situation à ce niveau est donc loin d'être simple. C'est pour cette raison que nous avons opté pour une division selon les différentes aires de production (est, centre et sud de la Gaule). Les attributions aux ateliers spécifiques n'ont donc été faites que pour les tessons décorés et estampillés, à l'exception de quelques pièces de technique indiscutable (comme par exemple les mortiers Dr. 45 de l'Argonne et de Trèves).

\section{LE RÉPERTOIRE FORMEL, LES MARQUES DE POTIERS ET LEURS APPORTS QUANTITATIFS ET CHRONOLOGIQUES.}

Dans le répertoire formel simplifié (fig. 3), un nombre d'à peu près 250 tessons (soit $20 \%$ du total) n'est pas attribuable, ce qui est évident, vu l'origine du matériel. Néanmoins, plus de 1000 tessons ont été classés selon leurs formes respectives. Les rapports entre les catégories typologiques sont relativement proches en ce qui concerne les tasses, les bols à collerette et les bols décorés. Seul le taux d'assiettes est écrasant. Mais, ici aussi, nous insistons sur la nature du matériel. Puisqu'il s'agit de matériel souvent très fragmenté, il est clair que les dimensions et l'épaisseur du vase jouent un rôle déterminant dans son degré de fragilité. A cet égard, il est évident que les assiettes (plus grandes que les tasses) se cassent plus rapidement. De plus, les pièces plus lourdes et plus épaisses, comme par exemple les mortiers Drag. 45 ou les bols décorés Drag. 37, se cassent fréquemment en des tessons plus grands. Cependant il est à noter que la catégorie des assiettes est constituée d'une multiplicité de formes, plus que pour les bols décorés par exemple. Ces remarques laissent supposer que les assiettes sont surreprésentées (fig. 3). Dès lors, nous voulons nous abstenir de conclusions trop poussées.

Quant à la typologie comme indicateur chronologique, les renseignements sont plus solides. La présence des formes Dr. 24/25 et Dr. 29 (forme décorée) et de certaines pièces des groupes Dr. 15/17, Dr. 27 et Dr. 18 indique une occupation pré-flavienne. Néanmoins nous devons admettre que cette présence n'est pas très importante; il s'agit de quelques pièces qui datent de l'époque Claude-Néron. Les conséquences socio-économiques seront traitées plus loin. 


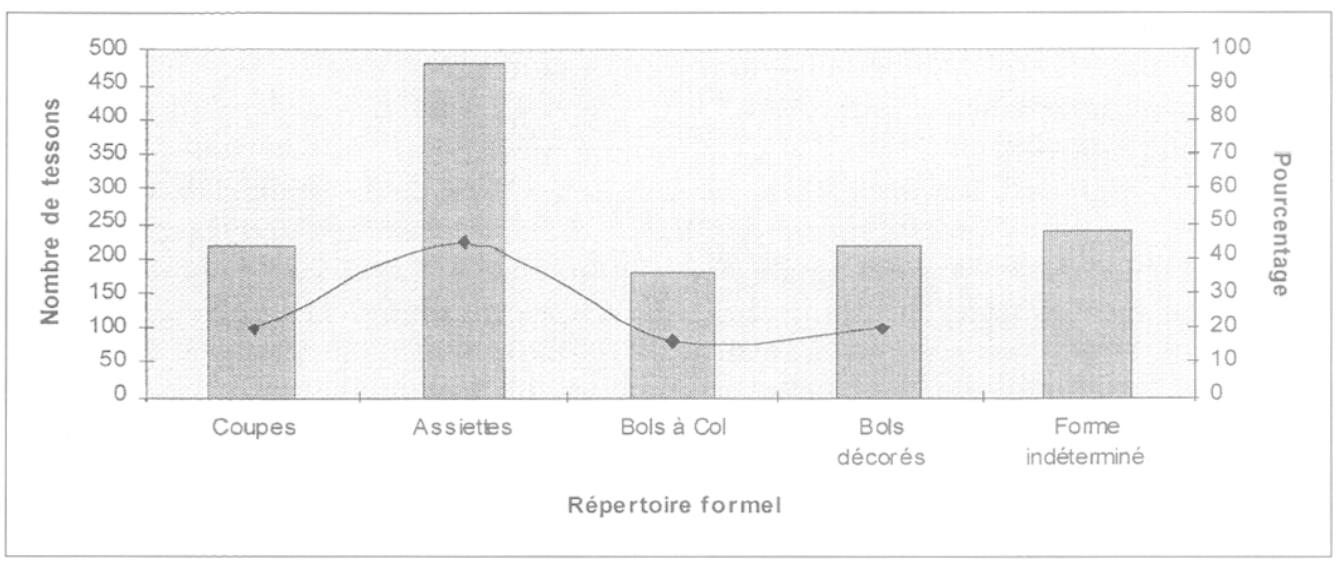

FIG. 3. - Division typologique sur base des senices.

La majorité de la sigillée unie du sud de la Gaule date sans doute de l'époque flavienne : ce sont surtout les formes Dr. 18 et Dr. 27 qui sont bien représentées. Cette constatation est de plus confirmée par les proportions relatives entre les types Dr. 18 et 27 et les types Dr. $15 / 17$ et $24 / 25$. Les premiers constituent en effet des remplacements simplifiés et tardifs des autres.

Parmi les marques de potiers, seules deux estampilles d'ateliers méridionaux ont été trouvées. Il s'agit d'une marque de Secundus de La Graufesenque (date globale : Néron-Flaviens) et d'un début d'estampille avec un « $O$ » sur Dr. 15/17, datant de l'époque Claude-Néron. Signalons encore un exemplaire de Ritterling 9 du sud de la Gaule. Ce type relativement rare a un bord profilé et arrondi. La forme date généralement de l'époque pré-flavienne (Tibère-Claude).

La majorité des sigillées unies provient des officines du centre de la Gaule. Dans la première moitié du II $^{\mathrm{e}}$ siècle, cette prédominance est absolue, hormis peut-être quelques pièces tardives du sud de la Gaule (provenant par exemple de Banassac), datant du début du siècle. L'uniformité typologique et la production de masse de quelques formes, avec une prédilection pour les formes plus simples aux parois plus épaisses, la diminution du nombre de cannelures, etc. sont des éléments caractéristiques pour les productions de cette époque.

Néanmoins on peut dégager quelques constatations. Dans le groupe des tasses et coupes, le Dr. 33 domine largement le Dr. 27. Plus particulièrement, le Dr. 33 à profil concave est bien représenté. Ces formes datent surtout de l'époque antonine (milieu et deuxième moitié $\mathrm{II}^{\mathrm{C}}$ siècle). Parmi les formes les plus rares, il faut mentionner la forme Oswald \& Pryce LV, 13 : une tasse à lèvre pendante, à parois légèrement concave et fond - presque exclusivement - estam- pillé en forme de rosette. Cette forme date du milieu et du troisième quart du $\mathrm{II}^{\mathrm{e}}$ siècle. Parmi les assiettes, ce sont surtout les formes Dr. $18 / 31$ et 31 qui dominent. Vu l'état du matériel, nous avons opté pour la distinction entre deux catégories principales : un groupe avec les formes Dr. 18/31 ou 31 (la différence est souvent difficile à faire sur base du matériel fragmentaire) et une catégorie comprenant les formes Dr. 31. Les Dr. 31 du centre de la Gaule sont caractéristiques pour la deuxième moitié du $\mathrm{II}^{\mathrm{e}}$ siècle. De plus, nous sommes convaincus qu'il y a dans le groupe 18/31 ou 31 un certain nombre de Dr. 31. Finalement, les formes moins courantes comportent des types Walters 79 ou 80 (dernier tiers $\mathrm{II}^{\mathrm{e}}$ siècle) et Curle 15 (deuxième moitié $\mathrm{II}^{\mathrm{e}}$ siècle). Une autre forme plus rare est l'assiette type Bet 47 . Ce récipient caréné appartient à la période flavienne et au début du $\mathrm{II}^{\mathrm{e}}$ siècle (Bet \& Montineri, 1990).

La plupart des estampilles de potiers (25 sur 27) provient des ateliers du centre. Douze marques sont lisibles, cinq restent illisibles. Les potiers identifiés ont travaillé à Lezoux. Il s'agit d'Albucius, Certus, Genialis, Ianuarius, Macro, Mainacnus, Malledo, Masvetus, Nicephor, Reogenus, Santianus, Saturninus et peut-être Doeccus. Tous ces potiers peuvent être datés de la deuxième moitié du II $^{\mathfrak{e}}$ siècle.

Pour le matériel de l'est de la Gaule, la présence des assiettes Dr. 31, Wa 79/80 et des tasses Dr. 33 est indiscutable. La datation se situe dans la deuxième moitié du $\mathrm{II}^{\mathrm{e}}$ siècle et la première moitié du $\mathrm{III}^{\mathrm{e}}$ siècle. Les types datant exclusivement du III $^{\mathrm{E}}$ siècle sont absents. Néanmoins, les bols à collerette sont bien présents. Il s'agit des Dr. 38 (tous du centre de la Gaule à Steene-Pitgam) et surtout une grande quantité de mortiers Dr. 45 (ou 43), presque exclusivement en technique de l'est de la Gaule, voire de l'Argonne. Pour des raisons purement typologiques, nous avons 
fait la distinction entre les Dr. 45 ou 43 d'une part et les Dr. 45 certains de l'autre. En réalité il est clair que la majorité de ces tessons appartient à la forme Dr. 45, ce type étant largement plus fréquent que le Dr. 43. Le mortier Dr. 45 est surtout produit dans les ateliers de l'est (Rheinzabern, Argonne et Trèves), encore que nous possédions quelques pièces du centre de la Gaule. La datation du type se situe dans le dernier quart du $\mathrm{II}^{\mathrm{e}}$ et tout le $\mathrm{III}^{\mathrm{e}}$ siècle. D'un point de vue technique, la majorité des Dr. 45 provient des ateliers de l'Argonne. Cette forte proportion des produits argonnais dans la sigillée de l'est - Trèves et surtout Rheinzabern sont moins représentés, même dans le matériel décoré - est tout à fait remarquable et peut s'expliquer de différentes raisons (voir plus bas). Quant à la dominance du type Dr. 45, nous sommes d'avis de considérer l'aspect formel. Il est frappant de trouver dans les sites du nord de la France et dans les régions voisines une quantité impressionnante de Dr. 45; nous nous référons par exemple aux sites de Harnes (Notte, 1990), Ardres (Cabal, 1962-1967), Watten (Voet, 1983) et Braives (Bet \& Brulet, 1994). Cette prédominance peut s'expliquer de plusieurs façons. Peut-être existait-il une sorte de préférence régionale, tant pour la forme que pour le type de repas que l'on préparait ou mangeait. Une autre explication - plus plausible, nous devons l'admettre - est d'ordre chronologique. Le III $^{\mathrm{c}}$ siècle se caractérise par une simplification du répertoire formel, aboutissant à quelques types (Dr. 31, 32, 40 et 45) dominant le marché. Dans ce cadre, il serait possible de voir la présence dominante des Dr. 45 comme un simple témoin de la typologie de la sigillée du $\mathrm{III}^{\mathrm{c}}$ siècle. Dans le camp de la Classis Britannica à Douvres, il semble que les Dr. 45 soient importés plus longtemps que les autres formes (Bird \& Marsh, 1981, p. 178-179). D'autre part, il est vraiment frappant que les formes typiques du III ${ }^{\mathrm{e}}$ siècle comme les Dr. 32 et 40 soient tout à fait absentes à Steene-Pitgam. Il en est de même pour les Dr. 37 de la même époque (infra). Une dernière cxplication se trouve peut-être dans la nature de l'approvisionnement même. Comme nous le démontrerons plus loin, il semble que les sites du nord de la France aient connu des liens commerciaux très étroits avec les ateliers de l'Argonne, et que ceux-ci se soient emparés d'une bonne partie du marché régional à partir de la deuxième moitié du II $^{\mathrm{c}}$ siècle. Comme le Dr. 45 était particulièrement abondant dans les productions de l'Argonne à la fin du $\mathrm{II}^{\mathrm{c}}$ et au $\mathrm{III}^{\mathrm{c}}$ siècle, il est donc facile d'expliquer pourquoi ils dominent le spectre. Nous attendons d'autres analyses et surtout des contextes régionaux bien datés pour examiner ce phénomène plus en profondeur.

Quant aux formes rares, la présence d'un tesson de paroi peut-être d'un canthare Ludowici VMd ou VMe, décoré d'une feuille appliquée à la barbotine, est remarquable. Cette pièce peut être datée de la deuxième moitié du II $^{\mathrm{e}}$ siècle ou première moitié du $\mathrm{III}^{\mathrm{e}}$ siècle. Les canthares ont toujours étés considérés comme des outils rituels. Dans la majorité des cas, ils ont été trouvés sur des sites militaires. Les militaires ont joué un rôle important dans la dispersion des cultes mystiques orientaux comme par exemple le culte de Mithra au III $^{\mathrm{c}}$ siècle. Cependant cette dispersion n'est pas uniquement axée sur des sites militaires comme le montrent quelques exemples en Belgique (Thoen \& De Swaef, 1995).

Finalement, aucune marque de potier ne date $\mathrm{du}_{\mathrm{III}}{ }^{\mathrm{s}}$ siècle, ce qui s'explique non seulement par la prédominance des pièces du centre de la Gaule mais aussi par le fait que l'habitude d'estampiller les céramiques devient de moins en moins courante à cette époque.

\section{LA SIGILLÉE DÉCORÉE ET SON APPORT À LA CHRONOLOGIE ET À L'ÉCONOMIE DU SITE (FIG. 4-7)}

Au total, 98 pièces ont été attribuées à un potier ou à un groupe de potiers. Cette attribution concerne toutes les pièces lisses estampillées et les vases décorés. Puisqu'il s'agit de matériel parfois très fragmenté, 29 tessons n'ont pu être attribués que de façon générale à une période de manufacture (par exemple : flavien, antonin tardif, etc.). Le tableau (fig. 4) résumant toutes ces données a été conçu suivant la méthode décrite par Greene (1975, p. 66-67). Cette méthode de quantification chronologique est basée sur l'hypothèse qu'un tesson, à titre d'exemple daté entre 150 et 180 , est présent dans les catégories des années 150$160,160-170$ et 170-180. L'application de cette méthode nous permettra de faire des comparaisons avec les données issues des sigillées des sites traités de la même façon (Marsh 1981, Bird 1986, Piton 1990).

Comme déjà indiqué lors de l'analyse du répertoire formel, la période pré-flavienne n'est guère représentée dans l'ensemble des pièces décorées. On a toutefois reconnu un bord de Drag. 29 et un tesson de paroi de la même forme (potier inconnu). Ces deux pièces datent de l'époque Néron-Vespasien. Un fragment de Dr. 30 est attribué au style de Masclus ou un de ses contemporains, et date de l'époque Claude-Néron. Ce n'est qu'à l'époque flavienne que commence l'importation plus au moins massive de la sigillée décorée sur le site. La forme la plus courante est le bol hémisphérique Dr. 37 dont quatre ont été exécutés dans les styles de Severus, Mercato et Germanus III. L'organisation de leurs décors suit les schémas en usage à La Graufesenque à l'époque flavienne. Quelques bols datent plus spécifiquement du règne de Domitien, donc des deux dernières décennies du 


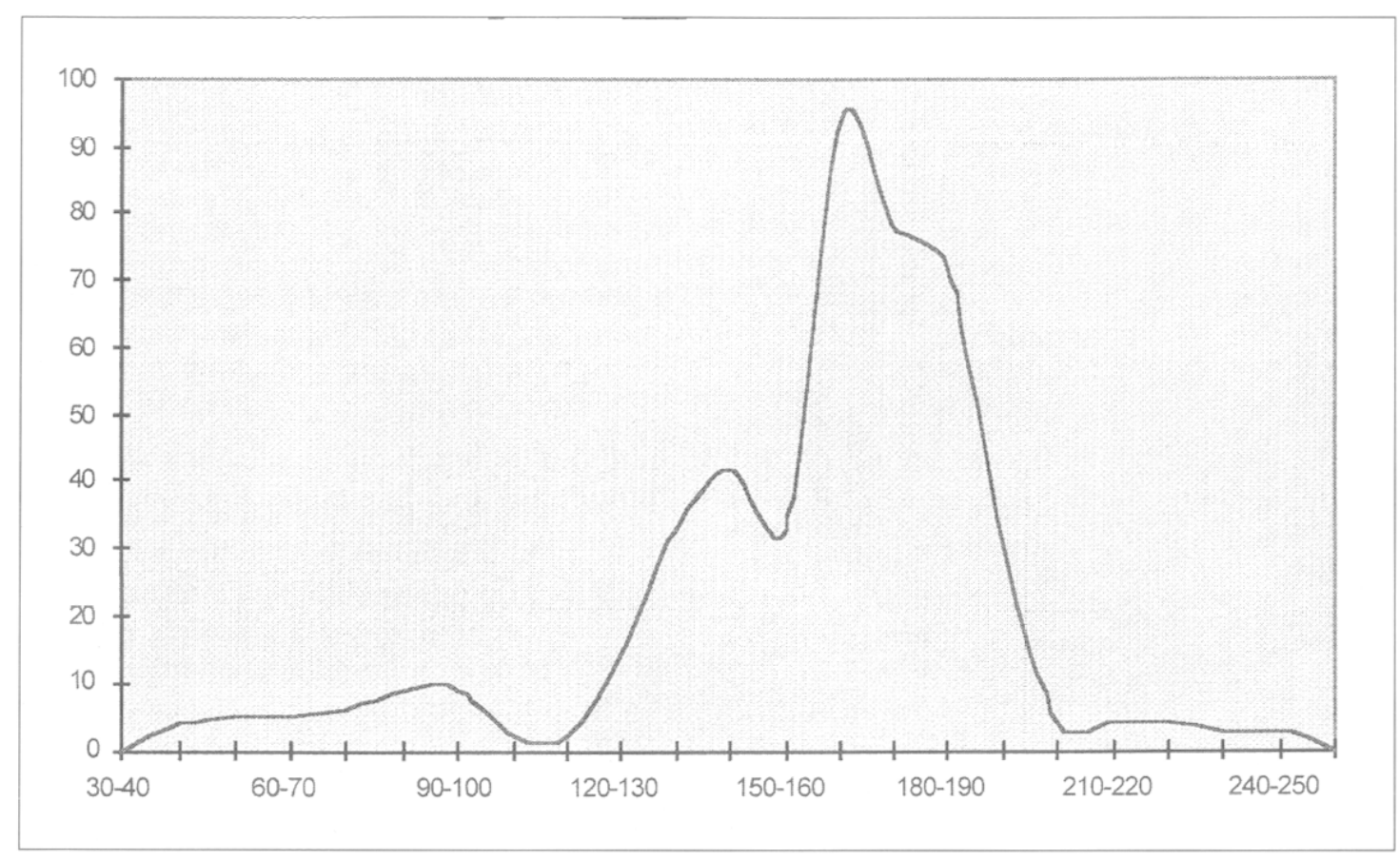

FIG. 4. - Approvisionnement en sigillée : distribution chronologique des pièces attribuées (estampilles et vases décorés).

$\mathrm{I}^{\mathrm{er}}$ siècle.

Sous le règne de Trajan, les ateliers du centre de la Gaule prennent le relais. Peu de tessons de SteenePitgam appartiennent à cette période. L'absence des produits décorés des potiers des Martres-de-Veyre, comme X-1, X-2, Drusus I et Igocatus, nous semble d'ailleurs significative. Ce fait n'a vraisemblablement rien à voir avec des événements sur le site même. Le changement d'aire de production et l'entrée sur le marché inter-régional des nouvelles officines ne s'est apparemment pas produit sans problèmes de distribution : la courbe montre ainsi une baisse (infra: conclusion).

Dès l'époque d'Hadrien, le nombre de vases moulés ornés croît sans cesse et cela jusqu'au début du dernier quart du siècle. A cette époque (vers 117-145) les potiers distincts comme le groupe autour AvitusVegetus et Quintilianus d'une part et les potiers X-6, Attianus, Secundinus I et Birrantus de l'autre, sont bien présents à Steene-Pitgam. Tous ces bols se caractérisent par un finissage très soigné à surface brillante, une pâte assez fine, une cuisson dure et un arrangement de décor bien élaboré et souvent de style baroque, rappelant les éléments du répertoire stylistique du sud de la Gaule comme par exemple les croix de Saint-André, les bandes de bi- et trifols et les rinceaux de feuilles élaborées. A partir du règne d'Antonin le Pieux, les produits de masse des ateliers de Lezoux et de ses environs atteignent le site sous la forme de Dr. 37 et 30 . Il s'agit de bols aux métopes et en style libre (surtout des scènes de chasse) de Criciro et du groupe Criciro-Aventinus, de Satus-Cettus (Les Martres-de-Veyre), Pugnus et de Divixtus. Ce dernier se trouve représenté par trois bols différents, entre autres un Dr. 37 exécuté dans son style distinctif aux caryatides. Cette période se caractérise aussi par l'apparition massive sur le marché des bols de l'atelier de Cinnamus. Ses produits précoces (ove 3 ; trois pièces au minimum) sont souvent réalisés en association avec le décorateur Cerialis. La datation de la phase de production de cette fameuse officine, industrielle avant la lettre, a été précisée vers 135-145 (Simpson et Rogers, 1969 , p. 3-14 : vers 150-165 ; Rogers, 1969 1970 , p. $98-106$ : vers 145 et finalement Rogers 1972 , p. 322-326 : vers 135-145). Les autres pièces de Cinnamus se situent vers 135-170 et sont attestées sur 11 pièces. Il s'agit de Dr. 37 et 30 au style distinct aux métopes et aux festons, à estampillage prononcé, une disposition de l'espace bien arrangée et équilibrée, voire linéaire. Quelques fragments sont attribués sous bénéfice d'inventaire à Tetturo, Fgientinus et Paullus. Dans le groupe antonin moyen nous insistons encore sur la présence des quatre bols d'Advocisus.

La majorité des bols décorés de l'ensemble est composée de pièces de la période antonine tardive (vers 170-200). Il s'agit de produits du groupe de Censorinus-Paternus II-Laxtucissa (14 pièces), de 


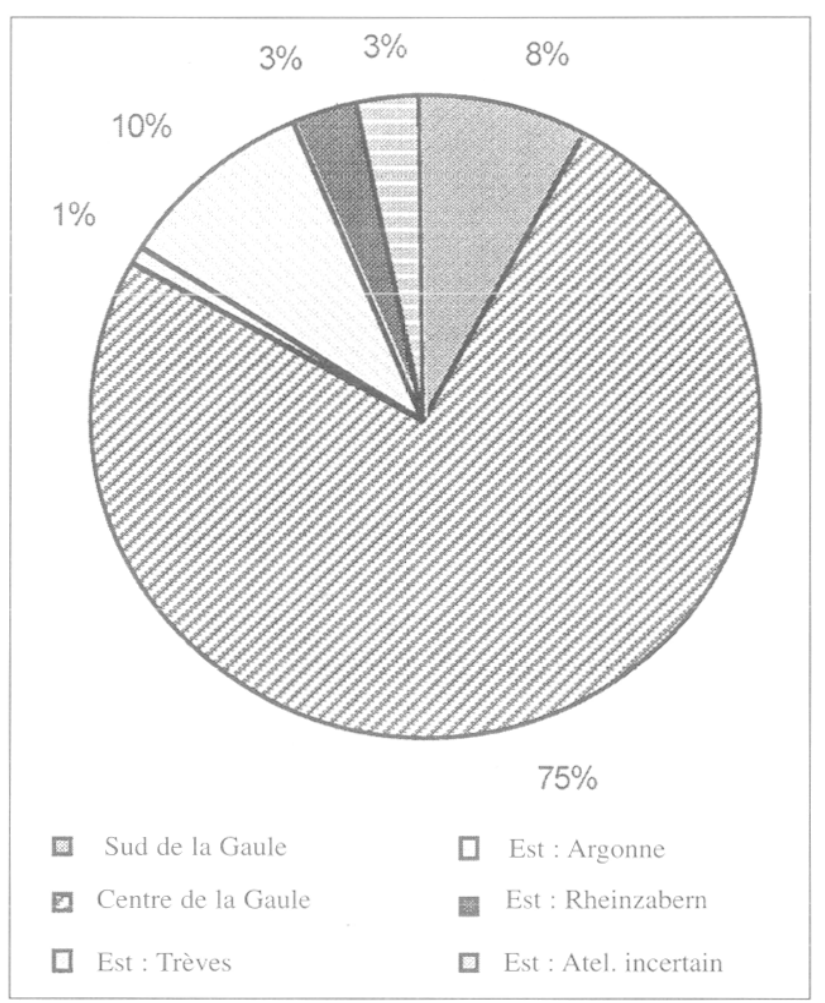

FlG. 5. - Distribution de l'ensemble des sigillées attribuées (estampilles et décorés) selon les aires de production.

Iustus, Iullinus, Severus-Caletus, Servus II et des potiers souvent associés Casurius (9) et Doeccus (7). Les descriptions détaillées sont données dans le catalogue et nous nous contentons de mentionner le style spécifique de Casurius et Doeccus. L'oeuvre du potier Casurius se caractérise par un estampillage vraiment très prononcé, avec un arrangement du décor en métopes aux larges perles arrondies et aux motifs bien élaborés, souvent répétitifs. Cela vaut également pour le potier Doeccus à la différence que, chez lui, les perles sont plus souvent carénées et que son style, entre autres avec festons et médaillons, se rapproche de la décoration employée par Cinnamus. Précisons encore, que la majorité des bols décorés dans la période 150-200 ne sont produits que dans les officines de quelques potiers comme Cinnamus, Casurius/Doeccus et le groupe de CensorinusPaternus II-Laxtucissa.

C'est également à cette époque que les produits de l'est de la Gaule font leur apparition. En ce qui concerne le répertoire uni. la présence d'un nombre assez important de pièces de l'Argonne est remarquable (fig. 5 et 6). Faute d'une analyse approfondie du matériel issu de fouilles anciennes, la connaissance de la sigillée mais aussi des potiers de l'Argonne est

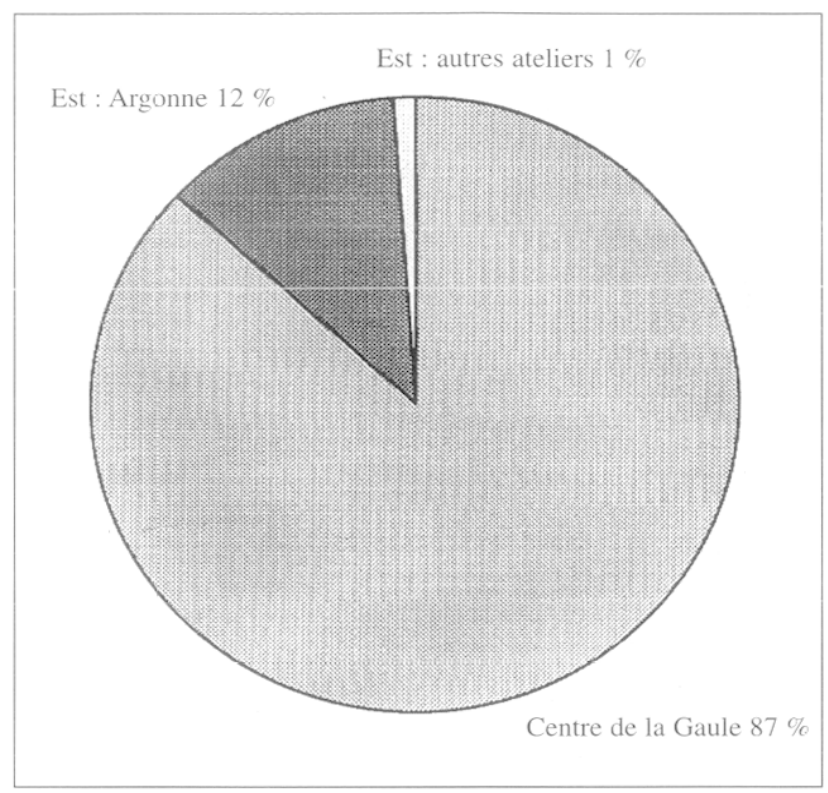

FIG. 6. - Approvisionnement en sigillée dans le deuxième siècle (estampilles et sigillée décorée).

encore insuffisante. Nous ne possédons que quelques catalogues et ensembles bien étudiés (Hofmann, 1968; Chenet \& Gaudron, 1955, Raepsaet-Charlier \& Clausse, 1977-1978). La majorité des pièces trouvées à Steene-Pitgam date de la deuxième moitié du II $^{\mathrm{e}}$ siècle. Parmi les potiers reconnus, on peut citer Eburus et le groupe des potiers Tribunus, Germanus et $T$ occa, ainsi que le potier à l' " ove $\mathrm{G}$ ». Après la fin du II' siècle, l'approvisionnement en sigillée décorée et estampillée retombe à un niveau comparable à celui de l'époque flavienne précoce. Les raisons seront traitées dans la conclusion.

Pour le III $^{\mathrm{e}}$ siècle, nous trouvons quelques pièces des officines trévires: à savoir le groupe AferMarinus (vers 200-225) et Dubitatus (225-250). Les bols de Rheinzabern sont représentés à Steene-Pitgam par les produits des potiers Comitialis V, Victor I et Julius II-Julianus. Comitialis est datable de la fin du II $^{\mathbb{E}}$ et du début du III $^{\mathbb{C}}$ siècle, Julius II-Julianus I travaillaient après 225 et Victor I était actif dans la première moitié du III $^{\circ}$ siècle (Simon, 1968, 22 et Bernhard, 1981 Abb.3). L'ensemble de la terre sigillée décorée ne contient aucune pièce que l'on puisse assigner au Bas-Empire. 


\begin{tabular}{|c|c|c|c|}
\hline$\frac{\text { Potiers attribués (sigillée }}{\text { décorée et estampilles). }}$ & Atelier & Nombre & Datation approximative \\
\hline \multicolumn{4}{|l|}{ Epoque pre-Agvienne } \\
\hline $\begin{array}{l}\text { Masclus } \\
\text { potiers incertains }\end{array}$ & LG & 1 & ca $40-70$ \\
\hline & & $\ldots$ & ca $40-1$ \\
\hline \multicolumn{4}{|l|}{ Elavien } \\
\hline Secundus $11^{*}$ & LG & 1 & ca $55-100$ \\
\hline Severus & $\mathrm{LG} / \mathrm{BA}$ & i & ca $70-100$ \\
\hline Germanus III & LG & 1 & ca $70-100$ \\
\hline Mercato** & LG & 1 & ca $80-100$ \\
\hline Mercato ou Calus & LG & 1 & ca $80-100$ \\
\hline potiers incertains: flavien & & 3 & ca $70-100$ \\
\hline $\begin{array}{l}\text { potiers incertains: flavien } \\
\text { tardif }\end{array}$ & - & 1 & ca $80-110$ \\
\hline \multicolumn{4}{|l|}{ Hadrien-Antonin précoce } \\
\hline Secundinus $\mathrm{I}$ & LZ & 1 & ca $110-140$ \\
\hline Avitus-Vegetus & LZ & 2 & ca $120-150$ \\
\hline Sacer & $\mathrm{LZ}$ & $i$ & ca $120-140 / 5$ \\
\hline Attianus & $\mathrm{LZ}$ & 2 & ca $120-140 / 5$ \\
\hline Attianus ou Acaunissa & $\mathrm{LZ}$ & 1 & ca $120-140 / 5$ \\
\hline $\begin{array}{l}\text { X-6 } \\
\text { Macro* }\end{array}$ & $\begin{array}{l}\mathrm{L} Z \\
\mathrm{LZ}\end{array}$ & $\begin{array}{l}4 \\
1\end{array}$ & $\begin{array}{l}\text { ca } 125-145 / 50 \\
\text { ca } 125-150\end{array}$ \\
\hline Birrantus & LZ & 1 & ca $125-150$ \\
\hline lanuarius II * & LZ & 1 & ca $125-155$ \\
\hline $\begin{array}{l}\text { potiers incertains: Antonin } \\
\text { précoce }\end{array}$ & - & 4 & ca $120-140 / 5$ \\
\hline \multicolumn{4}{|l|}{$\frac{\text { Antonin précoce à Antonin }}{\text { moyen }}$} \\
\hline Criciro-Aventinus & $\mathrm{LZ}$ & 1 & ca $130-150$ \\
\hline Criciro & $\mathrm{LZ}$ & 1 & ca $130-150$ \\
\hline Satus-Cettus & MV & $i$ & ca $135-160$ \\
\hline Cinnamus-Cerialis / ove 3 & LZ & 3 & ca $135-145$ \\
\hline Cinnamus: autres oves & LZ & 12 & ca $135-170$ \\
\hline Tetturo & $\mathrm{LZ}$ & 1 & ca $135-165$ \\
\hline Nicephor II * & $\mathrm{LZ}$ & 1 & ca $140-155$ \\
\hline Divixtus & $\mathrm{LZ}$ & 3 & ca $140-160$ \\
\hline Certus II * & $\mathrm{LZ}$ & 1 & ca $140-165$ \\
\hline Pugnus & LZ & 2 & ca $140-170$ \\
\hline Servus III & LZ & 3 & ca $140-160$ \\
\hline Sissus II & 1.7 & 1. & ca $145-170$ \\
\hline \multicolumn{4}{|c|}{ Antonin tardif et fin deusième siècle (centre el est) } \\
\hline Albucius il * & $\mathrm{LZ}$ & 1 & ca $150-160$ \\
\hline Potier & Atelier & Nombre & Datation \\
\hline Malledo. & $\mathrm{LZ}$ & 1 & ca $150-180$ \\
\hline Masvetus II * & LZ & i & ca $150-180$ \\
\hline Santianus* & LZ & i & ca $150-180$ \\
\hline Iustus & LZ & $i$ & ca $150-180$ \\
\hline Reogenus * & LZ & $i$ & ca $150-180$ \\
\hline Saturninus II * & LZ & $i$ & ca $160-180$ \\
\hline Mainacnus** & $\mathrm{LZ}$ & 1 & ca $160-200$ \\
\hline Cintinus & LZ & 1 & ca $160-200$ \\
\hline Genialis IV * & LZ & $i$ & ca $160-195$ \\
\hline Advocisus & LZ & 4 & ca $160-190$ \\
\hline Groupe Censorinus-Paternus & LZ & 13 & ca $160-190$ \\
\hline $\begin{array}{l}\text { II-Laxtucissa } \\
\text { Paternus II ** }\end{array}$ & $\mathrm{LZ}$ & 1 & ca $160-190$ \\
\hline Iullinus & LZ & 2 & ca $160-190$ \\
\hline Severus-Caletus & $\mathrm{LZ}$ & 1 & ca $160 \cdot 190$ \\
\hline Servus II & LZ & i & ca $160-190$ \\
\hline Casurius & $\mathrm{LB}+\mathrm{LZ}$ & 9 & ca $160-195$ \\
\hline Doeccus & $\mathrm{LZ}$ & 7 & ca $160-195$ \\
\hline Eburus (?) & $\mathrm{AR}$ & 1 & ca $150-200(?)$ \\
\hline ove $\mathrm{G}$ & $\mathrm{AR}$ & 4 & ca $175-200$ \\
\hline $\begin{array}{l}\text { Groupe Tribunus-Germanus- } \\
\text { Tocca }\end{array}$ & $\mathrm{AR}$ & 4 & ca $160-190(?)$ \\
\hline $\begin{array}{l}\text { Tocca } \\
\text { potiers incertains: antonin } \\
\text { tardif (ccntrc et cst) }\end{array}$ & & 23 & ca $160-190$ \\
\hline \multicolumn{4}{|l|}{$\frac{\text { Fin deuxième-début troisième }}{\text { siècle }}$} \\
\hline $\begin{array}{l}\text { Comitialis V } \\
\text { potier incertain }\end{array}$ & $\begin{array}{l}\mathrm{RZ} \\
\mathrm{RZ}\end{array}$ & 1 & $\begin{array}{l}\text { Fin II - Déb. IIII } \\
\text { Fin II - Déb. III }\end{array}$ \\
\hline \multicolumn{4}{|l|}{$\frac{\text { Premier quart troisième }}{\text { siècle }}$} \\
\hline Afer-Marinus & TR & 1 & ca $200-225$ \\
\hline \multicolumn{4}{|l|}{$\frac{\text { Deuxième quart troisième }}{\text { siècle }}$} \\
\hline $\begin{array}{l}\text { Julius II-Julianus I } \\
\text { Dubitatus }\end{array}$ & $\begin{array}{l}\mathrm{RZ} \\
\mathrm{TR}\end{array}$ & 1 & $\begin{array}{c}\text { ca } 225-245 / 250 \\
\text { ca } 225-250\end{array}$ \\
\hline \multicolumn{4}{|l|}{$\frac{\text { Premier moitié troisième }}{\text { siècle }}$} \\
\hline Victor I & $\mathrm{RZ}$ & 1 & ca $200-250$ \\
\hline
\end{tabular}

FIG. 7. - Liste des potiers attribués (sigillées décorées; estampilles* sur sigillée unie; estampilles** sur sigillée décorée).
Liste des marques sur sigillée unie ${ }^{3}$ : estampilles identifiables (en ordre alphabétique)

1. (S2). Dr. 18/31 : [AL]BVCøI : Albucius II de Lezoux, vers 150160 après J.-C.

2. (S7). Dr. 33 : CI[IR]TIML : Certus II de Lezoux, vers 140-165. 3. (S7). Dr. 33 : GENIALI [ : Genialis IV de Lezoux, vers 160190.

4. (S7). Dr. 33 : [I]ANUAR[I] : Ianuarius II de Lezoux, vers 125 155.

5. (S2). Dr. 18/31 : [M]ACRO phallus : Macro de Lezoux, vers 125-150.

6. (S1). Dr. 31 : MAINACN : Mainacnus de Lubié et de Lezoux, vers $160-200$.

7. (S1). Dr. 33 : MALLEDVF : Malledo de Lezoux, vers 150-180.

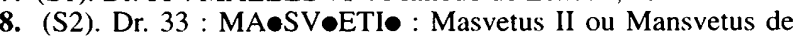
Lezoux, vers $150-180$.

9. (S2). Dr. 18/31 : [NICE]PHOR॰F : Nicephor II de Lezoux, vers 140-155.

10. (S1). Dr. 31 : RI IOGEN[IM] : Reogenus de Lezoux, vers 150180 .

11. (S2). Walt.79 (brûlé) : SANTIANIM : Santianus de Lezoux, vers $150-180$.

12. (S1). Dr. 33 : SATVRNINI : Saturninus II de Lezoux, vers 160 180.

13. (S3). Dr. 27 : SEC $\bullet V N D I$ : Secundus II de La Graufesenque (Néron-Domitien).

\section{Marques non-identifiables :}

14. (S2). Dr. $15 / 17$ ou 18 (sud de la Gaule) : O[ : Claude-Néron. 15. (S5). Dr. 27 : (sud de la Gaule) : TVM[ Flavien.

16. (S1). Dr. 27 : (centre de la Gaule) : M[ : Hadrien-Antonin précoce.

17. (S1). Dr. ? : (centre de la Gaule) : DOI : il s'agit peut-être de Doeccus de Lezoux, 165-200.

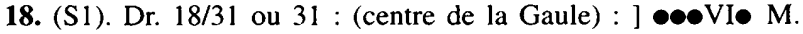
Deuxième moitié II $^{\mathrm{e}} \mathrm{s}$.

19. (S9). Dr. $31 \mathrm{R}$ (centre de la Gaule) : ] MVS : Deuxième moitié II $^{\mathrm{e}} \mathrm{s}$.

20. (S1). Dr. 33 (centre de la Gaule) : marque anépigraphique : Deuxième moitié II $^{\mathrm{e}} \mathrm{S}$.

21-27 : Restent encore 7 marques de potiers, complètement illisibles.

\section{Graffiti.}

1. (S2). Dr. 33 (Trèves) : « B »

2. (S1). Dr. $31 \mathrm{R}$ (Trèves) : «V »

3. (S7). Dr. 33 estampillé GENIALI (supra) : « $X$ »

4. (S1). Dr. 18/31R ou 31R (centre de la Gaule) estampillé

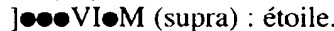

2. — LG : La Graufesenque : BA : Banassac ; LB : Lubié : LZ : Lezoux : TR : Trèves : RZ : Rheinzabern : AR : Argonne : MV : Martres-de-Veyre.

3. - L'attribution, la classification et la datation des estampilles sur sigillée unie sont basées sur le nouvel Index of Potters' Stamps on Samian Ware (Terra Sigillata) (voir : Hartley, 1993). Les estampilles de Certus, Ianuarius et Reogenus ne sont pas illustrées. Il s'agit de pièces récemment trouvées et pour lesquelles le temps de les dessiner nous a manqué. 


\section{v. CONCLUSION}

Lors des prospections de surface à Steene-Pitgam, 1333 tessons en sigillée ont été ramassés à onze endroits différents sur un site saunier en bordure de la plaine maritime. Les dépotoirs S1 et S2 ont livré la majorité de la sigillée : 1040 tessons, soit $78 \%$ du total. La présence de différentes aires de production dans les différents dépotoirs est similaire, sauf peutêtre pour le dépotoir $\mathrm{S} 9$ où on a constaté que 10 tessons sur 27 sont du sud de la Gaule (37\%), $14 \mathrm{du}$ centre et 3 de l'est, et sur le dépotoir $S 8,4$ tessons sur 11 sont originaires du Sud de la Gaule. Ce nombre important (en pourcentage) des produits méridionaux est en contradiction avec les données des autres dépotoirs.

L'ensemble comprend une multiplicité de formcs ct de techniques. Dans cette contribution, nous avons essayé, non seulement d'analyser les tessons sur base de leur technique, forme et décoration, mais aussi de les utiliser et calibrer dans le développement d'hypothèses socio-économiques concernant le site même et jusqu'à un certain degré, son cadre (inter) régional. Comme nous l'avons exprimé plus haut, l'étude de la terre sigillée et l'image de l'approvisionnement en sigillée dans la région et dans les cités voisines ont fait l'objet de peu d'attention pour le moment, par conséquent nos analyses manquent d'un cadre de référence locale. Hormis ces restrictions, nous pensons pouvoir montrer que la courbe d'approvisionnement en sigillée est tout à fait caractéristique d'un site dont l'essor se situe dans le II $^{\mathrm{e}}$ siècle. Cette image générale n'est néanmoins ni fiable, ni valable sans les précisions et explications spécifiques, expliquées ci-dessous. De plus, la courbe d'approvisionnement de SteenePitgam reflète en premier instance les fluctuations et les tendances dans les centres de production mêmes et sur le marché inter-régional, donc sur le plan macroéconomique, comme cela fut démontré à Londres par Marsh (1981). Ces fluctuations pourraient même être des reflets d'un mouvement cyclique dans l'économie, similaire aux fameux cycles de Kondratieff (Going, 1992, p. 108-110).

Il est évident qu'une discussion concernant la date de la fondation de ce site ne peut être basée sur l'évaluation globale de tout l'assemblage céramique et numismatique, sans la combinaison avec des données contextuelles issues de fouilles. Toutefois, les données chronologiques, issues de l'étude de plus de 150 monnaies trouvées en surface (étude du Prof. R. Delmaire), sont similaires aux résultats de l'étude des sigillées. Pour le moment, l'assemblage de la sigillée ne permet pas l'élaboration d'une chronologie fine. Tout au plus peut-on attirer l'attention sur quelques tendances générales.
La quasi absence de sigillée précoce (pré-flavienne) et la présence minoritaire des productions du sud de la Gaule en général sont tout à fait contraires aux renseignements livrés par les prospections récentes de $\mathrm{Ph}$. Ducrocq sur une villa à Cassel (De Clercq et Deschieter, 1996b) où l'on a constaté que les statistiques sur l'approvisionnement en sigillée sont indicatrices de l'époque Claude-Flaviens. Cela est sans doute en relation avec la proximité immédiate du chef-lieu des Ménapiens et son rapide développement urbain et économique à cette époque. Le peu de matériel précoce à Steene-Pitgam pourrait donc être indicatif d'un développement plus restreint à l'époque pré-flavienne. Toutefois, nous pensons qu'il serait mieux de décrire ce phénomène - de façon plus prudente et objective - comme un approvisionnement en sigillée retardé, ce qui ne peut et ne doit nullement exclure une occupation antérieure (sous TibèreClaude par exemple). En tout cas, il est clair que l'importation de sigillée à Steene-Pitgam prend essor sous les règnes des empereurs Vespasien, Titus et surtout Domitien, ce qui est illustré autant par la quantité de sigillée décorée que par la vaisselle lisse. C'est donc à cette époque que le site prend sa place dans le réseau inter-régional de commerce (en sigillée), comme le font les autres sites « côtiers » dans la partie septentrionale de la cité des Ménapiens (Thoen, 1978, p. 113, 117, 194, 204 ; Van Beek, 1983, p. 3 ; TrimpeBurger, 1975, p. 135) et dans le sud-ouest des Pays Bas (Haalebos, 1993, p. 28). Cette constatation correspond bien à l'image qui a été esquissée pour l'Atrébatie au $\mathrm{I}^{\mathrm{er}}$ siècle (Tuffreau-Libre \& Jacques, 1985, p. 141-143).

Les productions du sud de la Gaule disparaissent dans les ensembles céramiques de la période 100-120, c'est-à-dire sous le règne de Trajan et au début du règne d'Hadrien. La place des ateliers méridionaux sur le marché est reprise par les officines du centre de la Gaule : Les Martres-de-Veyre (vers 100-120) et Lezoux (vers 120-200). Ce changement dans l'importation en sigillée a des conséquences quantitatives. Comme sur la majorité des sites, la courbe de SteenePitgam, tant pour la sigillée unie que pour les produits décorés, montre une baisse sensible à cette époque (fig. 4). Ce phénomène n'a rien à voir avec l'économie ou l'intensité de l'occupation au site de SteenePitgam même, mais est vraisemblablement lié aux problèmes de la distribution de la céramique sigillée. Evidemment les ateliers du centre n'étaient pas encore assez développés pour pouvoir s'emparer du marché ouvert par l'effondrement des ateliers du sud. Signalons qu'aucun potier de l'époque de Trajan ne figure parmi les pièces décorées du centre. Par contre, la présence de l'assiette remarquable Bet 47 semble 
dénoter un apport, sans doute limité, de vaisselle lisse du centre de la Gaule pendant ces premières décennies du II $^{\mathrm{c}}$ siècle. I e relais du marché par les ateliers du centre ne s'est donc pas passé avec aisance. Nous insistons sur le fait que ce phénomène n'est attribuable qu'à la situation inter-régionale et non pas à celle du site même, car on constate cette «baisse trajane » sur de nombreux sites. D'autre part, nous sommes convaincus que les productions tardives du sud de la Gaule ont continué à fonctionner dans les services de table pendant les deux premières décennies du II $^{\mathrm{e}}$ siècle, et qu'il ne s'agit donc pas de matériel résiduel comme il a été souvent supposé.

A partir de l'époque d'Hadrien, les ateliers du centre vont inonder le marché du nord-ouest de la Gaule de vases décorés et unis, pour finalement aboutir à un écoulement maximal vers 160-175 (fig. 4). Pendant les décennies suivantes, l'apport de sigillée connaîtra une chute sensible, malgré la proportion montante de bols décorés lexoviens. En effet, les décades qui suivront seront caractérisées par une diminution spectaculaire de l'approvisionnement en sigillée. Les raisons seront traitées plus loin.

En ce qui concerne le point optimum de la période antonine, différentes explications sont plausibles. Il faut d'abord noter que ce phénomène a été remarqué sur beaucoup de sites. Il peut être expliqué entre autres par un effondrement des prix (Galliou et Sanquer, 1979), peut-être en raison d'une production de masse et d'une concurrence parmi les différents potiers. D'autre part, la stabilité et la croissance économique pendant la pax romana ont abouti à une croissance du pouvoir d'achat pour la majorité de la population. La vulgarisation de la terre sigillée, de produit de luxe à produit de demi-luxe, serait ainsi liée à la romanisation et à l'élévation générale du niveau de vie dans la société (Raepsaet, 1987, p. 3 et Raepsaet-Charlier, 1988, p. 63). De plus, la plupart des sigillées de Steene-Pitgam de la période 100-175 à 200 provient des officines du centre de la Gaule $(87 \%)$, un phénomène quantitatif distinct des sites de la cité voisine des Morins (Raepsaet, 1987, tableau 2 et Delmaire, 1976). Cet élément serait à son tour lié à la nature même de l'approvisionnement, c'est-à-dire la commercialisation et la géographie commerciale de la vente en sigillée (Raepsaet, 1987, p. 5). L'apparition massive de sigillées du centre de la Gaule dans la région pourrait donc être due à l'embarquement de celles-ci à destination de la Britannia à travers les sites portuaires de la Manche (RaepsaetCharlier, 1988, p. 65). La géographie commerciale de la vente en sigillée s'exprimerait donc dans le fait que 80 à $90 \%$ des sigillées sur les sites d'Etaples, Amiens, Ardres et Boulogne proviennent des officines du centre pendant qu'à la même époque 60 à $95 \%$ des produits dans les sites les plus septentrionaux (Valkenburg, Arentsburg, Neuss, Bonn : avec un transport de sigillée par le Rhin vers la Britannia) sont originaires des ateliers de l'est (Raepsaet, 1987, p. 5). Nous émettons cependant quelques réserves quant à la fiabilité statistique dans la catégorie des sites de la Morinie comme indiqué chez Raepsaet-Charlier (1988, tableau 1). En plus, les auteurs indiquent qu'il existait peut-être un cabotage de l'embouchure du Rhin et de l'Escaut (avec de la sigillée de l'Est) vers les sites portuaires de la Manche, pour finalement traverser la mer du Nord (Raepsaet, 1987, p. 19). $S$ 'il existait réellement de vastes courants commerciaux de ce type, la céramique de l'est (surtout Rheinzabern) serait présente en plus grand nombre dans les sites de la Manche, ce qui n'est apparemment pas le cas. Néanmoins l'existence d'une géographie commerciale de la vente de sigillée n'est pas remise en question ici.

Une proportion importante de la sigillée décorée au deuxième siècle (fig. 6) à Steene-Pitgam provient des officines argonnaises (12\%). Dans la statistique totale des sigillées attribuées (fig. 5), les officines de l'Argonne représentent toujours $10 \%$, tandis que pour les ateliers du centre le chiffre monte à $75 \%$. Si nous ajoutions les Dr. 45 d'Argonne, le pourcentage total des produits argonnaises serait encore plus élevé. Le peu d'éléments de comparaison que nous possédons (Raepsaet, 1987, tableau 1, basé sur un nombre de tessons souvent limité) indiquent un maximum de $5 \%$ pour les produits argonnais (sites d'Ardres, Etaples, Thérouanne, Boulogne, Amiens, Arras, Bavay), ce qui est tout à fait contraire aux données de Steene-Pitgam. Cette prédominance persistera au $\mathrm{III}^{\mathrm{e}}$ siècle à SteenePitgam, plus spécifiquement en ce qui concerne la forme Dr. 45. Une explication pour cette vaste présence argonnaise pendant les deux siècles réside probablement dans les aires de production même et les réseaux de distribution qui mènent les produits jusqu'aux consommateurs. Si nous comparons l'approvisionnement en sigillée de la partie septentrionale de la civitas Menapiorum aux régions méridionales de la même cité et aux cités des Atrébates et Morins (Cassel, Steene-Pitgam et Ardres), nous constatons une prédominance des produits de Rheinzabern dans la première région (à partir de la deuxième moitié du $\mathrm{II}^{\mathrm{e}}$ siècle), et une quasi absence dans les autres cités. Précisons que les sites côtiers — donc souvent des sites sauniers - de la partie septentrionale de la cité des Ménapiens (Thoen, 1978, p. 161-163 et fig. 55) sont bien pourvus de produits de Rheinzabern, ce qui est contraire aux données de Steene-Pitgam et aux sites mentionnés par Raepsaet (1987, tableau 1). Cette 
image est bien illustrée par des sites comme Oudenburg (Creus, 1975) et Zeebrugge (Hollevoet, 1989 , p. 41) où la prépondérance des produits de Rheinzabern est écrasante comparée à la terre sigillée de l'Argonne.

En outre, nous ne trouvons aucun élément suffisamment convainquant pour soutenir la thèse que la sigillée argonnaise serait un fret de retour sur les chariots des commerçants en sel ou salaisons, venant de la côte et approvisionnant les sites militaires et civils à l'intérieur (comme proposé par van Beek, 1983, p. 11). Nous pensons que ces divergences s'expliquent plutôt par les chemins de transport, donc par la géographie commerciale. Si les produits de Rheinzabern ont connu leur diffusion en bateau sur le Rhin jusqu'au Helinium (et puis au-delà par la mer du Nord vers la Britannia), une distribution vers l'Escaut, la Durme et la Lys explique l'importation de ceux-ci vers la partie nord de la civitas Menapiorum. Quant aux régions plus méridionales, un approvisionnement par le réseau routier bien développé sur l'axe nord-ouest / sud-est peut expliquer la présence significative de produits d'Argonne à Steene-Pitgam. La raison pour laquelle les autres sites ne sont pas compris dans ce mouvement reste pour le moment difficile à expliquer. Même si le site de Steene-Pitgam n'a pas connu d'occupation intense au III $^{\mathrm{e}}$ siècle (une des raisons possibles pour le manque de produits de Rheinzabern), les produits de Rheinzabern d'époque antonine manquent manifestement à Steene-Pitgam.

Contrairement à la situation du II ${ }^{\mathrm{e}}$ siècle, l'importation des vases moulés ornés au III $^{\mathrm{c}}$ siècle n'est guère attestée à Steene-Pitgam. Cette baisse est due à différentes raisons de genre macro- et micro-économiques, car on aperçoit ce phénomène ailleurs qu'à SteenePitgam (Bird, 1986 pour Londres). Parmi les explications possibles, on peut citer la récession économique succédant aux troubles politiques à la fin du $\mathrm{II}^{\mathrm{e}}$ siècle, mettant fin aux années de luxe el de vaste circulation monétaire. Nous nous référons également aux problèmes militaires, vers 170-175, lors des attaques des Chauques dans le nord de la Gaule. Une diminution de la proportion de la sigillée décorée dans l'ensemble de la terre sigillée - cause purement typologique est une des autres raisons possibles. Cependant, nous constatons que, dans le répertoire formel, les pièces typiques du III ${ }^{\mathrm{C}}$ siècle (des Dr. 31 de l'est, Dr. 32, les formes de la typologie de Ludowici) manquent dans l'ensemble de Steene-Pitgam, si l'on excepte la présence de nombreux mortiers Dr. 45 (supra). Signalons enfin la faiblc part que représentent les sigillées de Trèves. Comme pour la sigillée de Rheinzabern, les bols décorés trévires du II $^{\mathfrak{c}}$ siècle font complètement défaut et on ne dénombre que deux Dr. 37 de la première moitié du $\mathrm{II}^{\mathrm{e}}$ siècle.

En tout cas, dès le dernier quart du $\mathrm{II}^{\mathrm{e}}$ et surtout au début $\mathrm{du}$ III $^{\mathrm{e}}$ siècle, nous constatons une diminution spectaculaire de l'approvisionnement en sigillée. Ce phénomène est d'une part sans doute lié aux facteurs économiques sur un plan inter-régional mais pourrait d'autre part être imputable aux activités (commerciales) et aux événements sur le site de Steene-Pitgam même. Nous pensons dans ce cas aux invasions des tribus germaniques maritimes. A partir de la deuxième moitié du III $^{\mathrm{e}}$ siècle, nous ne possédons aucun tesson de sigillée, malgré le fait qu'une occupation militaire au Bas-Empire, dans le cadre d'une défense littorale, a été supposée à Steene (Cools, 1985, p. 19, fig. 2; Roumegoux \& Termote, 1993, fig. 53). Peut-être d'autres sites se révéleront-ils dans un prochain futur.

Cette étude, fondée sur un matériel de surface, n'est donc qu'une première tentative pour analyser et mieux comprendre l'approvisionnement en sigillée d'un site romain important à un emplacement géographique et pédologique extraordinaire. Nous avons essayé d'attribuer les pièces à des styles de potiers et à des ateliers spécifiques, afin d'aboutir à des statistiques, utilisées pour le développement d'hypothèses concernant l'économie du site. De même, nous avons cherché à placer les données dans un cadre de référence inter-régional. Nous attendons de nouvelles fouilles et de nouveaux contextes, bien datés et bien évalués dans le Nord et dans le Pas-de-Calais, pour mieux comprendre l'image de la sigillée dans cette région et sur le site de Steene-Pitgam.

Wim De ClercQ et Johan DeschIETER avec la collaboration de Joanna BIRD et Brenda DICKINSON

Wim DE ClekrcQ, Rostraat 12, 9850 Hansbeke, Belgique. Johan Deschieter. Guido Gezellestraat, 21, 8550 Zwevegem, Belgique. Joanna BIRI, 14 Kings Road, Guildford GU1 4JW, Grande-Bretagne. Brenda Dickinson, University of Leeds. School of Classics, Leeds LS2 9JT, Grande-Bretagne.

\section{CATALOgUE}

\section{A. La sigillée lisse}

\section{Sud de la Gaule}

1. Dr. 24-25 (S1) : pré-flavien

2. Ritt. 9 (S10) : pré-flavien

3. Dr. $15 / 17$ (S1) : pré-flavien

4. Dr. $15 / 17$ (S8) : pré-flavien

5. Dr. $15 / 17$ ou 18 (S2) : pré-flavien 10

6. Dr. 18 (S1) : flavien

7. Dr. 36 (S8) : flavien

8. O\&P. LIV, 8-9 / Vernhet (1976) El (S4) : flavien 
9. Dr 27 (S3) : SECVNDI : flavien

10. Curle 11 (SI) : flavien

11. Vernhet (1976) B2 (S) : flavien

\section{Centre de la Gaule.}

12. Bet 47 (S3)

13. Dr. $18 / 31$ (S2) : Nicephor II de Lezoux, vers $140-155$

14. Dr. $18 / 31$ ou 31 (S2) : Antonin

15. Dr. $18 / 31$ ou 31 (S2) : Antonin

16. Dr. 31 (S9) : ]MVS : Antonin tardif

17. Dr. 31 (S1) : Mainacnus de Lubié et de Lezoux, vers 160-200

18. Walt.79/80 (S1) : Antonin tardif

19. Dr. 36 (S2) : Antonin

20. Dr. 33 (S1) : Saturninus II de Lezoux, vers $160-180$

21. Dr. 33 (S7): Genialis IV de Lezoux, vers $160-190$

22. Dr. 33 (S1) : Malledo de Lezoux, vers $150-180$

23. Dr. 33 (S1) marque anépigraphique : Antonin

24. Dr. 27 (S1) : Antonin

25. Dr. 46 (S1) : Antonin

26. O\&P, LV, 13 (S2) : Antonin

27. Dr. 38 (S3) : Antonin

28. Dr. 38 (S8) : Antonin

\section{Est de la Gaule}

29. Dr. 33 (S1) : Antonin-Troisième siècle

30. Dr. $40(\mathrm{~S} 1+\mathrm{S} 2)$ : Troisième siècle

31. Dr. 30R (S1) : Antonin

32. Dr. 31 (S8) : Antonin tardif

33. Dr. $43(\mathrm{~S} 1)$ : Antonin-Troisième siècle

34. Dr. 44 (S2) : Antonin-Troisième siècle

35. Dr. $45(\mathrm{~S} 2)$ : Antonin-Troisième siècle

36. Dr. 45 (S3) : Antonin-Troisième siècle. Tête de lion à provenance incertain (Rheinzabern?)

37. Dr. 45 (S1) : Fin deuxième-début troisième siècle : Argonne : Avocourt-Forêt-de-Hesse, type b

38. Dr. 45 (S1) : Fin deuxième siècle : Argonne : Les-AllieuxClairière ou Avocourt-Forêt-de-Hesse, type a

39. Canthare Lud. VMd ou VMe, deuxième moitié II s., première moitié III $\mathrm{s}$.

\section{B. Les vases moulés ornés}

\section{Les produits du sud de la Gaule}

\section{a) Les produits de l'époque pré-flavienne (Claude-} Néron)

1. (Inv. S3) Dr. 29. Fragment de bord avec décoration d'une bande de lignes. Sud de la Gaule, période Néron-Vespasien.

2. (Inv. S2) Dr. 29. Fragment de panse décorée de zones séparées par deux cordons perlés. La zone inférieure est ornée d'une succession d'un médaillon et d'un sautoir avec trifol comme Knorr 1919 : Textbild 12,6. Le style et la composition de cette frise sont caractéristiques pour la période Néron-début Vespasien.

3. (Inv. S10) Dr. 30. Fragment de paroi. Décor de rinceaux avec feuille de fougère utilisée par Masclus (Knorr 1919, Taf. 52, nr. 15 et 19), Aquitanus (Knorr 1919, Taf. 8, 4), Labio (Knorr 1952, Taf. 32) et Licinius (Knorr 1952, Taf. 34, B), grande et petite feuille pentafoliée comme à Velzeke (V19) et feuille palmée (Knorr 1919, Taf 95, D). Guirlande comme à Valkenburg (Abb. 5, 15 : Masclus), Velzeke (V9) et La Graufesenque (Hermet, P1. $69,14)$. Style de Masclus de La Graufesenque, ou un de ses contemporains. Claude-Néron.

\section{b) Les produits flaviens (Vespasien, Titus, Domitien)}

4. (Inv. S3) Dr. 37. Fragment de bord. Ove à coeur central et orle double. Dard à druite avec pendentif trifolié, rentrant à gauche comme à Iuvavum (Taf. 18, 6-12) : Severus; Margidunum (PI. XI, 2, 33) : Severus. Ove Hofmann (1988) D3, connu à Banassac et à La Graufesenque. Fragment de rinceaux. Severus de La Graufesenque : Vespasien-Domitien.
5. (Inv. S1) Dr. 37. Bas de panse. Décor de métopes séparées par des lignes ondulées. Sautoir. Rinceau de feuille cordiforme. Le décor est terminé par une frise de feuilles trifoliées comme Knorr 1919, Textbild 12; ces feuilles figurent dans l'oeuvre des potiers flaviens comme Iucundus, Vitalis, $L$. et Mercato(r). La métope à gauche a vraisemblablement contenu un médaillon à deux ou trois bordures unies, encadré par quatre rinceaux aux feuilles cordiformes. Ce schéma décoratif est un élément caractéristique des Dr. 37 de l'époque Vespasien-Domitien comme le montrent des exemples à Verulamium (I, fig. 90) et à Pompei (Atkinson 1914, $\mathrm{n}^{\circ}$ 55, 60 et 73-74). Sud de la Gaule : Vespasien-Domitien.

6. (Inv. S2) Dr. 37. Fragment de paroi. Décor en style libre Pêcheur Osw. 952 (= Déch. $557=$ Hermet $200=$ Knorr 1919 , Taf. 34, 9 : Germanus, aussi à Sorviodurum : Taf. 1, 7). Végétation Hermet, Taf. 101, 37 et ondes, comme à Sorviodurum : Taf. 1,6 et 7. Arbre, également comme à Sorviodurum : Taf. 1, 6. Style de Germanus III de La Graufesenque, Flavien.

7. (Inv. S2) Dr. 37. Fragment de paroi. Décor de métopes au moyen de lignes perlées. Frise intérieure d'éléments ou de feuilles trifoliées (Knorr 1919, Textbild 12, 5 et Taf. 57, 12). Fragment d'une estampille étroite : ] $O$, placée verticalement dans une métope : Mercato. Voir aussi : Knorr 1919, taf. 57 D. A droite, une partie du motif Knorr 1919, Taf. 57, 20. Mercato de La Graufesenque, Domitien.

8. (Inv. S1 vers S9) Dr. 37. Fragment de paroi. Décor de rinceaux, avec grande feuille emplumée (Déch. $1169=$ Hermet 8,20 ). Ce motif est fréquemment utilisé dans l'oeuvre de Calus de La Graufesenque (Knorr, 1952, p. X, 2 fig.); mais est surtout associé aux produits de Mercator : (Verulamium III, p. 187) et Mees 1995 (Taf. 128,1-2 et taf. 133, let 3). Oie du Nil à droite : Hermet 1934, Pl. 28, 68. Mercato ou Calus de La Graufesenque : Flavien tardif.

9. (Inv. S8-1996) Dr. 37. Fragment de paroi. Ove mal venu et peu lisible. Dard à gauche, à tête d'étoile comme à Caerleon (fig. 9 : 14 et 15, fig. $10: 16$ ) : Frontinus, Paullus et Mercato. Mees 1995, Taf. 18h 1-4 et Taf. 19, 1-8: décorateur Calvinus, Taf. 1517 : décorateur Calvus et Taf. 165, 4-9 : estampillé Paullus; Alchester : 189 : Frontinus. Décor de métopes au moyen de petites perles carrées; étoiles aux jonctions. A gauche : bestiarius Osw. 1102 comme montré dans Hofmann (1988; p. 142, 76 et Pl. 15, 120) : Biragillus. Si l'attribution de notre tesson à un potier spécifique doit rester incertain, la datation par contre se trouve sans doute vers la dernière vingtaine d'années du premier siècle après J.-C. Sud de la Gaule (La Graufesenque ou Banassac), Domitien.

10. (Inv. S9) Dr. 37. Fragment de paroi. Zone avec pointes de flèche dans un décor à grands rinceaux. Au-dessous de la ligne ondulée, probablement un lion (?). Le schéma décoratif se rapproche bien de celui utilisé par des potiers de l'époque flavienne comme par exemple Mercato. Pontus et $M$. Crestio. Sud dc la Gaulc, Flavien.

\section{Les produits du centre de la Gaule}

\section{a) Les produits de l'époque Hadrien-Antonin-pré-} coce (117-145).

11. (Inv. S1) Dr. 37. Fragment de paroi. Décor en style libre ou de métopes. Frise d'oves à coeur filiforme et orle double. Dard à droite, à tête globuleuse se terminant dans un petit pendentif : Rogers B113: Avitus. La frise d'oves est bordée d'une ligne à petites perles. Frise de trifols à gauche (similaire aux frises de trifols flaviens du sud de la Gaule) comme $P G C$ (Pl. 64, 17 en position verticale) : Avitus. Les frises de bifols ou trifols sont des éléments décoratifs souvent rencontrés dans l'oeuvre d'Avitus-Vegetus comme par exemple : PGC (Pl. 63, 11,13) : Avitus-Vegetus. Style d'Avitus-Vegetus, de Lezoux, vers 120$150(P G C$ : p. 136).

12. (Inv. S2) Dr. 37. Fragment de paroi. Décor en métopes constituées de lignes tremblées. Décor probablement en forme de croix de St.-André, formées par des cordons tremblés. A 
rapprocher au style des potiers de l'époque Trajan-HadrienAntonins (précoce), comme par exemple : Avitus-Vegetus, Geminus, Potter $X-5$ et le groupe de potiers autour de Quintilianus (PGC : Pl. 62 à 71 ). La combinaison de lignes tremblées et de petits cercles se trouve entre autres dans le style de Quintilianus ( $P G C:$ Pl. 70, 18). A gauche, un pygmée nu, armé d'une lance. Ce motif est employé dans les décors des bols du centre, autant que de l'est de la Gaule (La Madeleine. Rheinzabern). Dans le matériel du centre de la Gaule, ce motif apparaît dans les productions précoces comme celles de Ianuaris I-Quintilianus (PGC : Pl. 69, 14). Au milieu : pilastre de bifols entre deux lignes tremblées. Bifol Rogers G282: CGP (fig. 14,2, Pl. 62, 1, 3: Pl. 64, 22) 0: AvitusVegetus. A droite : Silène, dansant vers la droite : Osw. 591, et aussi : $P G C(\mathrm{Pl}, 64,23)$ : Quintilianus. La pâte et la disposition générale de l'espace sont caractéristiques pour les produits de la première moitié du deuxième siècle. Le pilastre bifolié, les lignes tremblées et la croix de St.-André sont également des éléments distinctifs de cette période, et plus spécifiquement dans l'oeuvre d'Avitus-Vegetus. Le petit guerrier (plutôt style de Quintilianus) nous indique cependant la ressemblance artistique des potiers de cette époque. Cette similitude a déjà été constatée ailleurs (PGC, p. 134). Groupe de Quintilianus ou Avitus-Vegetus, vers 120-150. (CGP: p. 136 \& 147).

13. (Inv. S2) Dr.37. Fragment de paroi. Estampillage partiel des feuilles et des sabots d'un animal comme $P G C$ : Pl. 58,5 : Sacer. La double bordure en dessous de la décoration figure dans $P G C$ (Pl. 82,1 et 6 à Pl. 83, 9 et à Pl. 84, 17). Style de Sacer de Lezoux, vers 120-140/145.

14. (Inv. S2) Bige : variante des figures Osw. 117 et $117 \mathrm{~A}$. Ce motif figure sur des Dr. 37 trouvés à Asciburgium (2, Taf. 3,17 ), attribué à Quintilianus-Ianuarius-Paterclus, et à Dover (335 et 341), attribué aux styles de Cinnamus et Albucius. Le motif sous le bige pourrait être le même que celui du bol d'Asciburgium (2, Taf. 3, 17). Probablement vers 125-150.

15. (Inv. S3) Dr. 37. Fragment de bord. Décor libre de végétation. Ove à coeur large avec orle double, dard cordé à tête assez globuleuse à gauche : Rogers B35:X-6.PGC:Pl. 18,1:X-6; Tongeren : Pl. V, $47: X-6$. Ligne tremblée comme $C G P: \mathrm{Pl} .74$, 2, 3, $6: X-6$. Rinceaux avec feuille pentafoliée et nervurée Rogers $\mathrm{H} 73: X-6$ comme $P G C$ : fig. 18,9 et Pl. 74,2:X-6. Sans doute style du potier $X-6$ : vers $125-150$ (PGC, p. 194) ou 125-145 (Rogers, 1977, 248).

16. (Inv. S3) Dr. 37. Fragment de paroi. Décor libre de végétation. Rinceaux avec feuille pentafoliée et nervurée Rogers $\mathrm{H} 73: X$ 6 comme CGP : fig. 18,9 et Pl. 74,2:X-6. $X-6$. Vue le décor, la technique et le finissage, ce tesson provient peut-être du même bol que S3 / S7.

17. (Inv. S7) Dr. 37. Fragment de paroi. Décor libre de végétation. Ove à coeur large avec orle double, dard cordé à gauche à tête globuleuse : Rogers B35 : X-6. PGC : Pl. 18,1 : X-6 ; Tongeren : $\mathrm{Pl} . \mathrm{V}, 47: X-6$. Ligne tremblée comme $P G C: \mathrm{Pl} .74$. 2, 3, $6: X-6$. Rinceaux avec feuille pentafoliée et nervurée Rogers II73: $X-6$ comme $P G C:$ fig. 18,9 et Pl. $74,2: X-6$. Sans doute style du potier $X-6$, peut-être provenant du même moule que $S 3-1,2$

18. (Inv. S2) Dr. 37. Fragment de paroi. Décor en style libre (?). Ove à coeur large avec orle double, dard cordé à tête assez globuleuse à gauche : Rogers $\mathrm{B} 35: X-6 . P G C: \mathrm{Pl} .18,1: X-6$; Tongeren : $\mathrm{Pl}$. V, $47: X-6$. Tortillon ou feuille nervurée (indéterminé). Style de $X-6$.

19. (Inv. S2) Dr. 37. Bas de panse d'un Dr. 37. Décor en style libre, probablement avec des grands rinceaux comme dans le style du potier Attianus (PGC : Pl. 85, 6 et Pl. 86, 18; Corbridge: fig. 105,26: ATTIANI). Surtout la disposition de grands cercles (CGP : fig. 23, 9 Attianus) en bas de la frise est un élément qui se rapproche de l'oeuvre d'Attianus. Style d'Attianus (vers 120-140/145)

20. (Inv. S7) Dr. 37. Fragment de paroi. Sphinx tourné à droite Osw. 856. Deux diclytrae : Rogers G141. Acanthe renversé ? Le sphinx n'est attesté (selon l'index de F. Oswald) que dans 'oeuvre d'Attianus et d'Acaunissa. Style d'Attianus ou Accaunissa. (vers 120-140/145).
21. (Inv. S4) Dr. 37. Fragment de paroi. Métopes étroites et peu profondes séparées par des cordons nettement serrés et par des lignes perlées. Le motif à gauche est probablement une colonne horizontale au chapiteau perlé; le fût torse est encadré. (Pour le fût : Rogers : P42 qui est malheureusement incomplet et ne porte aucune trace de chapiteau). Le motif central est probablement un cordon simple (comme Rogers U153) qui s'est formé lors du façonnage. Un motif comparable figure sur un bol moulé de Tittius, trouvé à Braughing (fig. 42,3 ) et sur un bol dans le style de Pugnus (CGP, PI.154, 17). Le motif à droite est vraisemblablement une feuille emplumée (cf. Rogers : J136) sens dessus-dessous. Malgré les dimensions des métopes et des lignes perlées relativement fines - éléments souvent rencontrés sur des bols anonymes de l'époque d'Hadrien -, la technique et l'épaisseur de la paroi sont cependant plus caractéristiques pour les produits plus tardifs; une date dans la période hadrienne-antonine précoce nous semble donc plus acceptable.

22. (Inv. S1) Dr. 37. Fragment de bord. Décor libre. Frise d'oves à coeur avec orle double, dard central à tête globuleuse et perforée : $P G C$ (Pl. 90,1 et 2): Birrantus. Cordon de perles fines. Pygmée combattant à gauche : Osw. 699a : Birrantus, Butrio; $P G C:$ Pl. 90,1 et $2:$ Birrantus. Astragale comme PGC : Pl. 90,1 : Birrantus. A gauche : homme nu debout : variante $O s w$. 681; PGC: Pl. 150,39: Doeccus. A droite : homme nu debout à gauche (indéterminé). A l'extrême droite : pygmée $O s w$. 658c : Birrantus. En dessous : motif indéterminé (végétation?).

Les deux hommes nus ne sont pas repris dans l'index de Félix Oswald. Malgré la datation indiscutable du tesson en question, on peut constater que l'une de ces figures masculines figure même dans l'oeuvre de Doeccus (deuxième moitié deuxième siècle, $P G C$, Pl. 150,31). La majorité des motifs datables. autant que la pâte et la disposition générale de l'espace indiquent cependant une datation dans l'époque Trajan-Hadrien. Le cordon perlé au moyen de perles fines est en effet souvent rencontré chez des potiers de cette époque et presque pas chez les produits connus de l'époque d'Antonin ( $P G C$, p. 171). Malgré la domination du style de métopes dans l'œuvre de Birrantus, la détermination et la combinaison de l'ove, de l'astragale et des deux pygmées sur le tesson de Steene-Pitgam sont des éléments suffisamment distinctifs pour l'attribuer au potier Birrantus, vers $125-150$.

23. (Inv. S2) Dr. 37. Fragment de bord. Ove à coeur et à orle double. Dard accolé à droite, à tête globuleuse comme à Saalburg, Taf. 28,17a. Décor de métopes séparées par des cordons ondulés. Astragale Rogers R63. Petits cercles. Harpie Osw. 863A. L'ove et le style général de ce fragment ressemblent fort à deux tessons provenant de Saalburg (Taf. 28, 17a et $17 \mathrm{~b}$ ). Rogers les attribue au potier Secundinus I de Lezoux (Rogers, 1974, p. 168, R63). Style de Secundinus I de Lezoux. Hadrien.

24. (Inv. S1) Triton brandissant une massue. Ce remarquable triton, finement achevé et à qucuc finc n'cst pas repris dans l'index d'Oswald. Par conséquent nous sommes dans l'impossibilité d'attribuer cette pièce à quelque potier. Néanmoins, la bonne qualité du finissage ainsi que la paroi mince plaident en faveur d'une date dans la période d'Hadrien. Centre de la Gaule, époque Hadrien - Antonin précoce.

\section{b) Antonin précoce à Antonin moyen (145-170).}

25. (Inv. S1) Dr. 37. Fragment de paroi. Décor de métopes séparées par des lignes ondulées. Apollon nu, assis : Osw. 83. Rinceau élaboré : Rogers M50. Ce motif, ainsi que la disposition des métopes au moyen de lignes ondulées et des rosettes sont souvent attestés dans le répertoire de l'association CriciroAventinus $I$ (PGC : fig. 51,2). Accumulation de cercles, se retrouve dans l'oeuvre du potier Criciro, soit dans une position verticale $(P G C:$ Pl. 172, 1 ; Ovilava : Taf. 59,4$)$ soit disposćc horizontalement (PGC: Pl. 117,2; Ovilava : Taf. 59,3). L'Apollon figure également sur un bol signé de Criciro (PGC: Pl. 172,1). Style du groupe Criciro-Aventinus I (vers 130-150). 
26. (Inv. S1) Dr. 37. Fragment de paroi. Ove à coeur filiforme, orle double et dard perlé à gauche. confondu avec l'orle extérieur Le dard est composé de 6 perles carrées et d'une rosette à l'extrémité : Rogers B 47 : Criciro; PGC (Pl. 117, 1, 3, 8; Pl. 118 , 12, 13) : Criciro. Style de Ćriciro. (130-150).

27. (Inv. S3. Dr. 37. Bien que la décoration semble s'arrêter sous la ligne perlée horizontale (c'est-à-dire sans ove), le finissage lisse à l'intérieur indique qu'il s'agit bien d'un très petit $\mathrm{Dr}$. 37 , et non d'un vase moulé. La technique est sans doute celle de Lezoux. Le motif à droite est une colonne torse et encadrée, probablement Rogers P42, flanquée de deux cordons aux perles rectangulaires. Cette colonne se rencontre sur deux bols récemment découverts à Sandy (Angleterre; fouilles non publiées) et à Exeter (fig. 16,86) dans le style d'un potier anonyme. Le petit oiseau représente une version plus petite du type $O s w .2295$ A; il s'agit peut-être d'un surmoulage. Il semble que la petite figure masculine, qu'on peut identifier comme Hercule Osw. 750, soit posé sur un socle, ce qui n'est pas le cas avec $O s w .750$. L'identification du motif dans le double médaillon reste difficile ; peut-être a-t-il été endommagé lors du finissage. Probablement vers 135-155.

28. (Inv. S1) Dr.30, trop cuit. Masque, légèrement abîmé sur le tesson : $P G C, P 1.141,12$ : « le potier au petit $S$ ». La figure pourrait être le danseur nu à $\mathrm{Pl} .142,17$. Pour la petite feuille, le motif en S et le cordon perlé : Pl. 142, 29 : Satus-Cettus. La petite feuille sans tige est comparable à Terrisse, 1968 Pl. XXII, 232, et le médaillon représente probablement le cercle extérieur du petit médaillon double à PI. XXII, 274-275 La feuille à côté du masque ne connaît aucun parallèle dans Rogers 1974 : le style du motif J20 de Rogers semble comparable mais la partie centrale est plus grande et plus arrondie. Satus-Cettus de Les Martres-de-Veyre, vers 135-160 (Hartley, 1972,34 et Rogers, 1977, 248).

29. (Inv. S7) Dr. 30. Fragment de paroi. Ove mal venue, il s'agit très vraisemblablement d'une ove à orle simple, probablement celle du potier Divixtus : $P G C$; fig. 33 : ove 4 . Décor de métopes au moyen de lignes perlées. Dans un médaillon à double bordure unie : lièvre, assis à gauche : Osw. 2116 Caryatide Osw. 1199. Trophée Rogers Q10. Les motifs et le style sont identiques à un Dr. 30, trouvé à $U s k$ (fig. 103, 273) : Divixtus. Style de Divixtus, vers 140-160 (PGC: p. 250).

30. (Inv. S2) Dr. 37. Fragment de paroi. Décor de métopes au moyen de lignes perlées, timbrées de petits cercles à la jonction des autres cordons perlés. A droite et à gauche : médaillon à double bordure unie. Positionnée au centre de deux pilastres perlés : caryatide Osw. 1207a; PGC (Pl. 115, 7; 116, 8-15) : Divixtus; Vendeuil, 360 : Divixtus; Vervoz, Pl. J, 4-5 avec estampille intradécorative : DIVIXT. Le style de métopes aux perles légèrement carrées et aux petits cercles aux jonctions, est un élément distinctif de Divixtus : $C G P(\mathrm{Pl} .116,10,11,14$, 15, 17); Vervoz, Pl. J, 4-5 avec estampille intradécorative : DIVIXT. Dans le registre inférieur : chien à gauche, peut-être Osw. 2007a; Vervoz, PI. J, 4-5 avec estampille intradécorative : DIVIXT. Produit distinct du potier Divixtus, vers 140-160. Bol à décor identique à Vervoz ( $\mathrm{Pl} . \mathrm{J}, 4-5)$.

31. (Inv. S7) Dr. 37. Bas de panse, trace de réparation. Décor de métopes, constituées de lignes ondulées. Panthère galopant vers la droite : Osw. 1518. Probablement un fragment du motif de vigne Rogers M2 et d'un petit médaillon (?). La combinaison des cordons ondulés avec des rosettes pourrait suggérer des affinités stylistiques avec l'oeuvre du potier Tetturo (PGC:Pl. 131, 1 et 3; Wacher 1969, fig. 49, 46). Par contre, ces lignes tremblées nous semblent trop fines et étroites ce qui suscitent plutôt des associations au style de Divixtus (Ovilava, Taf. 57 7). Probablement vers 140-160.

\section{CINNAMUS-CERIALIS}

a) style à ove 3 : 135-145 (Rogers, 1972, p. 322-326)

32. (Inv. S1) Dr. 37. Fragment de paroi avec percement (réparation). Décor de métopes. Frise d'oves à coeur filiforme abîmé, avec orle double. Dard mal venu à droite, constitué de six perles carénées : Simpson et Rogers ove 3B : employé par Cinnamus, Cerialis, Borillus, Annunus II: fig. 2, 13 : signé par Cerialis. Plumes comme à Ovilava: Taf. 70, 2, 3 : Cinnamus: Simpson et Rogers, fig. 2,13: Cinnamus-Cerialis. Vulcanus, courbé à droite : motif souvent utilisé dans l'oeuvre de Cinnamus et des potiers associés : Osw.13: PGC : Pl. 164,3 : signé en cursive Cerialis et estampillé Cinnamus, Pl. 154,14,16: Pugnus; Ovilava : Taf. 65,7: Cinnamus.

33. (Inv. S7) Dr. 37. Fragment de paroi. Décor de métopes avec feston. Frise d'oves à coeur filiforme abîmé, avec orle double. Dard mal venu à droite, constitué de six perles carrées Simpson et Rogers ove 3B : Cinnamus, Cerialis, Borillus, Annunus II; fig. 2. 13 : signé par Cerialis. Feston lisse avec feuille Rogers $\mathrm{J} 93$ à droite : Cinnamus. Style de CinnamusCerialis. Une pièce similaire a été publiée par Simpson et Rogers (fig. 2, 13). Même moule?

34. (Inv. S1) Dr. 37. Fragment de paroi. Décor - peut-être - en style libre. Plume comme à Ovilava: Taf. 70, 2-3: Cinnamus. Simpson et Rogers fig. 2,13 : Cinnamus-Cerialis. PGC : fig. 47,17 : Cinnamus. Patte avant d'un animal bondissant (indéterminé). Style de Cinnamus-Cerialis.

\section{b) Style de Cinnamus, autres oves : ca 135-170}

35. (Inv. S2) Dr. 37, brûlé. Fragment de paroi. Ove 5 de Cinnamus (CGP, fig. 47,5) : ove à coeur, orle double et dard à droite : Rogers B 182 : Cinnamus, CGP : fig. 47,3 : Cinnamus. Décor de métopes constitué de lignes perlées. Homme drapé : Osw. 905; Margidunum : Pl. XLII, 1 : Cinnamus; Ovilava : Taf. 76,3 : Cinnamus; PGC : Pl. 157,8 et Pl. 158, $16:$ Cinnamus. Style de Cinnamus.

36. (Inv. S1) Dr. 37. Fragment de paroi. Frise d'oves mal venues probablement ove Rogers B143 ou 144 du potier Cinnamus. Décor de métopes constituées de cordons perlés. Femme drapée Osw. 931; Ovilava : Taf : 45, 2 : Censorinus. Probablement style de Cinnamus.

37. (Inv. S1-1996) Dr. 37. Fragment de paroi. Ove à coeur central et orle double, dard à gauche, légèrement rentrant et à tête globuleuse : ove Rogers B231 : Cinnamus; $P G C$, fig. 47, 2 : Cinnamus. Cavalier, variant d'Osw. 245. Style de Cinnamus.

38. (Inv. S1) Dr. 37. Fragment de paroi. Décor de métopes constituées de lignes perlées. Masque gisant, variante Osw. 1214. Dans un médaillon à double bordure unie : danseur : Osw. 348; aussi à Newstead (sur un bol signé CINNAMI) et aux marais de Medway (fig. 3, 13). Au-dessus : deux torsades : $P G C$, fig. 47, 35: Cinnamus. Homme nu, marchant à droite : Osw. 688; Au-dessus : demi-trophée : $P G C$ : fig. 47, 39 : Cinnamus; Ovilava: Taf. 66,2 : Cinnamus. Style de Cinnamus.

39. (Inv. S2) Dr. 37. Fragment de paroi. Décor mal venu et abîmé. Décor de métopes, constituées de lignes perlées. Médaillon à double bordure unie. Victoire : Osw. 819A : Cinnamus; $P G C$ : Pl. 161 : 55 et Pl. 160, 46 : Cinnamus. Trophée Rogers L 11 ; $P G C$ : fig. 47,39; Pl. 160,41,45 : Cinnamus; Ovilava : Taf. 69,4 : Cinnamus. Style de Cinnamus.

40. (Inv. S3) Dr. 37. Fragment de paroi. Décor de métopes. Astragale Rogers R70 : Cinnamus, X-6; PGC : fig. 47,41 Cinnamus. Cordon perlé, terminant dans un petit cercle Ovilava : Taf. 74, 3-5,7 : Cinnamus. Style de Cinnamus.

41. (Inv. S7) Dr. 37. Fragment de paroi. Dans un médaillon à double bordure unie : lièvre, assis à droite : Osw. 2061. Le médaillon presque circulaire, en combinaison avec la bordure de perles carrées, pourrait indiquer des associations au style de Cinnamus. Probablement style de Cinnamus.

42. (Inv. S7) Dr. 37. Fragment de paroi. Décor en style libre. Cerf ou daim courant à gauche : Osw. 1781 : Cinnamus : ce motif apparaît fréquemment dans l'oeuvre signée de Cinnamus (CGP, Pl. 163, 66). Style de Cinnamus.

43. (Inv. S1) Dr. 37. Fragment de paroi. Décor libre avec scène de chasse. Panthère à droite : Osw. 1520; Ovilava : Taf. 80,6 : Cinnamus, CGP : Pl. 163,67 : Cinnamus et quelques potiers associés comme Cerialis et Paullus; ours à gauche : Osw. 1627; CGP : Pl. 163, 66, 68: Cinnamus: mais aussi Pl. 165,1: Paullus; Ovilava: Taf. 79, 4 : Cinnamus. Autre ours à gauche : 
variant Osw. 1631 (plus grand que S1-4) et aussi : PGC : Pl. 165,3 : Paullus; Ovilava : Taf. 77,2 et 78,3: Cinnamus. Cerf à gauche : Osw. 1814 (plus grand que S1-4). Demie feuille dentelée : CGP : Pl. 165,3: Paullus (en association avec panthère et ours); Pl. 160, 38 et 162,58 : Cinnamus. Pointe de lance? A rapprocher du style de Cinnamus ou Paullus.

44. (Inv. S1) Dr. 30. Fragment de paroi. Décor de métopes avec feston. Feston avec oiseau à droite : PGC : PI. 159,26 Cinnamus. Dans le registre inférieur : homme nu à droite : PGC : Pl. 159,26: Cinnamus : Etaples : Pl. 24. 3 : Cinnamus. Style de Cinnamus.

45. (Inv. S3) Dr. 30. Fragment de paroi. Décor en style libre. Homme nu, marchant à droite : Osw. 688;PGC: Pl. $159: 26$ : Cinnamus. Daim bondissant à droite : Osw. 1720; PGC : PI. 158, 21 et Pl. 163,66: Cinnamus. Cerf couché : Osw.1704; PGC : Pl. 158, 21, Pl. $159: 25$ et Pl. $163: 66$ : Cinnamus. Sanglier galopant à droite : Osw. 1638 : Cinnamus; PGC. Pl. 163,66 : Cinnamus. Feuille plus petite que Rogers H109. Tous ces motifs sont incontestablement attribuables à l'œuvre de Cinnamus (135-170). De plus, le style du Dr. 30 de SteenePitgam se rapproche fortement au style d'un Dr. 30 avec signature intradécorative rétrograde CINNAMI, trouvé à Cooling sur l'île de Graine (PGC, Pl. 174, 1). Style de Cinnamus.

46. (Inv. S3) Dr. 37. Fragment de paroi. Ove peu lisible. La ligne à ondes larges et la rosette pourraient indiquer le style de Tetturo (vers 135-165 après J.-C.). Pour la datation : voir Alcester (p. 94, $\mathrm{n}^{\circ} 12$ ). Peut-être style de Tetturo, vers 135-165.

47. (Inv. S2) Dr. 37. Fragment de paroi. Ove Rogers B 162 : ove à coeur étroit avec orle double, à droite : dard simple : Sissus II. Style de Sissus II (135-165 après J.-C. selon LondonBillingsgate, p. 61, CG86 mais 145-170 selon London-NFW, p. 148, fig. 79).

48. (Inv. S7) Dr. 37. Fragment de paroi. Deux tessons du même individu. Frise d'oves à coeur avec orle double. Dard à droite, striée et à tête globuleuse, rentrant : ove Rogers B50. Astragale Rogers R3. Tous les deux appartenant au répertoire de Cintinus. Style de Cintinus.

49. (Inv. S1-1996) Dr.37. Fragment de paroi. Frise d'oves à coeur filiforme abîmé, avec orle double. Dard mal venu à droite Simpson et Rogers ove 3. Néanmoins, les oves sur notre tesson sont estampillés sur une ligne lisse. Cette habitude se trouve que chez le potier Pugnus, qui a utilisé l'ove en question (PGC, p. 258 et fig., 55 , ove 4 et Dover, 334). Sur la frise : décor en style libre avec lionne à droite, pattes avant d'un autre animal. Pour un décor similaire : PGC : Pl. 155, 27 : Pugnus. Style de Pugnus, vers 140-170.

50. (Inv. S2) Dr. 37. Fragment de paroi. sur la ligne de base : héron plus petit qu'Osw. 2197; PGC: $\mathrm{Pl}$. 154, $16:$ Pugnus; Ovilava : Taf. 56, 4-5: Pugnus. Style de Pugnus, vers 140-170.

51. (Inv. S9) Dr. 37. Fragment de bord. Ove à coeur filiforme, orle double et dard à gauche, terminant dans un trifol : $P G C$ : fig. 39, 4 : Servus III; Pl. 138, 1-6: Servus III. Style de Servus III vers $140-160$ (PGC, p. 280).

52. (Inv. S2) Dr. 37. Fragment de bord. Ove Rogers B17 : ove à coeur et à orle double. Dard à gauche, terminant dans une étoile : Servus III. Style de Servus III vers 140-160.

53. (Inv. S7) Dr. 37.Fragment de paroi. Décor en style libre, scène de chasse (?). Frise d'oves à coeur central et orle double, dard à tête globuleuse, accolé à gauche Rogers B17 : Servus III. Ours à droite: Osw. 1578: PGC: $\mathrm{Pl} .82,5:$ Sacer $: \mathrm{Pl} .92,16$ : Docilis; Pl. 106, 22 : Paternus (avec tortillon); Pl. 118, 13 Criciro. Lionne bondissant à gauche : Osw. 1527; PGC Pl. 86, 15 : Attianus; Pl. 118, 9; 117, 3 : Criciro, Pl. 163, 65,70: Cinnamus; Velzeke V113 : Criciro. Tortillon : PGC fig. 30, 21 : Puternus II. Probablement style de Servus III.

54. (Inv. S2) Dr. 37. Fragment de paroi. Décor de métopes constituées par des cordons perlés. Cupidon à droite (plus grand que Osw. 508), tenant un objet méconnaissable. Cupidon à gauche (plus petit qu' Osw. 504). Bouclier : PGC : Pl. 112,7 et Pl 114,28 et $35:$ Advocisus. Les deux types de Cupidon figurent également sur un bol Dr. 37, trouvé à Wroxeter $(\mathrm{Pl} .33, \mathrm{H} 3)$ et signé ADVOCISI. Style d'Advocisus, vers 160-190.

55. (Inv. S2) Dr. 37. Fragment de paroi. Décor de métopes consti- tuées par des cordons perlés. Mercure : Osw.543. Au-dessus : motif Rogers U103 : e.a., Advocisus, Divixtus. Petite rosette (PGC, fig. 33,3 : Advocisus). Composition comparable à Vendeuil (Pl. 11, 196). Style d'Advocisus.

56. (Inv. S7) Dr. 37. Fragment de paroi. Décor de métopes au moyen de lignes perlées. Dans un médaillon à double bordure unie : tortue $O s w .2156$. Dauphin à droite $O s w$. 2382. Le finissage net, les rangées de perles et la combinaison avec la petite rosette Rogers $\mathrm{C} 122$, plaident en faveur du style d'Advocisus. Style d'Advocisus.

57. (Inv. S7) Dr. 37. Fragment de paroi. Décor de métopes au moyen de lignes perlées. Médaillons à double bordure unie. Petite rosette Rogers C122 : Belsa, Divixtus, Advocisus. Style d'Advocisus.

\section{c) Antonin tardif (ca 170-200)}

1. Groupe de CENSORINUS, 150-180 (CGP, p. 192) ou 160-190 (London-NFW, p. 152, 2.26) PATERNUS II et LAXTUCISSA

58. (Inv. S1) Dr. 37. Fragment de bord. Ove à coeur large, orle plus au moins carénée. Dard à droite, s'élargissant vers l'extrémité comme à Ovilava: Taf. 45, 2, 6: Censorinus; $P G C$ : fig. 29,2 : Censorinus mais aussi Paternus, Albucius, Laxtuccissa et Mammius ; PGC : Pl. : 103, 19-20 : Censorinus. La frise d'oves est bordée d'une ligne d'astragales comme $P G C: \mathrm{Pl} .102,11$, 13-18 et PI. 103, 19-20: Censorinus; Ovilava: Taf. 44, 4, 5 et fig. $45,1-4,6$. L'habitude de border la frise d'oves avec une ligne d'astragales se retrouve fréquemment dans l'oeuvre de Censorinus. Style de Censorinus.

59. (Inv. S1) Dr. 37. Fragment de bord. Ove à coeur avec orle double. Dard à droite, s'élargissant vers l'extrémité (tête de marteau) : ove Rogers B206; commun à Quintilanus. Laxtucissa, Censorinus et Paternus II. Cordon d'astragales. Décor de métopes constituées de lignes ondulées attachées à des rosettes à 7 pétales. Médaillon à double bordure unie. Guerrier ou Mars debout, armé d'un poignard et d'un bouclier : Osw. 152, un type-figuré qui se trouve aussi dans l'oeuvre de Censorinus: Ovilava Taf. 45, 2. Style de Censorinus.

60. (Inv. S1) Dr. 37. Fragment de paroi. Ove à coeur avec orle double. Dard à tête globuleuse et perforé à droite. Amour ou cupidon assis sur un petit socle et tourné à gauche : Osw. 444; PGC : Pl. 101, 7 et Pl. 102, 13 : Censorinus; Ovilava: Taf. 45, 6 : Censorinus. Style de Censorinus.

61. (Inv. S2) Dr. 37. Fragment de paroi. Décor en métopes au moyen de lignes perlées. A gauche : scène érotique $O s w . \mathrm{M}$, comme PGC : Pl. 101, 10; 102, 11 : Censorinus; Pl. 100,25 : Laxtucissa ; Pl. 80, 21 : Acaunissa. Petit cercle lisse : fréquemment employé dans l'officine de Censorinus comme par exemple : $P G C$ : fig. 29,6. Les petits cercles et la scène érotique se trouvent associés dans l'oeuvre de Censorinus comme le montre $P G C: \mathrm{Pl}$. 102, 11. A droite : double cercle lisse avec lièvre Osw. 2116 à gauche. Style de Censorinus.

62. (Inv. S2) Dr. 37. Fragment de paroi. Décor en métopes, constituées de lignes perlées. Oiseau à droite : Osw. 2239D; $P G C$ : Pl. 102,14 : Censorinus. Motif végétal, indéterminć. Probablement style de Censorinus.

63. (Inv. S1) Dr. 37. Fragment de fond. Décor de métopes (?). Aigle Osw. 2183 fréquemment utilisé dans l'oeuvre de Censorinus et Laxtucissa comme PGC: Pl. 102, 11, 18; PI. 103, 19 : Censorinus; Ovilava: Taf. 45, 2, 6 : Censorinus, Taf. 41,1 : Laxtucissa (pattes plus larges que S1-11), Etaples: PI. 11.1 : Censorinus. A gauche et à droite : peut-être un médaillon double comme à Ovilava : Taf. 15,2 : Censorinus; PGC : Pl. 102, 11 : Censorinus; fig. 29,7 : Censorinus, fig. 30,17: Paternus. Style de Censorinus.

64. (Inv. S1) Dr. 37. Fragment de paroi. Décor de métopes et médaillons (?). Dans un grand médaillon : doubles cercles concentriques : $P G C$ : fig. 29,7 : Censorinus: fig. 30,17 : Paternus. Au dessus : pattes de devant d'un animal bondissant (indéterminé). Le cercle est typique pour l'oeuvre des officines de Paternus II et Censorinus. 
65. (Inv. S7) Dr. 37. Fragment de paroi. Ove Rogers B206 : ove à coeur et à orle double, à gauche : dard à tête de marteau $(P G C$. fig. 30, ove 3) : Paternus. La frise comprend un décor à grands rinceaux, comme $P G C: \mathrm{Pl}$. 108. 37 : Paternus. Ce fragment de sigillée est vraiment très frappant à cause de sa qualité exceptionnelle : une surface brillante. un estampillage très net et une argile bien cuite. Style de Paternus II.

66. (Inv. S2) Dr. 37. Fragment de paroi. Décor de métopes. Ove à coeur large avec orle double. Dard à droite, légèrement rentrant. Dans un feston lisse : sphinx à gauche : Osw. 857; Vendeuil, 336 : Cinnamus; Ovilava : Taf. 69,3 (aile différent) : Cinnamus, Knorr, 1905, Taf. VIII, 2 : Paternus II. Style de Paternus II.

67. (Inv. S6) Dr. 37. Fragment de paroi. Décor libre (?). Ove abîmé et peu lisible. Probablement ove à coeur avec orle double. Dard à droite, s'élargissant vers l'extrémité (tête de marteau?) Rogers B 206 (?). Cordon d'astragales. Triton à gauche, avec épée : Osw. 21. Produit du cercle de Paternus, Censorinus et Laxtucissa. La collaboration artistique et la ressemblance artistique de ces 3 potiers ont déjà été fréquemment attestées.

68. (Inv. S7) Dr. 37. Fragment de paroi. Décor de mćtopes au moyen de cordons perlés. Feston à bordure unie. Médaillon à double bordure unie. Cercle : PGC : fig. 30, 27 : Paternus. Signature intra-décorative, rétrograde PATERN [FE]. Produit de Paternus II.

69. (Inv. S7) Dr. 37. Fragment de paroi. Décor de métopes séparées par des cordons perlés. Dans un médaillon à double bordure unie : amour ou cupidon debout, tenant deux flambeaux : $O s w$ 450; Ovilava, Taf. 48, 2 et Velzeke: V145: Paternus. Ce motif est flanqué par de grands cercles. Petit cercle : $P G C:$ fig. 30 , 27 : Paternus. Dans un médaillon à double bordure unie (CGP : Pl. 104, 10 et Ovilava : Taf. 50, 2 : Paternus) : oiseau aux ailes déployées : Osw. 2324. Style de Paternus II.

70. (Inv. S1) Dr. 37. Fragment de paroi. Décor de métopes constituées d'un cordon d'astragales, timbrés d'étoiles. Triton à gauche ou à droite, variant Osw. 19 ou 21 comme à Vendeuil : 233, 246, 282, 316 : e.a. Laxtucissa; PGC : Pl. 99, 18 : Laxtucissa ; Pl. 101,4 : Censorinus; Pl. 112, 2, 10 : Advocisus : Ovilava: Taf. 54,6: Paternus, Taf. 42,1: Laxtucissa. Taf. 49,1 Paternus. Le métope est bordé d'un cordon d'astragales, terminant dans une demie rosette comme à Vendeuil : 283 : Laxtucissa, Doeccus; PGC : Pl. 100, 25 : Laxtucissa; Ovilava : Taf. 44,1: Laxtucissa; Taf. 44,4 : Censorinus. L'usage des cordons d'astragales placés verticalement, figure souvent dans l'œuvre de Laxtucissa (PGC, p. 185). Style de Laxtucissa.

71. (Inv. S1) Dr. 37. Fragment de paroi. Décor en métopes constituées de lignes à perles carénées. Médaillon lisse. Tortillon PGC : fig. 27,4 : Laxtucissa. Colonne Rogers P23 utilisée par la majorité des potiers de Lezoux (plus particulièrement Albucius) ayant travaillé dans la deuxième moitié du deuxième siècle. $P G C$ : fig. 27.13 : Laxtucissa. Peut-être style Laxtucissa.

72. (Inv. S4) Dr. 37. Fragment de paroi. Décor en style libre, assez mal venu et peu lisible. Cheval de mer à gauche : Osw. 33. Petit médaillon cordé Rogers E54 ou 71;CGP : fig. 31,7: Iustus et Pl. 146, 12 : Mercator II; mais aussi : Rogers E71. Rinceau ou médaillon à bordure triple comme à Leicester (fig. 7,7) dans le style de Mercator II, ainsi qu'un motif inconnu, peut-être un triton. Probablement style de Mercator 11 , vers 160-190.

73. (Inv. S1) Dr. 37. Fragment de paroi. Ove à coeur, orle double avec dard strié à droite : $P G C$ : fig. 36, 2 : Iullinus. Décor de métopes séparées par des cordons perlés. Dans un feston à double bordure unie : masque gisant : Osw. 1214 : e.a. Iullinus. Style de Iullinus, vers 160-190.

74. (Inv. S1) Dr. 37. Fragment de paroi. Décor de métopes au moyen de cordons à perles carrées. Grand cercle : $P G C: \mathrm{PI}$. 125, 2 et 8, Pl. 127, 22, 27, 28 : Iullinus. Chapiteau Rogers p. 79 : Iullinus: $P G C$ : fig. 36,6 : Iullinus , avec arcade à double bordure unie : $P G C, \mathrm{Pl} .126 .15$ et 18 et $\mathrm{Pl}, 127: 22$ et 28 Iullinus. Dans un feston à double bordure unie : masque gisant Osw. 1214. Chien courant vers la gauche comme Osw. 1993. Style de Iullinus.

75. (Inv. S2) Dr. 37. Fragment de paroi. Décor en style libre. Scène de chasse : un chasseur avec une lance (Osw. 684A ?) affrontant un animal. Feuille pentaphylle (Ovilava : Taf. 56. 3 Pugnus et Taf. 62. 5 : Severus-Caletus). Probablement style du groupe Severus-Caletus après 160 (PGC, p. 225) ou Banus. vers $170-200$.

76. (Inv. S2) Dr. 37. Fragment de paroi. Décor de métopes séparées par des lignes ondulées. Feston à bordure unie. Cerf galopant à gauche : Osw: 1822N. La ligne aux grandes ondes constitue un des motifs les plus caractéristiques de l'oeuvre du potier Servus II (cf. PGC: Pl. 131 : I et 3-7: Sauvaget 1970, pp. 132133). De plus, le cerf $O s w .1822 \mathrm{~N}$ figure également sur quelques bols de Servus II, trouvés à Lezoux (Sauvaget 1970, Pl. I, 4 et PI. II, 8 et 9). Style de Servus II de Lezoux (160-190 selon London-NFW. p. 155, 2.41: ca 140-175 selon PGC, p. 273.)

77. (Inv. S2) Dr. 37. Fragment de bord. Ove à coeur large, orle double et dard à droite, s'élargissant vers l'extrémité : $P G C, \mathrm{Pl}$. 135, 35, 41 : fig. 40,1 : Casurius. Décor de métopes constituées de lignes perlées. Feuille dentelée comme à Margidunum (Pl. XLII, 2 : Casurius et PGC. Pl. 132, 11 : Casurius. Style de Casurius, vers 165-200 ( $P G C$, p. 238). Antoninus Pius-Clodius A lbinus (Ovilava, p. 206).

78. (Inv. S9) Dr. 37. Fragment de paroi. Frise d'oves assez mal venues et peu lisibles. Ove $P G C$ : fig. 40,1 : Casurius : ove à cocur étroit et double arcature, dard accolé à droite. Décor de métopes au moyen de lignes perlées. A gauche : Minerve à droite, avec bouclier et casque : Osw. 126 : Casurius, comme à Vendeuil : Pl. 11. 204,5; Pl. 12, 218 : Casurius; et à Etaples : Pl. 16,3 faussement attribué au style de Cettus; Kelvedon fig. 47 : Casurius. Dans le panneau droit : strigiles dextrosinistrogyres Rogers U151 : Casurius et Servus III. PGC fig. 40,5: Casurius, et Pl. 138,4 : Servus III: Kelvedon fig. 47 : Casurius. Une pièce similaire (même moule?) a été trouvée à Kelvedon (fig. 47). Les motifs et le décor général sont caractéristiques de l'oeuvre de Casurius.

79. (Inv. S7) Dr. 37. Fragment de paroi. Ove à cœur large, orle double et dard à droite, s'élargissant vers l'extrémité : $P G C, \mathrm{Pl}$. $135,35,41$; fig. 40,1 : Casurius. Dans un feston à double bordure unie : $P G C: \mathrm{Pl} .135,35$ et $36 ; \mathrm{Pl} .136,46 ; \mathrm{Pl} .137: 55$ et 65 : Casurius, cheval marin (plus petit qu'Osw. 33), tourné à gauche. Style de Casurius.

80. (Inv. S2) Dr. 37. Fragment de bord. Ove Rogers B84 : petite ove à cœur filiforme et double bordure unie, à droite : dard à tête globuleuse : Casurius. PGC : fig. 40, ove 3 : Casurius. Style de Casurius.

81. (Inv. S7) Dr. 37. Fragment de paroi. Décor de métopes séparées par des cordons perlés. les motifs sont difficiles à identifier: dans un médaillon à double bordure unie, peut-être la feuille Rogers $\mathrm{H} 47$ de Casurius; en-dessous un poisson (?). Probablement style de Casurius.

82. (Inv. S2) Dr. 37. Fragment de paroi. Décor de métopes séparées par des cordons perlés. Feston à bordure unie. Cercle. Le style est comparable à $P G C: \mathrm{Pl}, 135,36:$ Casurius. Style de Casurius.

83. (Inv. S7) Dr. 37. Fragment de paroi. Décor de métopes au moyen de cordons perlés. Triton brandissant une massue (plus petit qu'Osw. 19). Homme nu, debout, tourné de profil à droite : Osw. 638. Ce type-figuré est tout à fait caractéristique du répertoire du potier Casurius : comme le montrent les exemples dans $P G C$ : Pl. 133 à 137. Style de Casurius.

84. (Inv. S1) Dr. 37. Fragment de paroi. Motifs à relief assez prononcé. Décor en métopes constituées de lignés perlés. A gauche : feuille Rogers H68 ( $P-17$ et Casurius), PGC : fig. 40,10: Casurius et fig. 41,16:Banvus. Motif similaire à Leicester (fig. 8.8) : Casurius et PGC : Pl. 133,14; 136,46,60: 137,60 : style de Casurius. Au milieu et à droite : pilastre composé de perles et d’éléments végétaux comme à Leicester (fig. 8,8): Casurius. Les motifs autant que la disposition générale dı décor sont caractéristiques de l'oeuvre du potier Casurius. Une pièce avec feuille et pilastre similaire trouvé à Leicester, a été attribuée au style précoce de Casurius, c'est-àdire, vers 150 (Leicester. p. 53).

85. (Inv. S3) Dr. 37. Fragment de fond. Motifs à relief prononcé. 
Décor en métopes. Panneaux constitués de lignes perlées, assez bien estampillées et chaque fois terminant dans un petit bol. A droite : Vénus $O s w$. 339. PGC : Pl. 133,19; 134,22 Casurius, Arras : Pl. 13, 74 : Casurius. En dessous et à gauche : petite feuille courbée Rogers H167 employée par Caletus, Casurius, Doeccus et Servus II. Motif identique : $P G C$ : très fréquent aux Pl. 132 à 136 : Casurius. Etaples Pl. 15,5 : Casurius. Sans doute un produit du potier Casurius. Pâte et style de qualité supérieure.

86. (Inv. S2) Dr. 37. Fragment de paroi. Décor de métopes, au moyen de lignes perlées. Panthère à queue fourchue, marchant à gauche. (type-figuré pas compris dans l'index de F. Oswald). Peut-être style de Casurius.

87. (Inv. S1) Dr. 37. Petit fragment de paroi. Décor de métopes. Coquille Rogers U 77. PGC: Pl. 150,39:Doeccus et fig. 44,6: Doeccus. London-Billingsgate : CGXIII, 100 : Doeccus I. Style de Doeccus, vers 165-200 (London-Billingsgate).

88. (Inv. S2) Dr. 37. Fragment de paroi. Décor de métopes au moyen de lignes perlés. Médaillon à double bordure unie. Vase à motif végétal : PGC : Pl. 147, $6:$ Doeccus. Même agencement de décor à London-NFW : 2.49 : Doeccus. Style de Doeccus.

89. (Inv. S2) Dr. 37. Fragment de paroi. Dans un médaillon à double bordure unie Rogers E8 : Doeccus, masque à droite : Osw. 1330. L'usage des médaillons Rogers E8 est un caractère distinct du potier Doeccus, comme le montrent des bols London-NFW : 2.47, South-Shields : fig. 29, 63 et PGC : Pl. 148, 18-19. Style de Doeccus.

90. (Inv. S1-1996) Dr. 37. Fragment de paroi. Motifs à relief prononcé. Décor de métopes, constituées de lignes de perles carrées. Dans un médaillon ou feston à bordure lisse et à bordure perlée : dauphin Osw.2392. Le médaillon ou feston ainsi que le cordon perlé terminant en bol, figure fréquemment dans l'oeuvre du potier Doeccus (London-NFW : 2.47 et 2.48; $P G C$ Pl. 148, 14, 18, 19). Le dauphin se trouve entre autres dans le répertoire décoratif de Doeccus $(P G C: \mathrm{Pl} .147,1)$, également placé dans un feston ou médaillon décrit ci-dessus : SouthShields (fig. 29,63). Cela vaut de même pour le putto $O s w 440$ en-dessous (PGC: Pl. 150, 54 : Doeccus). A gauche : astragale : $C G P$ : fig. 44 : Doeccus. Style de Doeccus.

91. (Inv. S2) Dr. 37. Fragment de paroi. Décor de métopes au moyen de lignes perlées. Ours galopant à gauche : Osw. 127. Au-dessus : motif Rogers U246; PGC : fig. 44,8: Doeccus. Venus ou femme nue, debout : Osw. 281A(?). Style de Doeccus.

92. (Inv. S2) Dr. 37. Fragment de paroi. Poisson à gauche : Osw. 2418. Cheval marin, tourné à gauche : $P G C$ : Pl. 151,57: Doeccus. Style de Doeccus.

93. (Inv. S1) Dr. 37. Fragment de paroi. Motifs à relief prononcé. Décor de métopes constituées de lignes perlées. A gauche : feuille de vigne Rogers H134: Doeccus. PGC: Pl. 150, 42 et 149,33 : Doeccus. A droite : putto assis à gauche : CGP. Pl. 150, 54 : Doeccus; Pl. 102, 12, 14 : Censorinus; Pl. 104, 4, 8; Pl. 105,12 : Paternus; PI. 121, 16 : Albucius; Ovilava : Taf. 45,6 : Censorinus. Ligne unie. Style de Doeccus.

2. Lezoux, deuxième moitié $\mathrm{II}^{\mathrm{e}}$ siècle : produits peu distinctifs de potiers spécifiques

94. (Inv. S2) Dr. 37. Fragment de paroi. Ove Rogers B135 : ove à coeur étroite, orle double et dard à droite, s'élargissant vers l'extrémité : P-17, lustus, Paternus.

95. (Inv. S3) Dr. 30 ou 37. Fragment de paroi. Décor de métopes et feston. Dans un feston lisse : dauphin Osw. 2382 à droite. A droite : aile, peut-être d'une nikè à droite. Cordon perlé, constitué de perles assez fines. A rapprocher du style d'Albucius, Iustus, Doeccus etc.

96. (Inv. S1) Dr. 37. Fragment de paroi. Décor de métopes. Apollon avec lyre, assis sur un klinè : Osw. 83 : Arcanus, Cinnamus; Vendeuil : 196 : Advocisus, 278 : Divixtus, Cinnamus, Cettus. Ce tesson est assez difficile à attribuer au style d'un potier spécifique. La technique indique un produit de haute qualité. Le motif pose des problèmes, surtout en ce qui concerne la jonction entre le dossier et l'accoudoir du kliné. Dans la majorité des pièces publiées, cette jonction est réalisée en forme de croix, c'est-à-dire l'accoudoir et le dossier se prolongent après jointure. Dans le cas du tesson de SteenePitgam, cette jonction est marquée d'un coin arrondi. Malgré le relief pronuncé du motif, cet élément distinctif n'est peut-être que le résultat d'un estampillage insuffisant.

97. (Inv. S1) Dr. 37. Fragment de paroi. Décor de métopes (?). Dans un médaillon double et lisse : oiseau à droite : Osw. 2237A : Mercator.

98. (Inv. S3) Dr. 37. Fragment de paroi. Décor en style libre, scène de chasse (?). Panthère ou chien à droite.

99. (Inv. S2) Dr. 37. Fragment de paroi. Décor en style libre. Taureau de mer à droite : Osw. 52A; PGC, Pl. 145,4 : Mercator I \& II; Pl. 150,41, Doeccus; Pl. 111, 12, 14 : Iustus; Pl. 121,8 : Albucius; Ovilava : Taf. 46,$4 ; 47,6 ; 48,1$ : Paternus. Tortillon double.

100. (Inv. S7) Dr. 37. Fragment de paroi. Minerve : Osw. 126B : Iustus; PGC: Pl. 111, 15: Iustus; Velzeke : V115: Cinnamus. Deuxième moitié $\mathrm{II}^{\mathrm{e}}$ siècle.

101. (Inv. S1) Dr. 37. Fragment de paroi. Ove à coeur avec orle double. Dard à droite, constitué de perles et se terminant dans une rosette : Rogers B32: X-5, X-6. Pugnus, Mapillo. Feston à double bordure unie. Lezoux, milieu et deuxième moitié Il $^{\mathrm{c}}$ siècle.

\section{Les produits de l'est de la Gaule}

\section{a) Les ateliers de l'Argonne}

102. (Inv. S2 vers S3) Dr. 37. Fragment de bord. Ove à coeur filiforme et orle triple. Dard à droite, à tête sortante : VieuxVirton : ove 4, Pl. VI. Renversé sur le tesson de SteenePitgam.

103. (Inv. S2) Dr. 37. Fragment de paroi. Ove à coeur filiforme et orle triple. Dard à droite, à tête sortante : Vieux-Virton : ove 4 , Pl. VI. Fragment de sautoir en lignes tremblées : Vieux-Virton, Pl. XI, D76 ; Butzbach, Pl. 18, 485.

104. (Inv. S2) Dr. 37. Fragment de paroi. Décor en métopes et festons. Surface elliritée. Décor mal venu et peu lisible. Ove à coeur filiforme et orle double, dard à droite à tête globuleuse : Virton : fig. 94bis : ove 16; Chenet \& Gaudron : R3 (fig. 54bis et fig. 57F). La position du dard (gauche ou droite) reste indéterminée. Ligne tremblée, caractéristique de l'Argonne. Feston double avec feuille trifoliée Hofmann 368 : Lavoye. En dessous : partie d'une arcade ou d'un médaillon cordé : motif indéterminé. A gauche : spirale? Pièce de qualité et de style inférieur.

105. (Inv. S2) Dr.37. Fragment de paroi. Ove à coeur filiforme et orle double. Dard à gauche : à tête globuleuse, se rentrant : ove G de Ricken (1934, Taf. XIII, 53-54). Comparez : Oswald (1945, fig. 6, XI) : Germanus. Bacchus avec thyrse : Osw. $518 \mathrm{a}=$ Hofmann, Catalogue, $\mathrm{n}^{\circ} 40:$ Tocca et Tribunus $=$ Vieux-Virton, Pl. VI. H7 et Pl. XV, 61. Lion : Hofmann, Catalogue : A6 et Pl. XV, 61. Pour un style comparable : Velzeke V168. Style de l'ove « $\mathrm{G}$ », Argonne, dernier quart II ${ }^{\mathbb{E}}$ siècle.

106. (Inv. S1) Dr. 37. Fragment de paroi. Ove à coeur avec orle double. Dard à droite, à tête globuleuse, rentrant à gauche : Ricken, « eierstab G» (Taf. XIII, 53); Velzeke V161-171 : Argonne, ove G; Waasmunster : fig. 8,7 : Argonne : deuxième siècle; Vendeuil : 452 (sans description ni détermination de l'ove); Grobbendonk: Afb. 5, 21 : dernier quart deuxième siècle. Style de l'ove «G $\mathrm{G}$, Argonne, dernier quart II $^{\mathrm{c}}$ siècle.

107. (Inv. S2) Dr. 37. Fragment de paroi. Ove à coeur filiforme et orle double. Dard à gauche : à tête globuleuse, rentrant : ove $G$ de Ricken (1934. Taf. XIII, 53-54). Comparez : Oswald 1945, fig. 6, XI : Germanus. Feuille cordiforme avec longue tige : Oswald 1945, fig. 6, XIX : Germanus: Hofmann, Catalogue, $\mathrm{n}^{\circ}$ 376-376A : Germanus; Vieux-Virton, Pl. IX, P7. Le joueur de flûte à gauche ne figure dans aucun répertoire publié. Style de l'ove " G », Argonne, dernier quart II' siècle.

108. (Inv. S1) Dr. 37. Fragment de paroi. Décor de métopes au moyen de lignes perlées. A gauche : médaillon à double 
bordure unie : Virton D 16 (plus petit que S1-25); Hofmann 471 : employé par Tribunus et un élève; Ricken 1934, PI 12,27,62 : style à l'ove C: Arentsburg, fig. 79,4: Argonne Butzbach: Pl. 16, 434 et Pl. 17, 436; Novaesium II, Pl. 4,182 et Pl. 5, 188. Le motif dans le médaillon nous est inconnu. A droite : lion bondissant à gauche : Virton A6 : Argonne; Osw. 1442; Hofmann 169A (tous les potiers ayant succédé à Tocca et Tribunus); Ricken 1934, Pl. 13,26 (oves A et B); Chenet : fig. $62 \mathrm{~A}$ (moule contribué à Tocca). 62B et $63 \mathrm{C}$ (signé Cintugnatus); Fölzer : Pl. 28.369 : Lavoye; Arentsburg : fig. 79, 4, 39, 45; Argonne: Vieux-Virton : fig. 44, 124 : Argonne, deuxième moitié deuxième siècle : tous les potiers ayant succédé à Tribunus et Tocca. Entre le médaillon et le panneau au lion : feuille lancéolée Hofmann 376A Germanus. Lavoye, cercle de Germanus, Tocca, Tribunus : milieu et troisième quart du $\mathrm{II}^{\mathrm{c}}$ siècle.

109. (Inv. S2) Dr. 37. Fragment de paroi. Ove à coeur filiforme et orle double. Dard à gauche : à tête globuleuse, rentrant : ove $\mathrm{G}$ de Ricken (1934, Taf. XIII, 53-54). Comparez : Oswald 1945, fig. 6, XI : Germanus. Décor de métopes séparées par des corduns perlés el des rusaces. Mulif nabellifurme. Molif de pelta? Style de l'ove «G », Argonne, dernier quart $\mathrm{II}^{\mathrm{e}}$ siècle.

110. (Inv. S1) Dr. 37. Fragment de paroi, Surface assez effritée. Décor de métopes constituées de lignes perlées (carrées), timbrées de demi-cercles cordés. Perles carrées et demicercle cordé comme à Virton : D13 : e.a. Eburus : fig. 72,73,74,86,109; Hofmann 477-478: Eburus; Chenet et Gaudron: fig. 18;19,10,13,14,21-26;25,51: « le gobeletier » (=Eburus). A gauche et à droite : motif assez mal venu et presque illisible, peut-être similaire à la scène érotique Hofmann 135 (diminuée) : Tocca; Vieux-Virton : H29 : Tocca; St. Mard : fig. 44,122. Le type-figuré sur le tesson de Steene-Pitgam, est probablement un surmoulage du motif original de Tocca. Style d'Eburus (?) de Lavoye, vers 150-175.

111. (Inv. S1) Feuille nervurée : Hofmann 371: Gesatus ou Germanus de Lavoye.

112. (Inv. S2) Dr. 37. Bas de panse. Décor de lignes tremblées : Chenet \& Gaudron : fig. 62.4; Oswald, 1945 : fig. 8,28. Deuxième moitié II ${ }^{\mathrm{e}}$ siècle.

113. (Inv. S1) Dr. 37. Bas de panse. Décor de lignes tremblées, terminant dans des bols : e.a. Chenet \& Gaudron : fig. 62,4; Oswald, 1945 : fig. 8,28. Deuxième moitié II $^{\mathrm{e}}$ siècle.

114. (Inv. S8) Dr. 37. Bas de panse. Frise inférieure de rosettes et spirales; pour un décor avec arrangement semblable Asciburgium 2, Taf. 10,61 et Vendeuil-Caply II, Pl. 19, 394.

115. (Inv. S2) Dr. 37. Bas de panse. Engobe originale disparue Médaillon à bordure unie. Au milieu : oiseau : Ricken 1934 , Taf. XII, 53 et Taf. XVI, 13; Hofmann, Catalogue, $\mathrm{n}^{\circ} 328$; Vieux-Virton : PI. IX : A26. Rosette : Ricken 1934, Taf. XIII, $3(?)$.

116. (Inv. S3) Dr. 37. Fragment de paroi. Décor bordé d'une frise de rosettes comme Virton D65:38,91,117,125; Hofmann 46; Ricken 1934, Pl. 13,1 (avec oves A et B), Pl. 12, 2 (avec ove C); Butzbach : Pl. 17, 436, 441, 463 : Argonne; Vendeuil : 424, 460 : Argonne; Arras : Pl. 14, 88-81 : Argonne. Argonne, milieu et deuxième moitié II $^{\mathrm{c}}$ siècle.

117. (Inv. S1) Dr. 37. Fragment de paroi. Frise inférieure de rosettes : Vendeuil-Caply: Pl. 20, 445 et 454, Pl. 22, 490

118. (Inv. S2) Dr.37. Fragment de paroi. Arbre ou feuille : Fölzer 390; Ricken 1934, Pl. XIII, 16; Hofmann 371 : Gesatus, Germanus (?); Vieux-Virton: P9. Les autres éléments décoratifs restent difficiles à identifier à cause de la détérioration de la surface.

\section{b) Rheinzabern}

119. (Inv. S3) Dr. 37. Fragment de paroi. Dans un décor d'arcades : Amor Osw. 455. Ri-Fi : M144 ou M144a, utilisé par des potiers comme Januarius I. Cobnertus, Cerialis (I, III, V), B.F. Attoni, Julius II-Julianus, Belsus I, etc. Les autres motifs restent difficiles à identifier. La technique nous semble plutôt caractéristique pour la période Antonin-début III $^{\mathrm{e}}$ siècle (cf. Bird, 1993, p. 2).
120. (Inv. S1) Dr. 37. Fragment de paroi. A gauche dans un double médaillon lisse : âne Osw. 1759. Ri-Fi. : T82: Cobnertus III, Cerialis VI, Comitialis V (très fréquent dans l'oeuvre de ce dernier potier comme par exemple : $L u-R i$ : Taf. 96-102; BadWimpfen : 308 : Comitialis IV ou V. Médaillon RiFi K19 ou $\mathrm{K} 19 \mathrm{a}$ : utilisé par la majorité des potiers de Rheinzabern. A droite : motif carré ; peut-être une estampille intra-décorative. (Lud-Rick. : Taf. 96,6 : Latinni, associé à Comitialis V) et Vénus à gauche : RiFi : M40 : Comitialis V, Julius II-Julianus I: Osw. 284 : Iulius, Comitialis. A l'extrême droite : panthère à droite : Osw. 1518; Ri-Fi : T35 : e.a. Comitialis $V$; London-NFW : 2.97 : Comitialis V-VI. Style de Comitialis V ou de Latinus de Rheinzabern, fin deuxième, début $\mathrm{III}^{\mathrm{e}}$ siècle.

121. (Inv. S7) Dr. 37. Fragment de paroi. Médaillon à double bordure unie, avec masque : Osw. $1336=R i-F i:$ M27; Ovilava : Taf. 180,5 : Victor I. Victor I de Rheinzabern, première moitié III siècle.

122. (Inv. S1) Dr. 37. Fragment de paroi. Décor de métopes constituées de lignes perlées (perles carrées). Cordons perlés RiFi O2 : Julius II-Julianus I, Victorinus II, E48-49, Respectinus I et II, Marcellinus; London-NFW : 2.117 (identique) : Julius II-Julianus I. Julius II-Julianus $I$ de Rheinzabern, après 225 (Simon, 1968, p. 22 et Bernhard, 1981 Abb 3.).

\section{c) Trèves}

123. (Inv. S1-1996) Dr. 37. Fragment de bord. Frise d'oves à coeur large et orle double. Cette ove est utilisée par Afer, Dubitatus et Paternianus de Trèves. (London-NFW: 2.84). A droite : chien courant à droite : Oswald $1962=$ Fölzer 649 $=$ Butzbach: $972,974,975,999,1000$. A gauche : lièvre courant à droite : Fölzer, Taf. 17, 4; Butzbach : 971, 972, 974, 1000. Le chien et le lièvre sont souvent attestés ensemble sur des bols dans le style du potier Dubitatus comme le montre l'exemple de Langenhain (137 et 140) : Dubitatus. Dubitatus de Trèves, vers 225-250. (Bird, 1993, p. 3).

124. (Inv. S1) Dr. 37. Fragment de paroi. Médaillon dentelé : Fölzer 825 : Trèves; London-NFW : 2.73 : Afer-Marinusgroupe de Trèves; Velzeke V183-9: Trèves : période Caracalla-Gordien III. Puttus à gauche : Trèves Werkstatt I : M24; Fölzer S19 : Trèves (Taf. XV, 38; XVII, 16; XVIII, 14); London-NFW : 2.73 : Afer-Marinus groupe de Trèves; Waasmunster fig. 7,6 : Trèves, fin deuxième, début troisième siècle; Lutz P73 : Satto-Saturninus. La technique se rapproche fortement de celle des bols de la même époque, mais produits à Lezoux. Seulement les motifs et l'absence de mica dans l'argile de Trèves sont des éléments suffisamment déterminants pour faire la distinction entre les produits des deux centres dans cette époque. Cette similitude technique a déjà été remarquée ailleurs (Bird, 1993, p. 2). Sans doute un produit du groupe Afer-Marinus de Trèves, vers 200-225.

\section{d) Tessons provenant des ateliers de l'Est mais dif- ficiles à attribuer à un atelier spécifique}

125. (Inv. S1) Dr. 37. Fragment de paroi. Rangée d'acanthes au lieu d'oves : Ricken 1934, Taf. XIV, 7 : potier inconnu. Se rapproche aux ateliers de l'Argonne et du « Virtus-ware » de La Madeleine. Motif pareil à Mogontiacum (Mainz) (Taf. 17,5) : potier inconnu.

126. (Inv. S7) Dr. 37. Fragment de paroi. Engobe disparue. Ove à cœur, orle double (ou triple), dard à tête globuleuse accolé à gauche. Arcade ou médaillon à bordure dentelée. Argonne?

127. (Inv. S2) Dr. 37. Bas de panse. Décoration au moyen de lignes grossières cordées. Trifol à feuilles extérieures striées : Ricken, 1934, Taf. VIII, 14. Argonne?

128. (Inv. S2) Dr. 37. Fragment de paroi. Médaillon dentelé avec buste : Novaesium II : Taf. 13, 281; Grobbendonk: Pl. XVI, 185. Comparable aux produits de Trèves (Zwammerdam, Taf.68, nr.617), mais techniquement ce tesson semble mieux s'associer à la production de l'Argonne. Argonne, deuxième moitié II siècle? 


\section{Bibliographie}

Alcester $=$ Hartley, Pengelly \& Dickinson, 1994.

Arentsburg = Holwerda. 1923

Arras $=$ Bellanger $\&$ Piton, 1981 .

Asciburgium = Vanderhoeven, 1974.

Bad-Wimpfen = Hartmann, 1981 .

Bailleul = De Ciercy \& Deschieter, 1996a

Braughing = Bird et Dickinson, 1988.

Butzbach = Müller, 1968.

Burladingen-Hausen = Knorr, 1919.

Caerleon = Boon, 1986.

CGP $=$ Stanfield \& Simpson, 1958.

Corbridge $=$ Dore, Dickinson, Hartley \& Hartley, 1988.

Dover $=$ Bird \& Marsh, 1981

Etaples $=$ Piton, 1985 .

Exeter $=$ Dannell, 1991.

Grobbendonk = Vanderhoeven. 1977.

Iuvavum = Karnitsch, 1971 .

Kelvedon $=$ Rodwell, 1988.

Langenhain = Simon \& Köhler, 1992.

London-Billingsgate = de la Bédoyère, 1986.

London-NFW = Bird, 1986.

Leicester $=$ Oswald, $1948 \mathrm{a}$.

Lu-Ri = Ludowici \& Ricken, 1948.

Margidunum $=$ Oswald, $1948 \mathrm{~b}$.

Medway $=$ Detsicas, 1968.

Mogontiacum = Baatz, 1962.

Novaesium II = Schönberger \& Simon. 1966.

Ovilava $=$ Karnitsch, 1959

Pompei $=$ Atkinson, 1914 .

PGC $=$ Stanfield \& Simpson, 1990 (réed.).

Ri-Fi = Ricken \& Fischer, 1963.

Saalburg = Ricken, 1934

Sorviodurum $=$ Walke, 1965

South-Shields = Dore, Greene \& Johns, 1979.

Tongeren = Vanvinkenroye. 1989 .

Trèves Werkstatt I = Huld-Zetsche, 1972

Usk $=$ Johns, 1993

Utrecht $=$ Brunsting \& Kalee, 1989

Valkenburg $=$ De Groot, 1960 .

Velzeke $=$ Rogge, 1978.

Vendeuil $=$ Piton, 1990 .

Verulamium I = Hartley, 1972.

Verulamium III = Dickinson, 1984 .

Vervoz $=$ Willems, $1981-1982$.

Vieux-Virton = Raepsaet-Charlier \& Clausse, 1977-1978.

Waasmunster $=$ Thoen, 1967.

Wroxeter $=$ Atkinson, 1942 .

Zwammerdam $=$ Haalebos, 1977.

Atkinson D., 1914. «A hoard of Samian Ware from Pompeii ». Journal of Roman Studies, 4, p. 26-64.

Atkinson D., 1942. Report on the excarations at Wroxeter (The Roman city of Viroconium) in the county of Salop, 19231927. Birmingham.

Baatz D., 1962. Mogontiacum. Neue Untersuchungen am Römische' Legionslager in Mainz. Limesforschungen, Band 4. Bayard D., 1980. "La commercialisation de la céramique commune à Amiens du milieu du Ir à la fin du III $^{\circ}$ siècle après J.-C. ", Cahiers Archéologiques de Picardie, 1, p. 147-209.
Bélot E. \& Canut V., 1994. Céramiques des $2^{c}$ et $3^{e}$ siècles de notre ère issues des fouilles du "Terrain Landrot » à Boulognesur-Mer. Premier aperçu provisoire des données céramologiques boulonnaises concernant le Haut-Empire à la lumière des fouilles rérentes. Fléments d'Archéologie Boulonnaise, 2. Bellanger G. \& Piton D. 1981. "La céramique sigillée des fouilles d'Arras (Baudimont) », Bulletin de la Commission Départementale des Monuments Historiques du Pas-de-Calais, IX, 1, p. 1-39.

Bernhard H., 1981. "Zur Diskussion um die Chronologie Rheinzaberner Relieftöpfer ». Germania, 59, p. 79-93.

Bet Ph. \& Montineri D., 1990. «La sigillée lisse de Lezoux, typologie des formes du Haut-Empire », Revue Archéologique Sites, 43, p. 3-10.

Bet Ph. \& Brulet M., 1994. «La terre sigillée», dans : BRULET R. (éd.). Braives-la-Romaine. Bilan de vingt ans de recherches archéologiques dans l'agglomération gallo-romaine de Braives.1973-1992. Collection d'Archéologie Joseph Mertens, 9, Louvain-La-Neuve, p. 103-109.

Bird J. \& Marsh G., 1981. "The Samian Ware », dans : PHILP B., The Excavation of the Roman Forts of the Classis Brittanica at Dover, 1970-1977. Kent Monograph Series, 3th Report, p. 178-202.

Bird J. \& Dickinson B., 1988. «The Samian Ware », dans : PotTer T. W. \& Trow S. D., Puckeridge-Braughing, Herts. : the Ermine Street excavations, 1971-1972. Hertfordshire Archaeology, 10, p. 97-109.

Bird J., 1986. "Samian Ware », dans : Miller L., RHODES M. \& SChofield J. The Roman Quay at St. Magnus House London. London \& Middlesex Archaeological Society, Special Paper, 8, p. 139-185.

Bird J., 1993. « IIIrd-Century Samian in Britain », Journal of Roman Pottery Studies, 6, p. 1-14.

Boon G. C., 1986. « The Samian Ware », dans : ZIENkIEwICZ J. D., The Legionary Fortress Baths at Caerleon. II. The Finds, p. $39-47$

Brunsting H. \& Kalee A., 1989. « Terra Sigillata met reliëfversiering », dans : Ozinga L. R. P., Hoekstra T J., DE WEERD M.D. \& WYNIA S.L. (eds). Het Romeinse Castellum te Utrecht, p. 121-138.

Cabal M., 1962-1967. " Trouvailles archéologiques aux Noires-Terres à Ardres ", Bulletin de la Société Académique des Antiquaires de la Morinie, 20, p. 638-646.

Cabal M. \& Retkowski-Biville N., 1987. " Marques de potiers sur céramique sigillée trouvées à Ardres », Revue du Nord, LXIX, 272, p. 17-43.

Chenet C. \& Gaudron G., 1955. La céramique sigillée d'Argonne des II' et IIr' siècles. Supplément à Gallia, 6, Paris.

Cools E., 1985. « De Romeinse kustverdediging van maritiem Vlaanderen. Werkhypothesen voor een systematische prospectie ", Westvlaamse Archaeologica, 1, p. 16-27.

Creus I., 1975. De Gallo-Romeinse nederzetting onder het laat-Romeins grafveld van Oudenburg. Archaeologia Belgica, 179.

Dannell G. B., 1971. « The Samian Pottery », dans : CunlifFE B. Excavations at Fishbourne, 1961-1969. Vol. II. The Finds. Reports of the Research Committee of the Society of Antiquaries of London, 27, p. 260-315.

Dannell G. B., 1991. « Decorated Samian », dans : HOLBROOK B. \& Bidwell P.T., 1991. Roman Finds from Exeter. Exeter Archaeological Reports, Vol. 4, p. 55-71.

De Clercq W. \& Deschieter J., 1996a. « La céramique sigillée provenant de la prospection d'une villa gallo-romaine à Bailleul (.Nord) ", Cahier d'Histoire Locale. Section Bailleuloise d'Histoire Locale, 6, p. 27-31.

De Clercq W. \& Deschieter J., 1996b. Terre sigillée prove- 
nant de la prospection d'une «villa» gallo-romaine à Cassel (France, Dép. du Nord). Rapport non-publié.

De Groot J., 1960. "Masclus von La Graufesenque ", Germania, 38, p. 55-65.

De la Bédoyère G. 1986. The Roman site at Billingsgate Lorry Park, London. A Catalogue of Samian and other Finds. BAR BS, 154.

Delmaire R., 1976. Etude archéologique de la partie orientale de la Cité des Morins (civitas Morinorum). Mémoires de la Commission Départementale des Monuments Historiques du Pas-de-Calais, 16. Arras.

Déchelette F., 1904. Les vases céramiques ornés de la Gaule Romaine. Tome 2. Paris.

Detsicas A. P., 1968. "Samian Ware from the Medway Marshes », Archaeologia Cantiana, 82, p. 235-245.

Dickinson B., 1984. "The Samian Ware ", dans : Frere S., Verulamium Excavations, Vol. III. Oxford University Committee for Archaeology, Monographs, 1, p. 175-199. Oxford.

Dore J., Greene K. \& Johns C., 1979. «The Decorated Ware », dans : DORE J. \& GILlaM J. The Roman Fort at South Shields. Excavations 1875-1975, Newcastle, p. 107-127.

Dore J. N., Dickinson B., Hartley B. R. \& Hartley K. F., 1988. "The Pottery», dans : BISHOP M. C. \& DoRE J. N., Corbridge. Excavations of the Roman Fort and Town, 19471980. London, p. 219-286.

Fölzer E., 1913. Die Bilderschüsseln der Ostgallischen Sigillata-Manufakturen. Bonn.

Galliou P \& Sanquer R., 1979. " La sigillée décorée de Kerilien en Plouneventer (Finistère) 》. ${ }^{\text {er }}$ supplément à Archéologie en Bretagne, Brest.

Going C. J., 1992. « Economic « long-waves » in the Roman period? A reconnaissance of the Romano-British ceramic evidence ", Oxford Journal of Archaeology, 11, 1, p. 93-117.

Greene K. T., 1975. " The Samian Pottery », dans : CASEY J., Excavations outside the North-East gate of Segontium, 1971. Archaeologia Cambrensis, 123, p. 54-77.

Haalebos J. K., 1977. « Zwammerdam-Nigrum Pullum. Ein Auxiliarkastell am Niedergermanischen Limes ", Cingula, 3.

Haalebos J. K., 1993. « La Graufesenque et les Pays Bas », Les Annales de Pégasus 1990-1991. Recherches Européennes sur la Graufesenque, p. 26-33.

Hartley B. R., 1972. « The Roman occupation of Scotland : the evidence of the Samian ware », Britannia, 3, p. 1-55.

Hartley B. R., 1972. "The Samian Ware », dans : FrERE S., Verulamium Excavations, Vol. I. Reports of the Research Committee of the Society of Antiquaries of London, 28, p. 216-262.

Hartley B. R., 1985. "The Samian Ware ", dans : PITTs L. F. \& ST. JOSEPH J. K. Inchtuthil. The Roman Legionary Fortress. Britannia Monographs, 6, p. 314-322.

Hartley B. R., 1993. "The new Index of Potters Stamps on Samian Ware (terra sigillata) », Les Annales de Pégasus 19901991. Recherches Européennes sur la Graufesenque, p. 18-25. Hartley B. R., Pengelly H. \& Dickinson B., 1994. "Samian Ware », dans : Cracknell S. \& MAHANY Chr. Roman Alcester: Southern Extramural Area. 1964-1966 Excavations. Part 2. Finds and Discussion, p. 93-119.

Hartmann H. H., 1981. "Die Reliefsigillata aus dem Vicus Wimpfen im Tal », dans : CZYSZ W., KaISER H., Mackensen M., Ulbert G., Hartmann H. H., Römische Keramik aus Bad-Wimpfen. Forschungen und Berichte zur Vorund-Frühgeschichte in Baden-Wurtemberg, 11.

Hermet F., 1934. La Graufesenque (Condatomago). Paris.

Hofmann B., 1968. "Catalogue des poinçons pour moules à vases des décorateurs argonnais ». Ogam, XX.
Hofmann B., 1988. L'atelier de Banassac. Revue Archéologique Sites, Hors-Série 33.

Holwerda, J. H., 1923. Arentsburg. Een Romeinsch militair vlootstation bij Voorburg. Leiden.

Hosdez Ch. \& Jacques A., 1989. « La nécropole à incinérations de Baralle (Pas-de-Calais) ». Nord-Ouest Archéologie, 2. Huld-Zetsche I., 1972. Trierer Reliefsigillata. Werkstatt $I$. Frankfurt-am-Main.

Hurtrelle J. \& Jacques A., 1983. « Arras : fouilles de sauvetage avant la construction de l'Hôtel du Département "., Bulletin de la Commission Départementale d'Histoire et d'Archéologie du Pas-de-Calais, XI, 3, p. 259-336.

Hollevoet Y., 1989. Archeologisch noodonderzoek in de Zeebrugse achterhaven : de Romeinse vondsten. Westvlaamse Archaeologica, 5, p. 33-48.

Johns C. M., 1993. "The Decorated Samian », dans : MANNING W. H. (ed.). Report on the Excavations at Usk 1965 1976 : The Roman Pottery, p. 161-203. Cardiff.

Karnitsch P., 1955. Die verzierte sigillata von Lauriacum (Lorch-Enns). Forschungen in Lauriacum, 3.

Karnitsch P., 1959. Die Reliefsigillata von Ovilava. Linz.

Karnitsch P., 1971. Sigillata von Iuvavum. Die reliefverzierte Sigillata im Salzburger Museum Carolino Augusteum. Salzburg.

Knorr R., 1905. Die verzierten Terra-Sigillata-Gefässe von Cannstatt-Köngen. Stuttgart.

Knorr R., 1907. Die verzierten Terra-Sigillata-Gefässe von Rottweil. Stuttgart.

Knorr R., 1912. Südgallische Terra-Sigillata-Gefässe von Rottweil. Stuttgart.

Knorr R., 1919. Töpfer und Fabriken verzierter Terra Sigillata des ersten Jahrhunderts. Stuttgart.

Knorr R., 1952. Terra-Sigillata-Gefässe des ersten Jahrhunderts mit Töpfernamen. Stuttgart.

Loridant F., 1994. "La céramique commune dans la région de Bavay au Haut-Empire : bilan des connaissances ", dans : TUFFREAU-LIBRE M. \& JACQUES A., 1994. La céramique du haut-Empire en Gaule Belgique et dans les régions voisines : faciès régionaux et courants commerciaux. Actes de la table ronde d'Arras (12 au 14 octobre 1993). Centre de Céramologie Gallo-Romaine. Nord-Ouest Archéologie, 6, p. 29-42.

Ludowici W. \& Ricken H., 1948. Katalog VI. Die Bilderschüsseln der römischen Töpfer von Rheinzabern (Tafelband). Speyer.

Marsh G., 1981. "London's Samian supply and its relationship to the Gallic Samian industry », dans : ANDERSON A. \& ANDERSON S. Roman Pottery Research in Britain and northwest Europe. BAR IS, 123, p. 173-238.

Mees A., 1990. "Verzierte Terra Sigillata aus den Ausgrabungen bei Vechten in den Jahren 1920-1927", Oudheidkundige Mededelingen van het Rijksmuseum voor Oudheidkunde te Leiden, 70, p. 109-181.

Mees A., 1995. Modelsignierte Dekorationen auf Südgallischer Terra Sigillata. Forschungen und Berichte zur Vor- und Frühgeschichte in Baden-Würtemberg, 54. Stuttgart. Morren G. G. A., 1957-1958. « Terra sigillata van de Romeinse nederzetting bij Zwammerdam ", Berichten van de Rijksdienst voor Oudheidkundig Bodemonderzoek, p. 54-76.

Müller G., 1968. Das Lagerdorff des Kastells Butzbach. Die Reliefverzierte Terra Sigillata. Limesforschungen, Bd. 5. Berlin.

Notte L., 1990. «La sigillée d'Harnes (Pas-de-Calais) ». NordOuest Archéologie, 3, p. 7-31.

Oswald F., 1936-1937. Index of figure types on terra sigillata. University of Liverpool, Annals of Archaeology and Anthropology, supplément. Liverpool. 
Oswald F., 1945. « Decorated Ware from Lavoye », Journal of Roman Studies, 35, p. 49-57.

Oswald F., 1948a. "Samian Pottery ", dans : KenYon K. M. Excavations at the Jewry Wall Site, Leicester. Reports of the Research Committee of the Society of Antiquaries of London, 15, p. 43-72.

Oswald F., 1948b. The Terra Sigillata (Samian Ware) of Margidunum. Nottingham.

Piton D., 1985. « La terre sigilléc décorćc à Etaples ", Les Cahiers de Quentovic, 10.

Piton D., 1990. "La Sigillée de Vendeuil-Caply », NordOuest-Archéologie, 3, p. 31-85.

Raepsaet G. \& M.-Th. \& Clausse R., 1977-1978. "Terre sigillée décorée découverte à Vieux-Virton (St. Mard) », Le Pays Gaumais, 38-39, p. 25-106.

Raepsaet G., 1987. « Aspects de l'organisation du commerce de la céramique sigillée dans le Nord de la Gaule au II $^{\mathrm{e}}$ et III $^{\mathrm{e}}$ siècles de notre ère. I. Les données matérielles ", Münstersche Beiträge zur Antiken Handelsgeschichte, Bd. VI, 2, p. 1-79.

Raepsaet G. \& Raepsaet-Charlier M.-Th., 1988. « Aspects de l'organisation du commerce de la céramique sigillée dans le Nord de la Gaule au II et III ${ }^{\mathfrak{e}}$ siècles de notre ère. II. Négociants et transporteurs. La géographie des activités commerciales ", Münstersche Beiträge zur Antiken Handelsgeschichte, Bd. VII, 2, p. 45-69.

Ricken H., 1934. « Die Bilderschüsseln der Kastelle Saalburg und Zugmantel », Saalburg Jahrbuch, 8, p. 130-182.

Ricken H. \& Fischer C., 1963. Die Bilderschüsseln der römischen Töpfer von Rheinzabern (Textband). Bonn.

Rodwell K.A. 1988. The Pre-Historic and Roman settlement at Kelvedon, Essex. Council for Britisch Archaeology, Research Reports, 63.

Rogers G., 1969-1970. « Banassac and Cinnamus », Rei Cretariae Romanae Fautorum, Acta XI-XII, p. 98-106.

Rogers G., 1972. « Un groupe de ratés de four de la Gaule centrale ", Revue Archéologique du Centre, XI, p. 322-326.

Rogers G., 1974. Poteries sigillées de la Gaule Centrale. I. Les motifs non-figurés. Supplément à Gallia, 28. Paris.

Rogge M., 1978. "Kataloog van de vondsten uit de GalloRomeinse nderzettingen van Zottegem-Velzeke », dans : DE Laet S. J., Nenquin J. \& VAN Doorselaer A. Oudheidkundige Opgravingen en Vondsten in Oost-Vlaanderen (O.O.V.O.), VIII, p. 73-186.

Roumegoux Y. \& Termote J., 1993. « De Romeinse periode », dans : Roumegoux Y. \& Termote J. (Red.), KemmelCassel. De vroegste bewoningsgeschiedenis van de Vlaamse Heuvels », Westvlaamse Archaeologica, 9, 2, p. 61-80.

Sauvaget R., 1970. « Le potier Servus II de Lezoux », Revue Archéologique du Centre, 33, p. 127-152.

Schönberger H. \& Simon H.-G., 1966. Novaesium II. Die Mittelkaiserzeitliche Terra Sigillata von Neuss. Limesforschungen, Bd.7, Berlin.

Simon H.-G., 1968. « Das Kleinkastell Degerfeld in Butzbach, Kr. Friedbach (Hessen). Datierung und Funde », Saalburg Jahrbuch, 25, p. 5-64.

Simon H.-G. \& Köhler H.-J., 1992. Ein Geschirrdepot des 3. Jahrhunderts. Grabungen im Lagerdorf des Kastells
Langenhain. Materialien zur römisch-germanischen Keramik, 11. Bonn.

Simpson G. \& Rogers G., 1969. « Cinnamus de Lezoux et quelques potiers contemporains ", Gallia, 27, p. 3-14.

Stanfield J. \& Simpson G., 1958. Central Gaulish Potters. Oxford.

Stanfield J. \& Simpson G., 1990. « Les Potiers de la Gaule Centrale », Revue Archéologique Sites, Hors-Série, $\mathrm{n}^{\circ} 37$. Gonfaron.

Terrisse J.-R., 1968. Les céramiques sigillées gallo-romaines des Martres-de-Veyre (Puy-de-Dôme). Suppl. à Gallia, 28.

Thoen H., 1967. "De Gallo-Romeinse nederzetting van Waasmunster-Pontrave ", Oudheidkundige Repertoria, reeks B., N.C.O.N.B., Brussel.

Thoen H. \& Seillier C., 1970. « La terre sigillée du chantier de Bon Secours à Arras », Septentrion, 1, p. 123-134.

Thoen H., 1978. De Belgische Kustvlakte in de Romeinse tijd. Verhandelingen van de Koninklijke Academie voor Wetenschappen, Letteren en Schonen Kunsten van België. Klasse der Letteren, XL.

Thoen H. \& De Swaef W., 1995. " A terra sigillata cantharos from Erembodegem (Prov. East Flanders) ", Acta Archaeologica Lovaniensia, Monographiae, 8, p. 165-173.

Trimpe-Burger J.A., 1975. "The Islands of Zeeland and South Holland in Roman Times ", Berichten van de Rijksdienst voor Oudheidkundig Bodemonderzoek, 23, p. 135-148.

Tuffreau-Libre M. \& Jacques A.,1985. « La céramique galloromaine du premier siècle dans le sud de l'Atrébatie », Gallia, 43, p. 127-145.

Tuffreau-Libre M. \& Jacques A. (éd.), 1994. La céramique du haut-Empire en Gaule Belgique et dans les régions voisines : faciès régionaux et courants commerciaux. Actes de la table ronde d'Arras (12 au 14 octobre 1993). Centre de Céramologie Gallo-Romaine. Nord-Ouest Archéologie, 6.

Van Beek B. L., 1983. « Salinatores and sigillata : the coastal areas of North Holland and Flanders and their economic differences in the 1st century AD », Helinium, XXIII, p. 3-12.

Vanderhoeven M., 1974. Terra Sigillata aus Mittel- und Ostgallien : Die reliefverzierte Gefässe. Funde aus Asciburgium, Heft 2.

Vanderhoeven M., 1977. De terra sigillata van Grobbendonk. Opgravingen 1971-3. Archaeologia Belgica, 199. Brussel.

Vanvinkenroye W., 1989. Terra Sigillata uit een Romeinse stortplaats te Tongeren. Publicaties van het provinciaal galloromeins Museum te Tongeren, 43.

Vernhet A., 1976. "Création flavienne de six services de vaisselle à la Graufesenque », Figlina, 1, p. 13-27.

Voet M., 1983. «La céramique sigillée à Watten », Bulletin de la société des amis du Vieux Watten et de sa région, 9.

Wacher S. J., 1969. " Excavations at Brough-on-Humber. 1958-1961 », Reports of the Research Committee of the Society of Antiquaries of London, 25, Leeds.

Walke N., 1965. Das römische Donaukastell StraubingSorviodunum. Limesforschungen, Bd. 3, Berlin. Willems J., 1981-1982. "La terre sigillée du vicus belgoromain de Vervoz à Clavier ", Bulletin $d u$ Cercle Archéologique Hesbaye-Condroz, p. 27-82. 


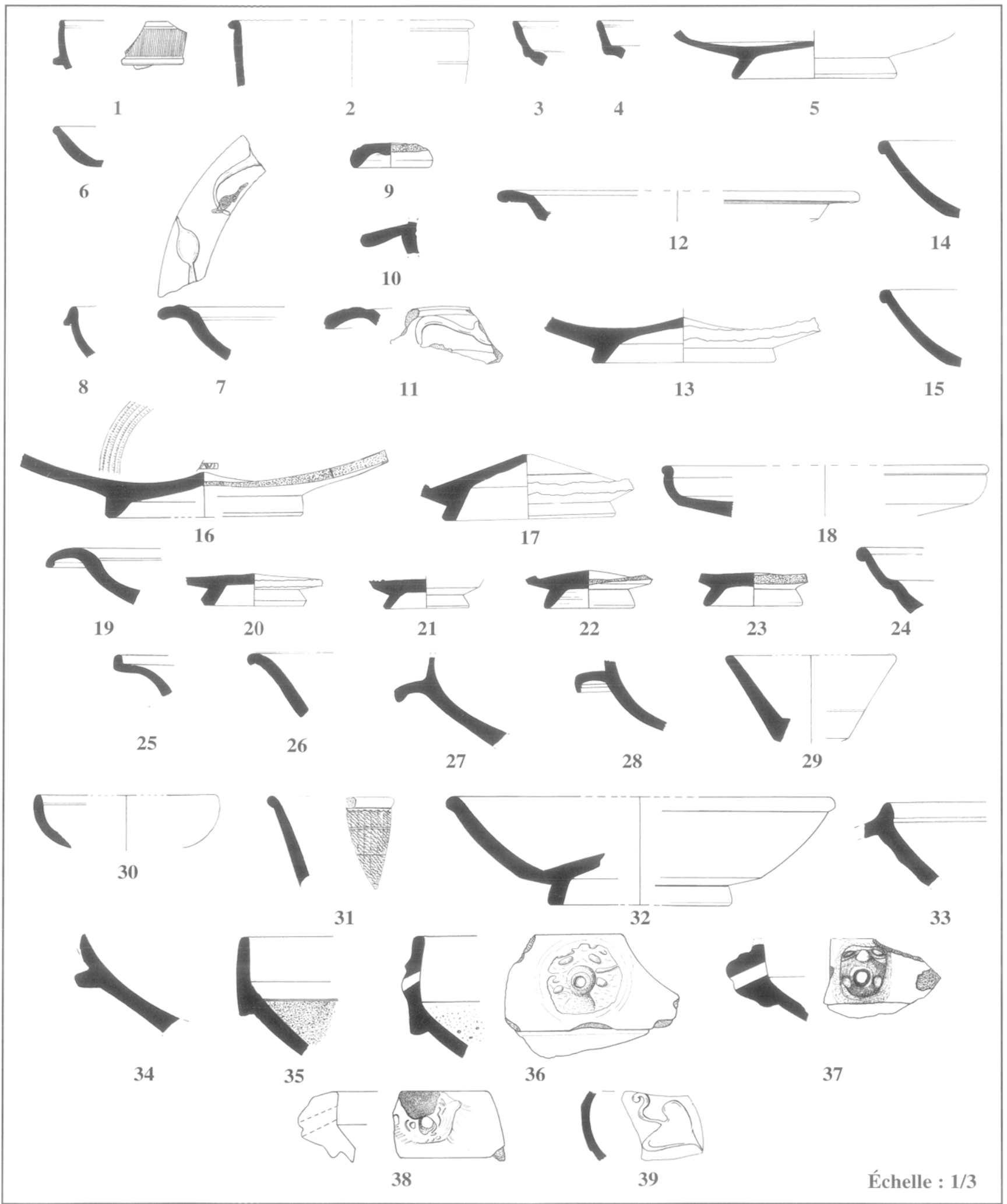




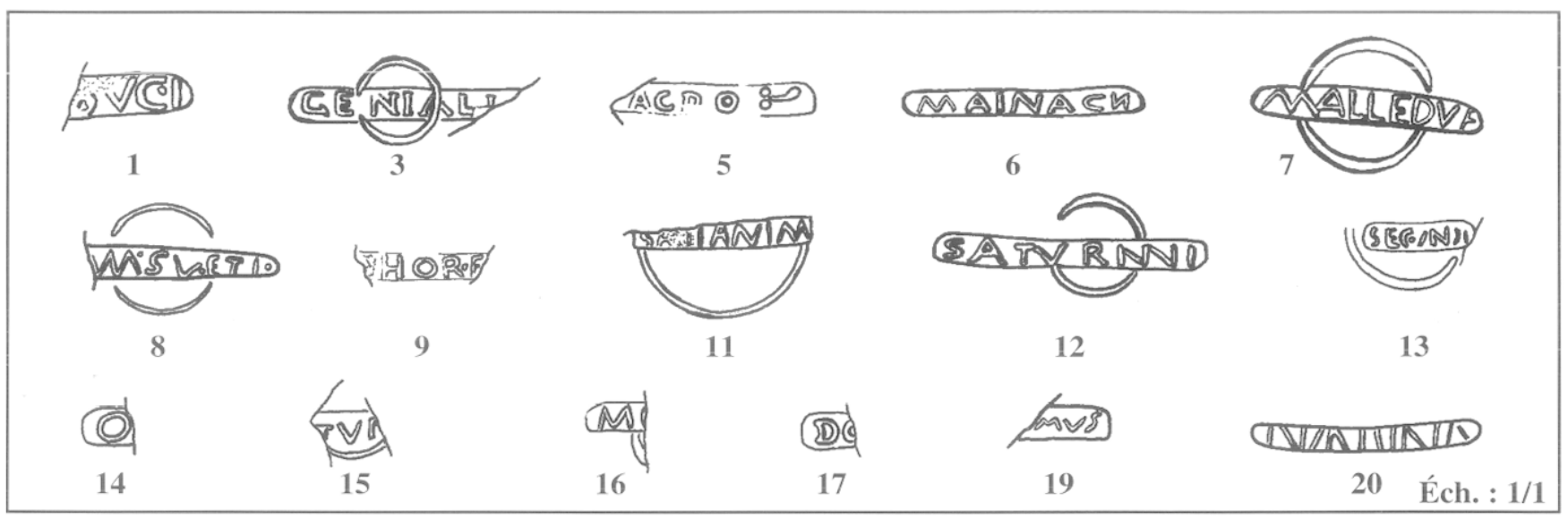




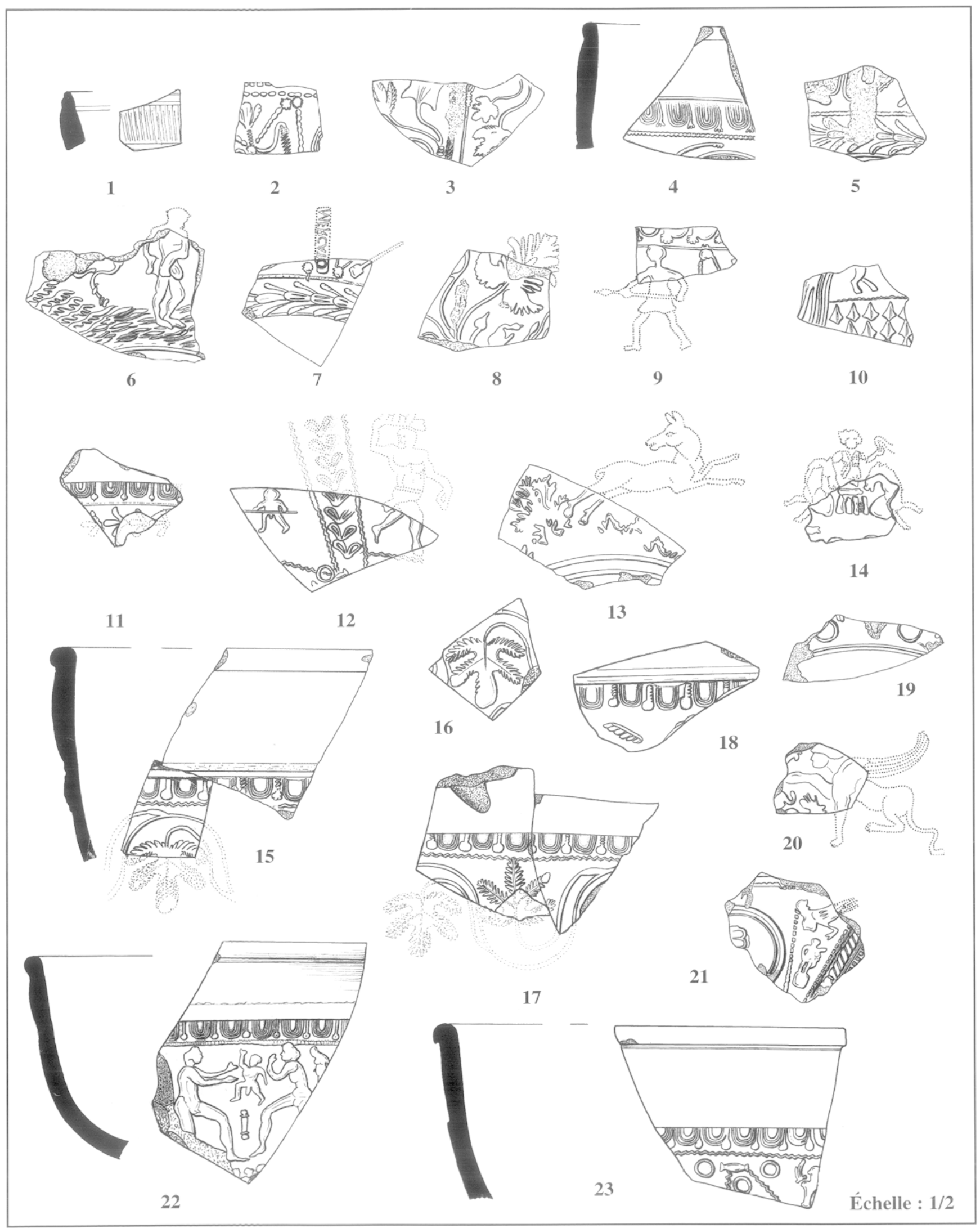




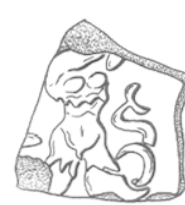

24

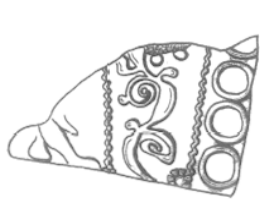

25

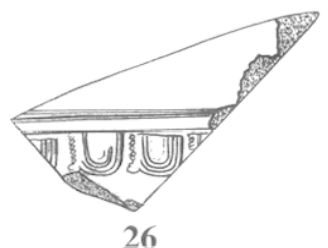

26

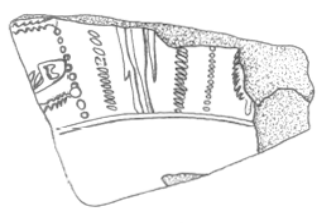

27

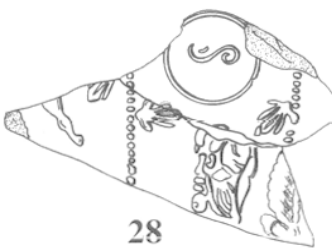

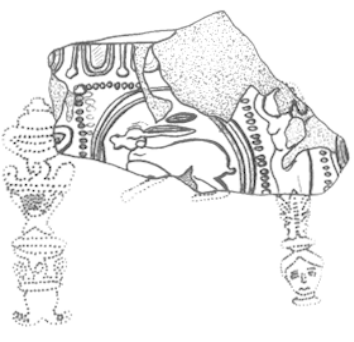

29

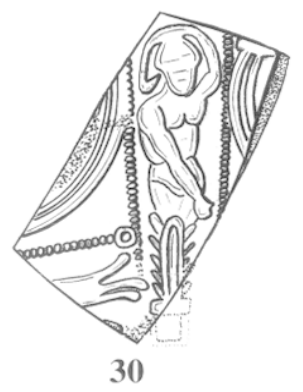

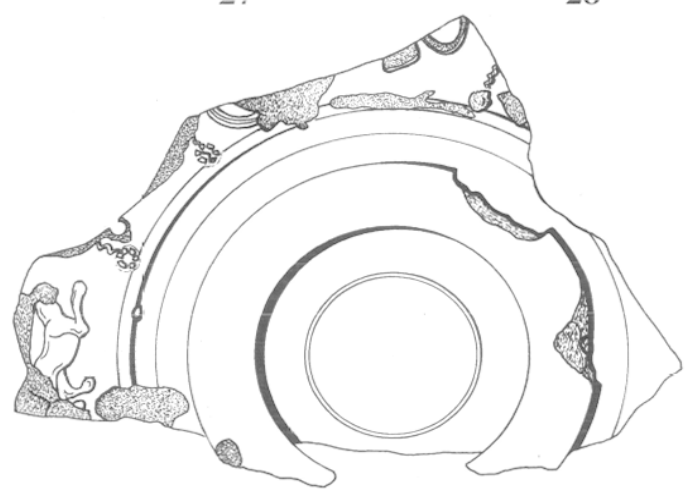

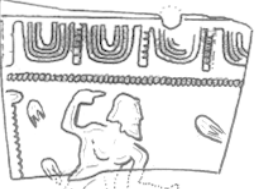

32

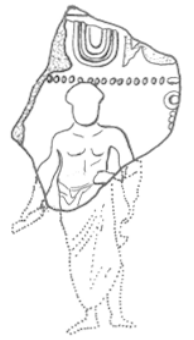

35

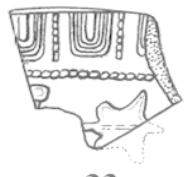

33

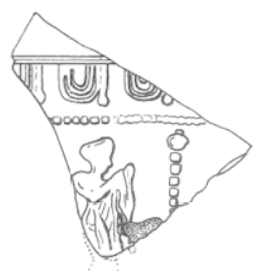

36

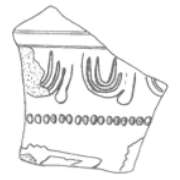

37

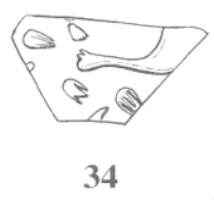

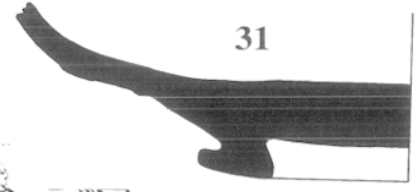
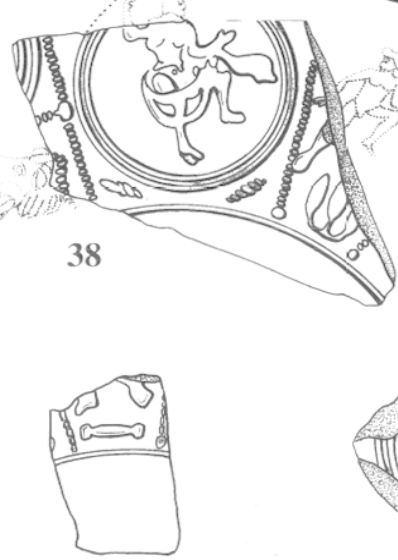

40

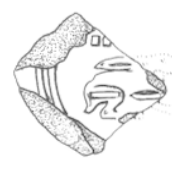

41

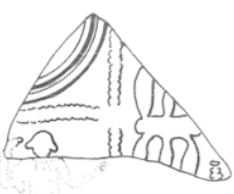

39

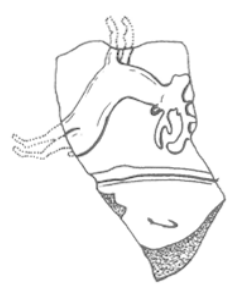

42

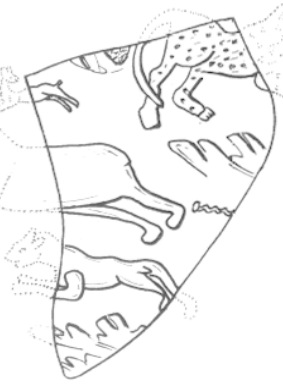

43

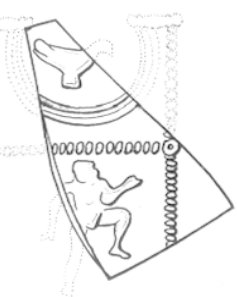

44

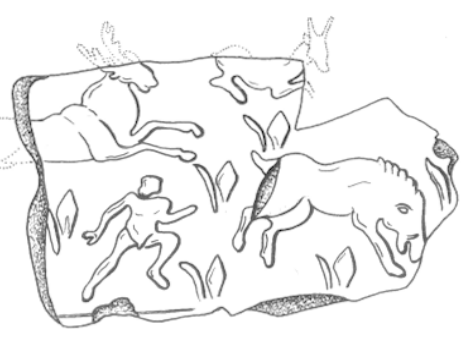

45 

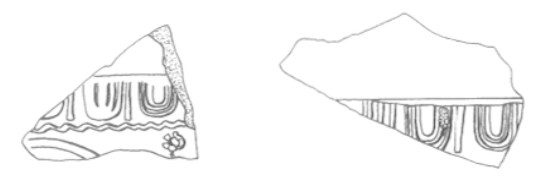

47

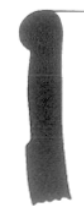

46

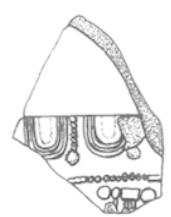

48

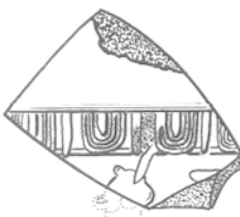

49

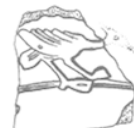

50

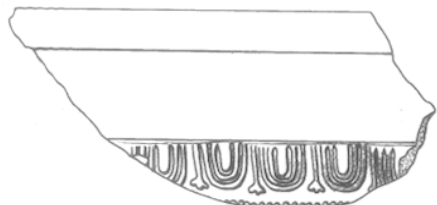

51

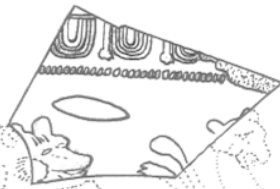

53

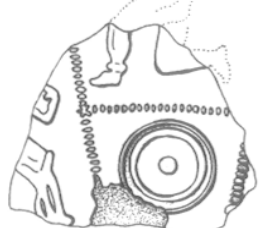

54
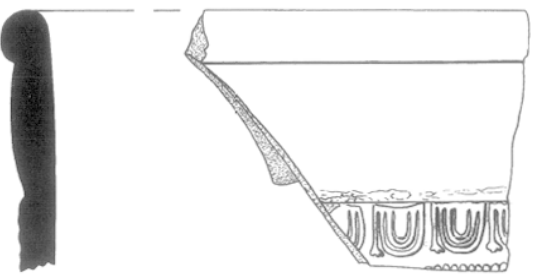

52

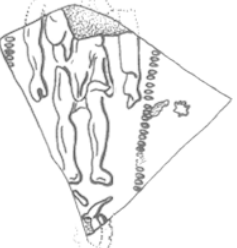

55

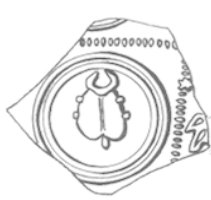

56

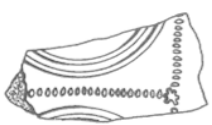

57

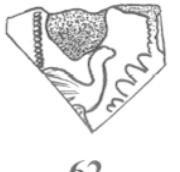

62

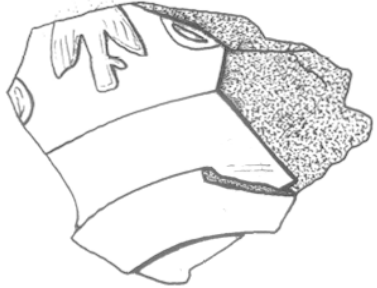

63

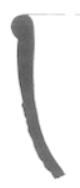

all

59

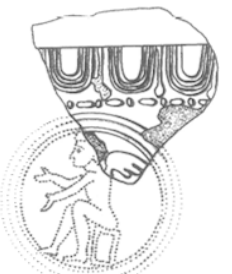

60

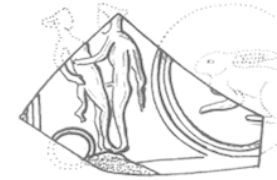

61

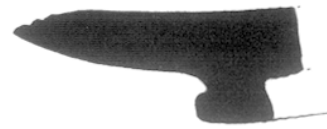

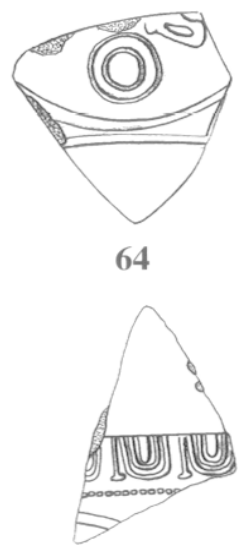

65

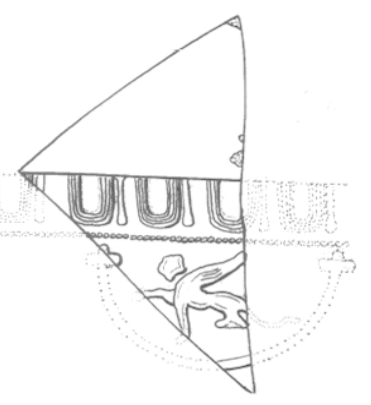

66

Échelle : $1 / 2$ 


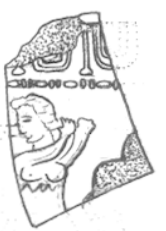

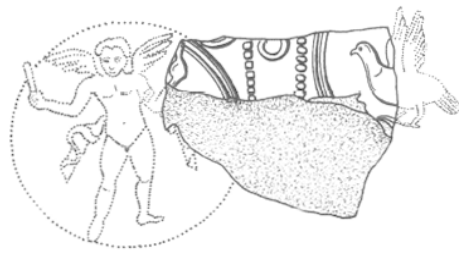

69

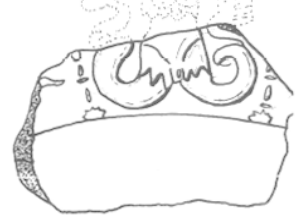

70

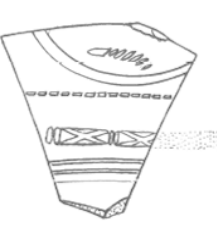

71
67

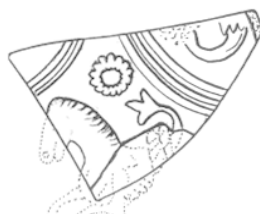

72

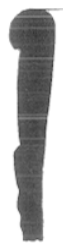

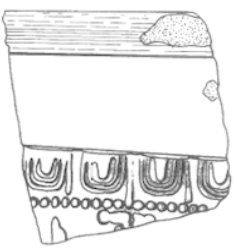

77
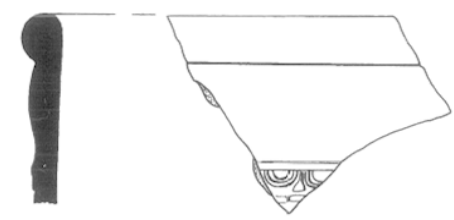

80

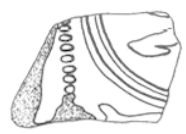

81

1

82
68

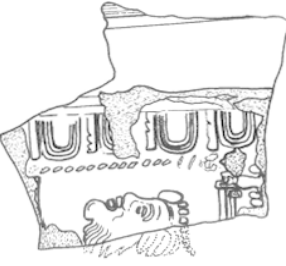

73

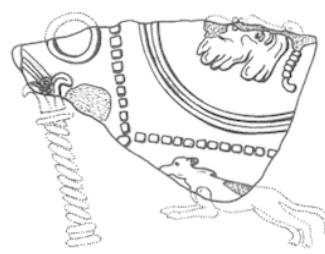

74

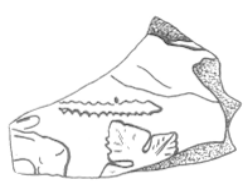

75

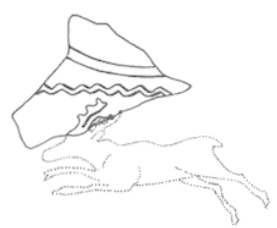

76

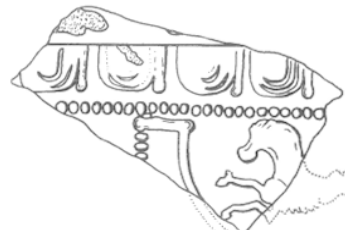

79
78

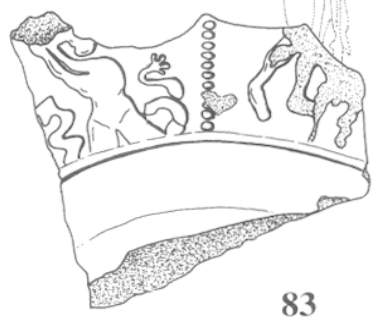

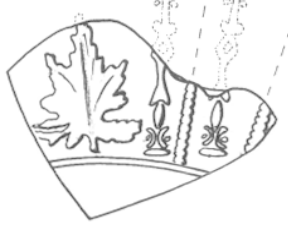

84

85

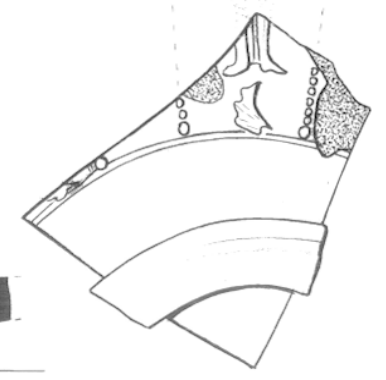

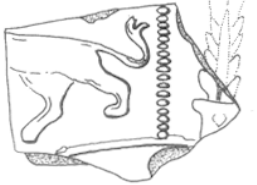

86

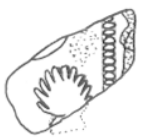

87

Échelle : 1/2 


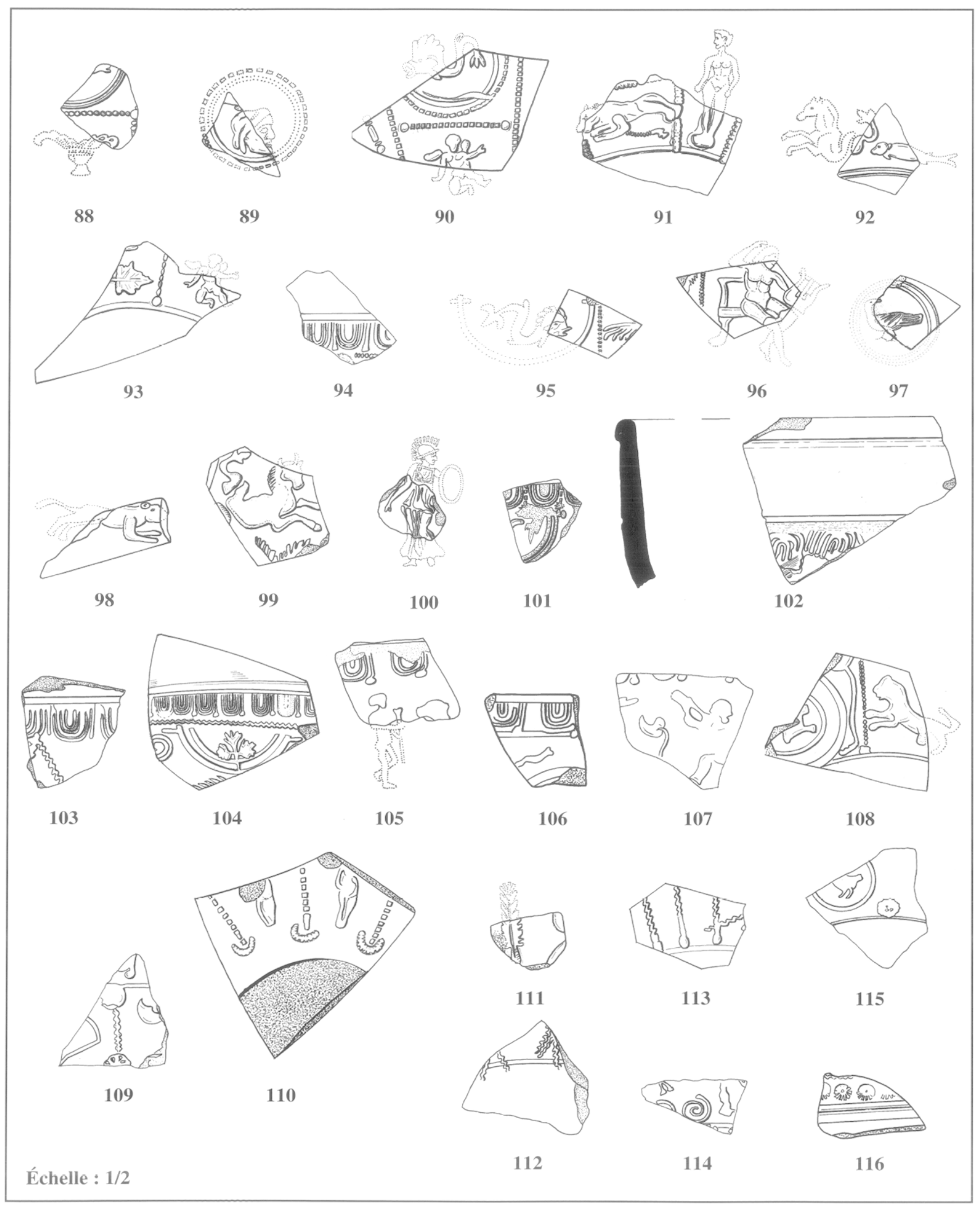




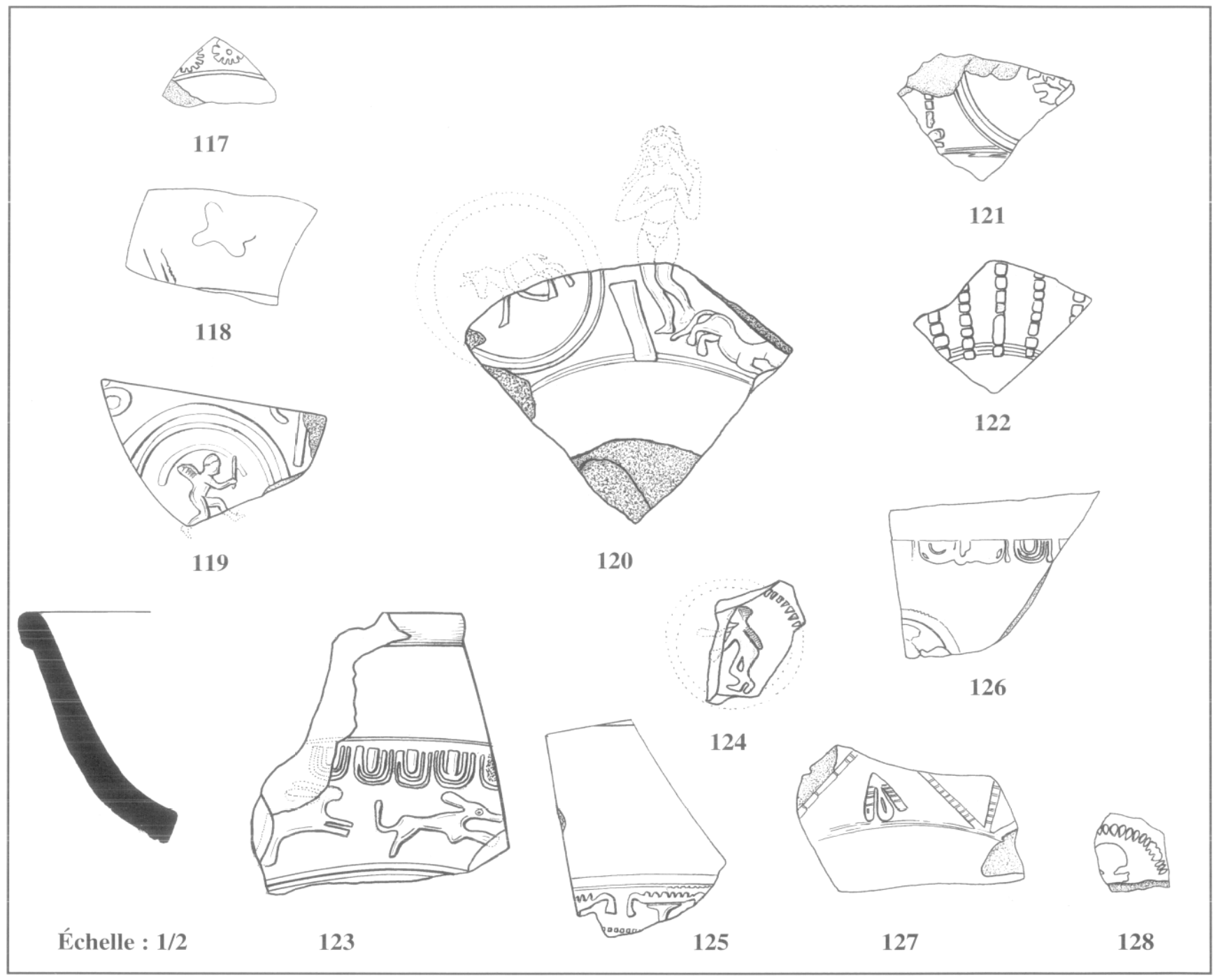




\begin{tabular}{|c|c|c|c|c|c|c|c|c|c|c|c|c|c|}
\hline & \multicolumn{3}{|c|}{$S G$} & \multicolumn{3}{|c|}{ CG } & \multicolumn{3}{|c|}{$E G$} & \multicolumn{3}{|c|}{$C / E G$} & \multirow[t]{2}{*}{ Total } \\
\hline & $B$ & $\mathrm{P}$ & $\mathrm{F}$ & $B$ & $\mathrm{P}$ & $\mathrm{F}$ & $B$ & $\mathrm{P}$ & $\mathrm{F}$ & $B$ & $\mathrm{P}$ & $\mathrm{F}$ & \\
\hline \multicolumn{14}{|l|}{ Tasses } \\
\hline Drag. $24 / 25$ & 1 & - & - & - & - & - & - & - & - & - & - & - & 1 \\
\hline Ritterling 9 & 1 & - & - & - & - & - & - & - & - & - & - & - & 1 \\
\hline O\&P;LIV;8-9 & 2 & - & - & - & - & - & - & - & - & - & - & - & 2 \\
\hline Drag. 27 & 15 & 11 & 8 & 12 & 11 & 7 & - & - & - & - & - & - & 64 \\
\hline Drag.33 & 1 & - & - & 60 & 24 & 11 & 23 & 4 & 6 & 1 & 0 & 0 & 130 \\
\hline Drag. 35 & 5 & 1 & 1 & 4 & - & - & 2 & - & - & 2 & - & - & 15 \\
\hline Drag. 40 & - & - & - & 1 & - & - & - & - & - & - & - & - & 1 \\
\hline Drag. 46 & - & - & - & 1 & - & - & - & - & - & - & - & - & 1 \\
\hline O\&P, LV, 13 & - & - & - & 2 & - & - & - & - & - & - & - & - & 2 \\
\hline Bet \& M. 47 & - & - & - & 1 & - & - & - & - & - & - & - & - & 1 \\
\hline \multicolumn{14}{|l|}{ Assiettes } \\
\hline Drag. 15/17 & 8 & 4 & - & - & - & - & - & - & - & - & - & - & 12 \\
\hline Drag. 18 & 15 & 1 & 7 & - & - & - & - & - & - & - & - & - & 23 \\
\hline $\begin{array}{l}\text { Drag. } 18 / 31 \text { ou } \\
31\end{array}$ & - & - & - & $\begin{array}{r}10 \\
7\end{array}$ & $\begin{array}{r}11 \\
5\end{array}$ & 47 & 57 & 6 & 1 & 1 & - & - & 334 \\
\hline Drag. 31 & - & - & - & 15 & 13 & 4 & 20 & 3 & 2 & - & - & - & 57 \\
\hline Drag. 32 & - & - & - & - & - & - & 1 & - & - & - & - & - & 1 \\
\hline Drag.36 & 7 & 1 & 3 & 17 & 6 & 4 & 4 & - & - & - & - & - & 42 \\
\hline Wa.79/80 & - & - & - & 2 & 2 & 1 & - & 2 & - & - & - & - & 7 \\
\hline Curle 15 & - & - & - & 3 & 2 & - & - & - & - & - & - & - & 5 \\
\hline \multicolumn{14}{|l|}{ Bols à collerette } \\
\hline Curle 21 & - & - & - & 2 & - & - & 1 & - & - & - & - & - & 3 \\
\hline Drag. 38 & - & - & - & 6 & 1 & 2 & 5 & - & - & - & - & - & 14 \\
\hline Drag. 43 & - & - & - & - & - & - & - & - & 1 & - & - & - & 1 \\
\hline Drag. 43 ou 45 & - & - & - & - & 4 & 1 & 2 & 40 & 16 & - & - & - & 63 \\
\hline Drag. 45 & - & - & - & 9 & 5 & - & 61 & 18 & 1 & - & - & - & 94 \\
\hline Drag. 44 & - & - & - & - & 2 & - & - & - & - & - & - & - & 2 \\
\hline Bols décorés & & & & & & & & & & & & & \\
\hline Drag. 29 & 1 & 1 & - & - & - & - & - & - & - & - & - & - & 2 \\
\hline Drag. 30 & - & 1 & - & - & 4 & - & - & - & - & - & - & - & 5 \\
\hline Drag. 37 & 1 & 8 & - & 52 & 95 & 9 & 18 & 18 & 9 & - & - & - & 210 \\
\hline Gobelets & - & - & - & - & 2 & - & - & - & - & - & - & - & 2 \\
\hline Indéterminé & 4 & 4 & 2 & 1 & $\begin{array}{r}16 \\
8 \\
\end{array}$ & 12 & - & 48 & - & - & - & - & 239 \\
\hline Total & & 113 & & & 847 & & & 369 & & & 4 & & 1333 \\
\hline
\end{tabular}

Tableau récapitulatif des tessons par région, par type et par forme. 